\title{
THESIS
}

\section{EVALUATING THE EFFECTIVENESS OF VARYING DOSES OF SUPPLEMENTAL TRYPTOPHAN AS A CALMATIVE IN HORSES}

\author{
Submitted by \\ Brittany P. Davis \\ Department of Animal Sciences
}

\author{
In partial fulfillment of the requirements \\ For the Degree of Master of Science \\ Colorado State University \\ Fort Collins, Colorado
}

Spring 2016

Master's Committee:

Advisor: Temple Grandin

Terry E. Engle

Jason I. Ransom

Donald C. Rojas 
Copyright by Brittany Davis 2016

All Rights Reserved 


\section{ABSTRACT \\ EVALUATING THE EFFECTIVENESS OF VARYING DOSES OF SUPPLEMENTAL TRYPTOPHAN AS A CALMATIVE IN HORSES}

Tryptophan (Trp), the amino acid precursor to serotonin, is a common ingredient in many commercial equine calming supplements. However, there is little scientific research to support the efficacy of tryptophan at modifying horse behavior. The objective of this study was to examine how various doses of tryptophan supplementation impacted reactive behavior and physiological stress measurements in the horse. Eleven horses ( 9 geldings, 2 mares) were given four treatments $-0 \mathrm{mg}$ Trp/kg bodyweight (CON), $20 \mathrm{mg}$ Trp/kg bodyweight (LOW), $40 \mathrm{mg}$ Trp $/ \mathrm{kg}$ bodyweight (MED), and $60 \mathrm{mg}$ Trp/kg bodyweight (HIGH) - in a randomized crossover design. Each treatment lasted three days. On Days 1 and 3 of each treatment, horses underwent a behavior test to measure startle response. Heart rate measurements and the speed at which the horses fled from startling stimuli were recorded. In addition, serum glucose, lactate, and cortisol levels were analyzed both immediately before the startle test and again 15 minutes after the test. Significant sedative effects were seen at LOW Day 1 on heart rate increase during the startle test $(P=0.05)$ and on change in serum lactate levels $(P=0.03)$. At MED Day 1 , sedative effects were seen on change in serum cortisol levels $(P=0.01)$. Some excitatory effects were seen at MED Day 3 on the time for heart rate to return to baseline after the startle test $(P=0.03)$. No significant effects were seen at HIGH Day 1 or Day 3. A subset of blood samples was analyzed for serum free Trp and the ratio of Trp to other large neutral amino acids, which verified treatment effect. 


\section{ACKNOWLEDGEMENTS}

There are so many people I would like to thank for their help and guidance in completing this project. I learned so much from my advisor, Dr. Temple Grandin. Working with her has been the opportunity of a lifetime. I am so grateful for the help and support of my committee members as well. Dr. Terry Engle, for advising me when I didn't know what to do, for always being willing to meet with me to answer my never-ending stream of questions, and for reassuring me that everything was going ok. Dr. Jason Ransom, for his insights, suggestions, and guidance; his commitment and willingness to help didn't waiver, even when he relocated to Washington. Dr. Don Rojas, for providing new insight on the project.

I appreciate Dr. Tanja Hess' advice and assistance with balancing the horses' diets. I would also like to thank Dr. Karen Sellins for her patience and help with ordering supplies for the project, finding volunteers, and in teaching me the laboratory procedures necessary to analyze the blood samples. I am sincerely grateful to Dr. Ann Hess, who took the time to meet with me on several occasions, and who patiently helped me work through the statistical analysis. I also appreciate Dr. Jerry Black's assistance in finding horses for me to use for the project and Wayne Miller's help with setting everything up at the Equine Teaching and Research Center and making sure we had access to what we needed throughout the project.

I am especially grateful to all the undergraduate and graduate student volunteers for all of their help in the lab and in taking care of the horses over the course of the 40-day experiment. Despite the below freezing temperatures, 5 AM start times, and incessant need for clean stalls, volunteers continued to show up. This project would not have been completed without their help. 
Last, but certainly not least, I would like to express my heartfelt thanks to my friends and family. To my mom, who was never more than a phone call away and who flew across the country in the middle of winter to help me when the project was first getting started. To my sister, for always being able to make me laugh when I needed it and whose voice reminded me what sanity was. And to Alex Harvey, who despite everything she's done, still managed to support me in more ways than she knows. This project and this degree have been harder in more ways than I imagined but I am so grateful to have met and to have had the support of so many genuine and incredible people throughout the process. I will never forget the things I learned here. 


\section{TABLE OF CONTENTS}

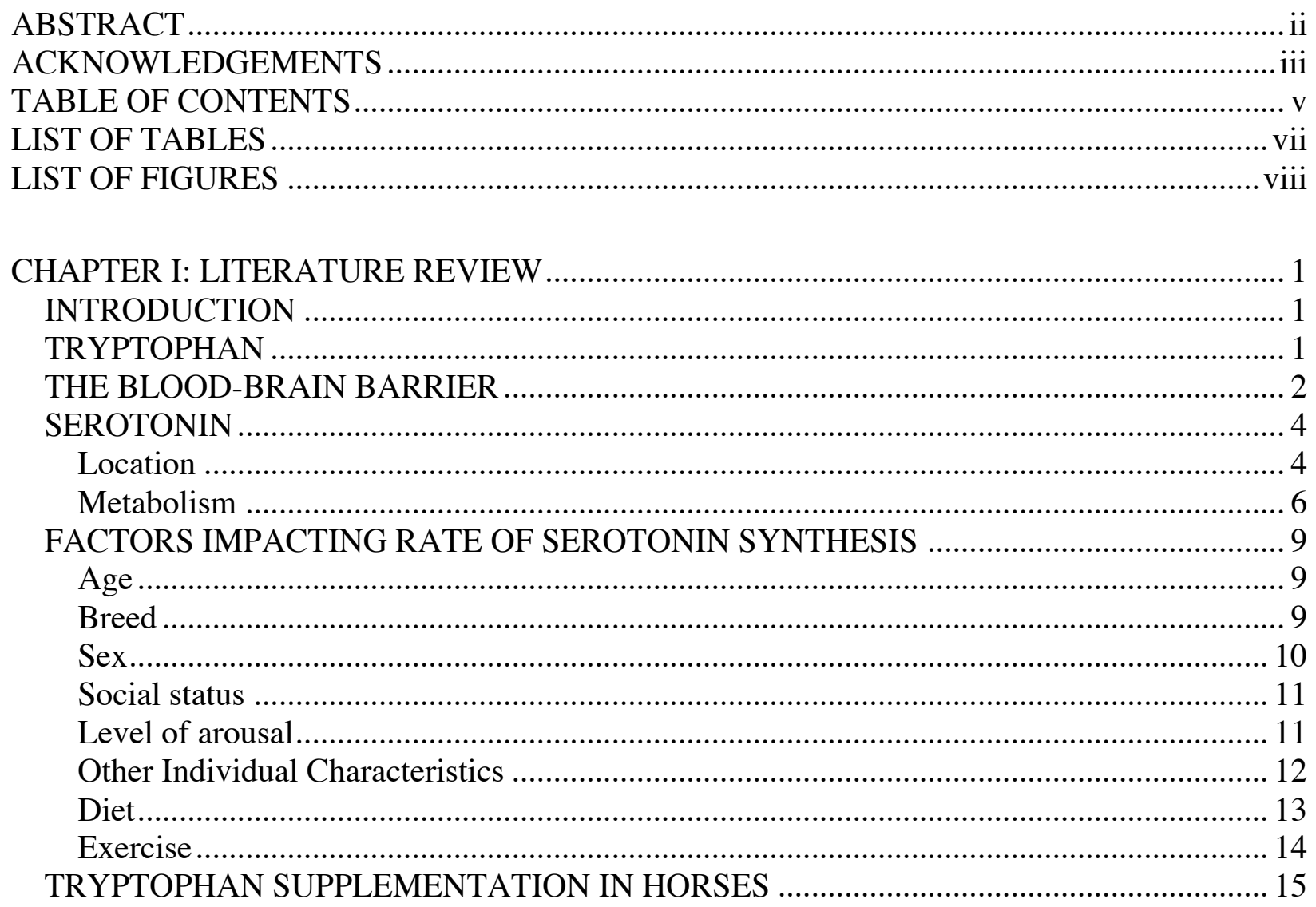

CHAPTER II: EVALUATING THE EFFECTIVENESS OF VARYING DOSES OF
SUPPLEMENTAL TRYPTOPHAN AS A CALMATIVE IN HORSES
INTRODUCTION
MATERIALS AND METHODS
Horses and Care
Study Design
Behavior Testing
Blood Sample Collection and Analysis
Statistical Analysis

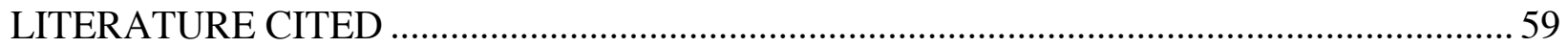




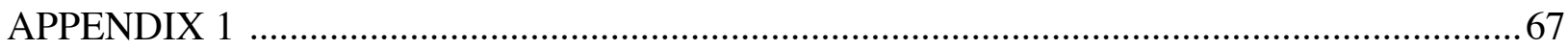

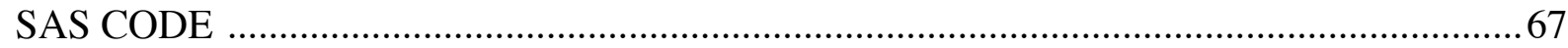

Analyze Treatment Effects on Behavioral and Physiological Response Variables on Day 1

A

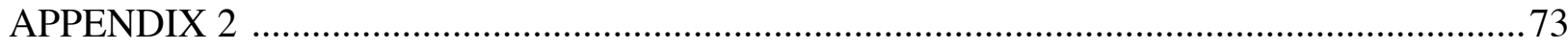

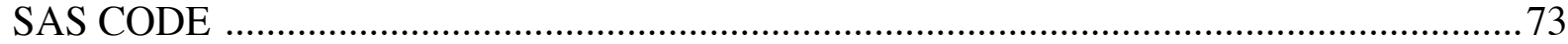

Analyze Treatment Effects on Behavioral and Physiological Response Variables on Day 3

.

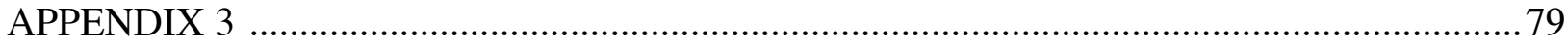

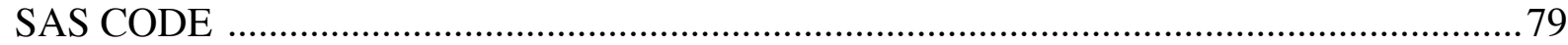

Analyze Serum Amino Acids of Group 1 Horses ..............................................................79

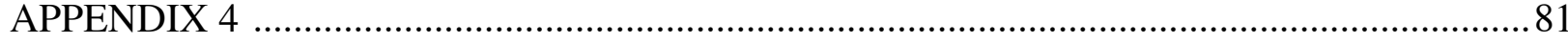

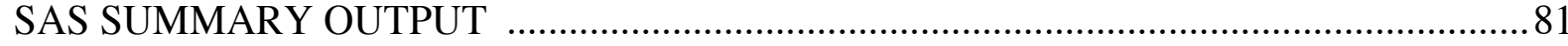

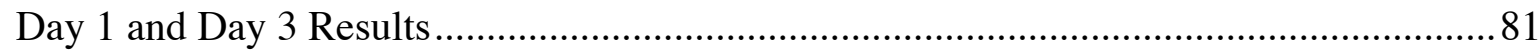

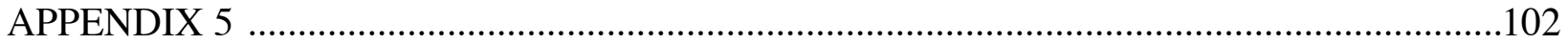

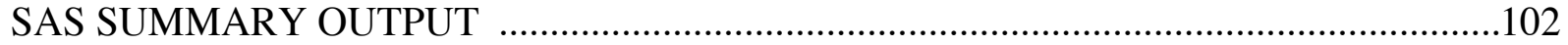

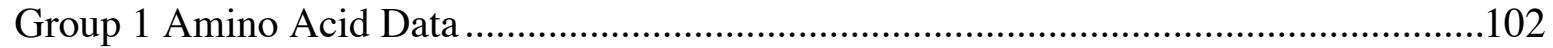




\section{LIST OF TABLES}

TABLE 1.1. Review of research supplementing tryptophan to various species

TABLE 1.2. Summary of paste supplements containing tryptophan available to horse owners in

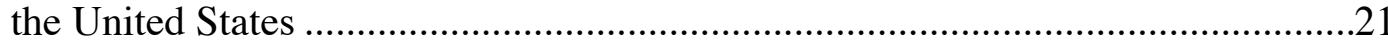

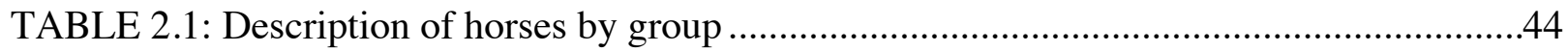

TABLE 2.2. Variance between horses ...........................................................................45

TABLE 2.3. Guaranteed analysis of feedstuffs on as-fed basis ..........................................46

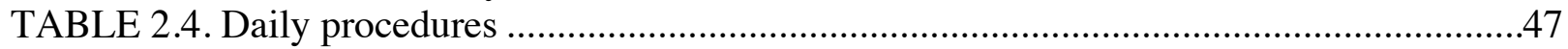

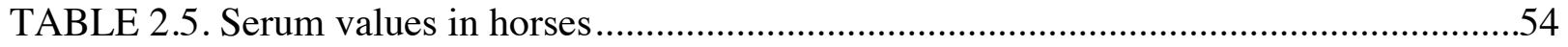

TABLE 2.6. Model covariates - correlation to response variables and influence in models ......55

TABLE 2.7. Effect of tryptophan treatment on physiological and behavioral response variables: least squares means \pm standard error ..........................................................56

TABLE 2.8. Differences of least squares means for serum free tryptophan ( $\mu \mathrm{mol} / \mathrm{L})$ Group 1

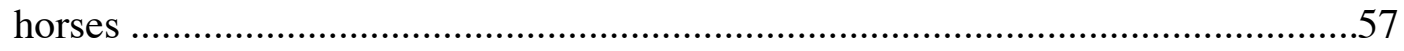

TABLE 2.9. Differences of least squares means for Trp:LNAA Group 1 horses ....................57 


\section{LIST OF FIGURES}

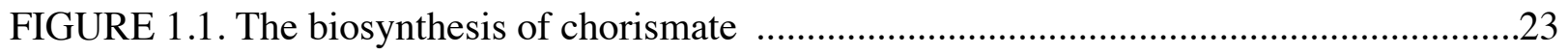

FIGURE 1.2. Pathways for the synthesis of tryptophan .................................................24

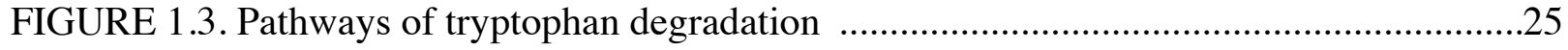

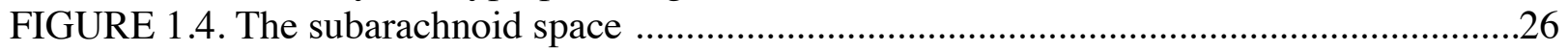

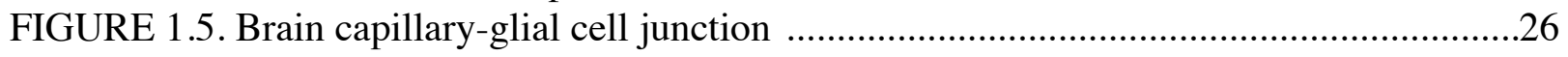

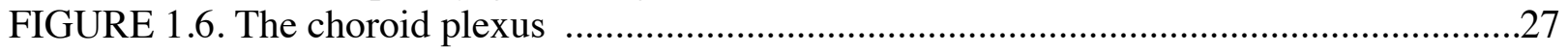

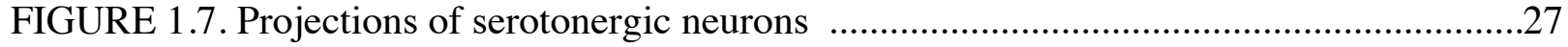

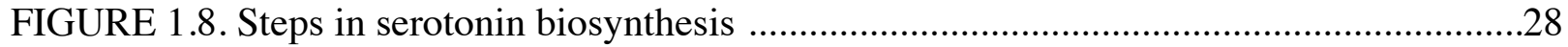

FIGURE 1.9. Exercise and diet composition impact the rate of serotonin biosynthesis ...........28

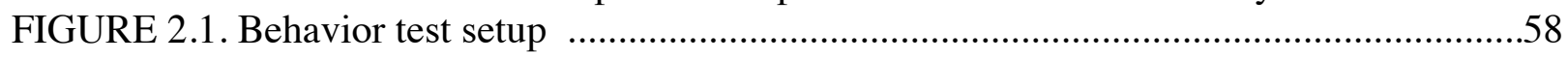

FIGURE 2.2. Differences of least squares means for serum free tryptophan $(\mu \mathrm{mol} / \mathrm{L})$ Group 1

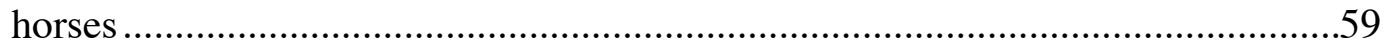

FIGURE 2.3. Differences of least squares means for Trp:LNAA Group 1 horses ..................59 


\section{CHAPTER I: LITERATURE REVIEW}

\section{INTRODUCTION}

This review will begin by covering some of the biochemical properties and pathways of tryptophan. We will discuss the physiology of the blood-brain barrier and how tryptophan is transported into the central nervous system. From here, we will delve into serotonin biosynthesis and metabolism in the brain. We'll cover some of the physiology and effects this neurotransmitter has on the body, particularly on mood. Finally, we'll review the research on supplemental tryptophan in humans and animals, focusing primarily on the horse. The purpose of this literature review is to provide a general understanding of the biochemistry, neurophysiology, and research that creates the platform which the experiment described in Chapter II is based upon.

\section{TRYPTOPHAN}

L-Tryptophan (Trp; L- $\alpha$-aminoindole-3-propionic acid) is the least abundant amino acid found in tissues and food, occurring in approximately $1.4 \%$ of protein (Fernstrom and Wurtman, 1974; Voet and Voet, 1995). Tryptophan was first isolated from casein in 1902 (Yao et al., 2011). It is a nonpolar compound with an indole group and is the largest essential amino acid, with a molar mass of $204.225 \mathrm{~g} / \mathrm{mol}$. The carboxyl group on tryptophan has a $\mathrm{pK}_{\mathrm{a}}$ value of 2.46 and the amine group has a $\mathrm{pK}_{\mathrm{a}}$ of 9.41. (Voet and Voet, 1995) Only plants and microorganisms have the enzymes necessary to synthesize tryptophan, making it one of the essential amino acids for monogastric animals and young ruminants, before weaning (Yao et al., 2011).

The reactions in tryptophan synthesis are shown in Figures 1.1 and 1.2. Aromatic amino acid synthesis begins with the same steps: phosphoenolpyruvate (a glycolytic pathway intermediate) and erythrose-4-phosphate (a pentose phosphate pathway intermediate) combine and undergo seven enzyme-catalyzed reactions. The resulting compound, chorismate, can be utilized 
to form tryptophan, tyrosine, or phenylalanine. In the biosynthesis of tryptophan, four more enzyme-catalyzed reactions take place to yield indole-3-glycerol phosphate. The last two steps require tryptophan synthase. The alpha unit of this enzyme first cleaves the compound into indole and glyceraldehyde-3-phosphate. The indole is then channeled through a tunnel in the enzyme to the beta subunit, where it is joined with serine to form tryptophan. The channeling step of tryptophan synthase is unique and important because it prevents the nonpolar indole intermediate from escaping the cell via diffusion and from being degraded (Voet and Voet, 1995).

Understanding the pathways of tryptophan degradation (shown in Figure 1.3) and their products is important in identifying how tryptophan is being metabolized and what it is being used for in the body. There are a few notable products of tryptophan degradation. Kynurenine is one such intermediate in tryptophan degradation. It is further degraded in several steps to yield alanine, which may go on to yield pyruvate. One of these steps is catalyzed by kynureninase, an enzyme dependent upon pyridoxal 5'-phosphate (PLP), the active form of vitamin B6. This is notable because, while many reactions involving amino acids are PLP-dependent, the cofactor usually cleaves different bonds than the ones broken in this reaction. PLP is also necessary in tryptophan biosynthesis and it will be an important cofactor in reactions to be discussed later. Quinolinate, which is an $\mathrm{NAD}^{+}$and $\mathrm{NADP}^{+}$precursor in the liver and kidneys, is another tryptophan metabolite. An alternative degradation pathway concludes with reactions identical to those seen in the breakdown of lysine; acetoacetate is the final metabolite (Voet and Voet, 1995).

\section{THE BLOOD-BRAIN BARRIER}

While water, carbon dioxide, oxygen, and lipid-soluble compounds pass through plasma membranes and equate between the blood and brain easily, the blood-brain barrier is almost impermeable to larger compounds like plasma proteins. However, there are some areas of the 
brain - parts of the hypothalamus, pineal gland, and medulla-where compounds diffuse more easily (Guyton and Hall, 2000). Sites of the blood-brain barrier include the arachnoid membrane, the blood vessels in the subarachnoid space, and the choroid plexus. The arachnoid villus is shown in Figure 1.4. When pressure of the cerebrospinal fluid exceeds that of the sinus blood, the arachnoid villi will open, allowing the bulk movement of metabolic and waste molecules from the CSF into venous sinus blood (Rapoport, 1976). Unlike other capillaries in the body, those found in the brain are connected by tight junctions and, as shown in Figure 1.5, glial cells cover about $85 \%$ of the vessel surface (Rapoport, 1976). This serves as a second site of the blood-brain barrier. The choroid plexus is the third site of the blood-brain barrier and is shown in Figure 1.6. The choroid plexus secretes cerebrospinal fluid and is located in the lateral, third, and fourth ventricles (Rapoport, 1976).

Tryptophan, like some other amino acids, binds non-covalently to serum albumin (Yao et al., 2011). Actually, most (80-90\%) of circulating tryptophan is bound to albumin (Bosch et al., 2007). Several studies provide evidence that increasing free tryptophan relates to a greater ability of the amino acid to pass the blood brain barrier (Bosch et al., 2007; Davis et al., 2000; Farris et al., 1998; Fernstrom and Wurtman, 1972b; Grimmett and Sillence, 2005). Free tryptophan competes with other large neutral amino acids (LNAA) - including tyrosine, phenylalanine, leucine, isoleucine, and valine-for the same transporter to cross the blood-brain barrier (Fernstrom, 2013). This facilitated transporter is called the L-system. It has little dependence upon $\mathrm{pH}$ and no sodium dependence. LNAA, including tryptophan, are transported down their concentration gradients via the L-system (Rapoport, 1976). The concentrations of the amino acids found in plasma are normally much lower than the $K_{m}$ values and saturation capacity of the transport system (Rapoport, 1976). The facilitated diffusion of tryptophan into the brain is a linear 
function of concentration up to many times plasma levels (Rapoport, 1976). Once inside the brain, tryptophan can be used to synthesize serotonin, a biogenic amine with many functions, some of which will be discussed in the following sections.

"An excessive plasma concentration of one of a competing set of amino acids reduces brain uptake of others of the set, thereby modifying synthesis of protein, myelin, and neurotransmitters, and altering cellular respiration and replication" (Rapoport, 1976). A lot of what is known about competition between amino acids for entry into the brain is known because of aminoacidurias, diseases in which an individual lacks critical enzymes necessary in amino acid metabolism. Phenylketonuria, for example, occurs in people with a deficiency of phenylalanine hydroxylase, an enzyme necessary to convert phenylalanine to tyrosine. As a result, phenylalanine and its metabolic byproducts build up in the blood and have neurotoxic effects. All babies born in the United States are now tested for phenylketonuria at birth; if treated with a diet low in phenylalanine and high in the essential amino acids that compete with phenylalanine, detrimental effects such as mental retardation can be prevented. Understanding the etiology of this disease provided researchers with an understanding of the competitive inhibition between amino acids with similar structural and chemical properties for transport across the blood-brain barrier. High blood phenylalanine impairs serotonin synthesis by interfering with tryptophan transport into the brain. Serum serotonin is decreased in phenylketonuric patients and brain serotonin is decreased in phenylalanine-loaded animals. (Rapoport, 1976)

\section{SEROTONIN}

\section{Location}

Serotonin is an indoleamine found throughout the body and functions in several different systems. Most (80-95\%) of serotonin in the body is located within the gastrointestinal tract, 
specifically in enterochromaffin cells and in enteric neurons (Kim and Camilleri, 2000). Here, serotonin plays a crucial role in gut secretion, motility, and sensation (Yao et al., 2011). Serotonin is also an important constituent of platelets and contributes to blood coagulation (Kim and Camilleri, 2000). Although serotonin is a hormonally active substance in the blood, it does not have a direct effect on the brain because of the blood-brain barrier (Voet and Voet, 1995). Instead, precursors are transported across the barrier and serotonin is synthesized in the central nervous system, where it influences cognition, sleep, mood, and appetite. Serotonin is also a factor in some neurological conditions including depression, anxiety disorders, schizophrenia, and eating disorders (Yao et al., 2011). Scientists credit the multiple roles of serotonin as a hormone and a neurotransmitter to evolutionary opportunism rather than to related physiological significance (Voet and Voet, 1995).

The cell bodies of serotonergic neurons are located in the dorsal raphe nucleus of the midbrain. However, these neurons have projections extending to many regions of the brain and have different influences on mood and cognition, shown in Figure 1.7.

There are at least thirteen different $G$ protein-coupled receptors that mediate serotonin activity as a neurotransmitter (Hannon and Hoyer, 2008). Some receptors are specific to certain areas of the brain and on the serotonergic neurons themselves. Based on structure, transduction properties, and mode of operation, these receptors are divided into seven types (5-HT $\mathrm{H}_{1}$ through 5$\mathrm{HT}_{7}$ ) (Hannon and Hoyer, 2008). Corr (2006) describes these receptors in more detail: the two main presynaptic receptors are 5- $\mathrm{HT}_{1 \mathrm{~A}}$, which slows neuronal firing, and 5- $\mathrm{HT}_{1 \mathrm{D}}$, which detects 5$\mathrm{HT}$ in the synapse. When 5-HT $\mathrm{HD}_{1 \mathrm{D}}$ is occupied, release of 5-HT is inhibited. Postsynaptic receptors include 5- $\mathrm{HT}_{1 \mathrm{~A}}, 5-\mathrm{HT}_{1 \mathrm{D}}, 5-\mathrm{HT}_{2 \mathrm{~A}}, 5-\mathrm{HT}_{2 \mathrm{C}}, 5-\mathrm{HT}_{3}$, and 4- $\mathrm{HT}_{4}$ (Corr, 2006). Serotonergic neurons also contain norepinephrine receptors that modulate 5-HT release (Corr, 2006). In addition, 
serotonergic neurons in the dorsal raphe nucleus can be inhibited by GABAergic interneurons from the prefrontal cortex (Robbins, 2005). These examples demonstrate the interconnectedness of neural pathways. Although a lot of research in psychology and pharmacology is devoted to further understanding the complex functions and mechanisms of modification of serotonin receptors, drug effects are oftentimes non-specific and influence more than one transmitter system (Mench and Shea-Moore, 1995). Hannon and Hoyer provide a more detailed review of 5-HT receptors (2008).

\section{Metabolism}

Once tryptophan crosses the blood-brain barrier, brain serotonin biosynthesis occurs in a two-step process, shown in Figure 1.8. First, tryptophan is hydroxylated into 5-hydroxytryptophan (5-HTP) by tryptophan hydroxylase. This is the rate-limiting step. Oxygen and tetrahydrobiopterin also form dihydrobiopterin in this first step. Next, 5-HTP decarboxylase, a PLP-dependent enzyme, cleaves the carboxyl group from 5-HTP to produce 5-hydroxytryptamine (5-HT), also known as serotonin (Voet and Voet, 1995). Serotonin biosynthesis rates are about 20 times higher in neuron cell bodies than in terminals (Boadle-Biber, 1993).

In the central nervous system, serotonin is inactivated primarily by reuptake by serotonergic neurons. Highly selective sodium- and chloride-dependent membrane transporters recycle the neurotransmitter back to terminal buttons (Corr, 2006). A lot of research has been dedicated to understanding the serotonin transporter gene (5-HTT), which is involved in serotonin transmission, early brain development, adult neurogenesis, and plasticity (Corr, 2006). Serotonin can also be destroyed by monoamine oxidase and aldehyde dehydrogenase, yielding 5hydroxyindole acetic acid (5-HIAA) (Kim and Camilleri, 2000). Melatonin is another potential metabolite of serotonin. Melatonin is a hormone primarily produced in the pineal gland but some biosynthesis also occurs in the retina and gastrointestinal tract (Esteban et al., 2004). Melatonin 
synthesis increases in the evening and is critical in the maintenance of the circadian clock (Piccione et al., 2005).

Because the hydroxylation of tryptophan is the rate-limiting step in serotonin biosynthesis, an increase in brain tryptophan can potentially double serotonin synthesis (Bosch et al., 2007). Esteban and others (2004) showed that, under normal conditions, the rate-limiting enzyme tryptophan hydroxylase is far from being saturated by its substrate. The availability of cofactors such as magnesium, vitamin $\mathrm{B}_{3}$, and vitamin $\mathrm{B}_{6}$ may also play a role in the hydroxylation of tryptophan (Alberghina et al., 2010b).

Two-thirds of the tryptophan available for serotonin biosynthesis comes from intracellular degradation. Diet serves as the secondary source but only 1-2\% of dietary tryptophan is converted to serotonin (Yao et al., 2011). While these proportions may seem low, the rate of serotonin synthesis has displayed more sensitivity to its dietary precursor than any other any other neurotransmitter (Fernstrom, 2013). Further evidence suggests that the amount of tryptophan available to the brain may influence serotonin biosynthesis. Studies have shown an increase in the main metabolite of serotonin, 5-hydroxyindoleacetic acid (5-HIAA), as a result of large doses of tryptophan (Fernstrom and Wurtman, 1974). Increasing dietary tryptophan stimulates an increase in brain serotonin synthesis in several species (Adeola and Ball, 1992; Laycock and Ball, 1990; Leathwood, 1987; Shea et al., 1990). Conversely, tryptophan depletion can be achieved by providing a tryptophan-free diet. Because tryptophan depletion creates a significant reduction in brain serotonin synthesis and release, it is a valuable tool used to study the brain serotonergic system and pharmacology effecting that system (Bell et al., 2001; Kantak et al., 1980).

In human medicine, drugs that increase serotonergic activity are used to treat a range of psychological and neurological conditions. These compounds act on various serotonin receptors 
to stimulate more neurotransmitter release and/or to inhibit reuptake of the neurotransmitter from the synaptic cleft. Other drugs inhibit monoamine oxidase activity, reducing the rate of serotonin degeneration. Additional treatments directly or indirectly effect dopamine, norepinephrine, and GABA pathways.

The serotonin syndrome is a consequence of excess serotonergic agonism in the central nervous system and has been seen across species, including humans, monkeys, rabbits, mice, and rats (Boyer and Shannon, 2005; Gillman, 1999). Serotonin syndrome has been associated with the use of MAOIs, tricyclic antidepressants, SSRIs, opiate analgesics, anti-migraine drugs, herbal products, and other drugs (Boyer and Shannon, 2005). The severity of the condition ranges from barely perceptible to lethal and presents a spectrum of clinical findings in people. Symptoms include: tremor, hyperreflexia, spontaneous muscle spasms, muscle rigidity, hyperthermia, agitation, sweating, shivering, diarrhea, incoordination, and delirium (Boyer and Shannon, 2005). The combination of serotonergic drugs has induced the rapid onset (minutes to hours) and progression of serotonin syndrome (Gillman, 1999). A single dose of an SSRI has also been observed to cause serotonin syndrome (Boyer and Shannon, 2005).

In the past, serotonin syndrome has been misdiagnosed as neuroleptic malignant syndrome, which presents with many of the same symptoms as serotonin syndrome but is caused by dopamine antagonists instead of serotonergic drugs (Sternbach, 1991). There are only a few distinguishing symptoms between the two conditions; namely that patients presenting with serotonin syndrome typically have hyperactive gut sounds and dilated pupils (Boyer and Shannon, 2005). Nonetheless, patient medication history is extremely important in accurate diagnosis and treatment of serotonin syndrome. In mild to moderate cases, supportive care and cessation of proserotonergic agents is effective at alleviating symptoms associated with the syndrome (Boyer and Shannon, 2005). 
Sedation, paralysis, intubation, and treatment with $5-\mathrm{HT}_{2}$ blockers may be necessary in lifethreatening cases (Boyer and Shannon, 2005; Gillman, 1999). Many cases of serotonin syndrome can be resolved within 24 hours; however, symptoms may persist in patients taking longer acting drugs (Boyer and Shannon, 2005).

\section{FACTORS IMPACTING RATE OF SEROTONIN SYNTHESIS}

The rate of brain serotonin synthesis depends on a number of things, including age, sex, breed, social status, level of arousal, and other individual characteristics. External factors such as diet and exercise can also impact the rate of serotonin synthesis.

Age

Some researches have reported that the permeability of the blood-brain barrier decreases with age (Grimmett and Sillence, 2005). In accordance with this line of thought, higher plasma tryptophan and serotonin levels are seen in foals than in adult horses (Ferlazzo et al., 2012). However, one study showed a positive correlation between age and plasma tryptophan throughout the first year in a foal's life (Alberghina et al., 2014). These data suggest that throughout a horse's lifetime, the permeability of the blood-brain barrier stops increasing, stabilizes, and eventually decreases. Farabollini et al. (1988) found that neonatal rats given serotonin antagonists were less anxious and responded more to environmental and social cues as adults. However, brain serotonin levels and turnover are similar in control and animals treated neonatally, suggesting that early effects may change receptor sensitivity (Farabollini et al., 1988).

Breed

While there is some evidence supporting the effect of breed and genetics on tryptophan availability/serotonin biosynthesis, their mechanisms remain unclear. One study noted that Arabian-type horses have higher plasma tryptophan than Anglo-Arabians (Alberghina et al., 
2014). In another study, Bagshaw et al. (1994) found that resting serum serotonin is lower in Arabian mares than in Standardbred mares. However, serum tryptophan concentrations were not significantly different between the two breeds. A separate group of researchers saw lower blood serotonin in Arabian mares compared to Swedish Warmblood mares, even though all horses were fed the same diets and housed in the same conditions (as cited in Grimmett and Sillence, 2005). It is unclear whether breed differences relate to differing absorption, transport, metabolism, and/or excretion of tryptophan. Momozawa and others (2006) investigated polymorphisms in the equine serotonin transporter gene, which controls serotonin reuptake from the synaptic gap. However, of the haplotypes identified, none were associated with anxiety scores determined through caretaker questionnaire.

$\operatorname{Sex}$

Research evaluating gender differences in serotonin biosynthesis in the human brain report conflicting results (as cited in Kim and Camilleri, 2000). However, research in animals tends to suggest that females are more sensitive to dietary changes in tryptophan than males. Dickson and Curzon (1986) reported that female rats that were fed tryptophan were more likely to exhibit side effects liked to serotonin syndrome than their male counterparts. Rouvinen et al. (1999) supplemented silver foxes with tryptophan over the course of several months. While females showed reduced fear and increased exploratory behavior, the same response was not seen in males. Henry and others found that female swine have less hypothalamic serotonin and are more sensitive to dietary changes in amino acid ratio than males (Henry et al., 1992; Henry et al., 1996). Not only do female and male (neutered and intact) pigs have different concentrations of serotonin and 5-HT metabolites in the brain, but the areas that seem to have the most serotonergic activity vary (Henry 
et al., 1996). These studies provide evidence that androgens inhibit serotonergic function in male rats and boars.

Other researchers have noted that female rats are more vulnerable than males in models of depression and show less sensitivity to serotonin receptor agonists (Kennett et al., 1986). In addition, female rats seem less sensitive than males to neonatal manipulation with serotonin receptor agonists or antagonists; possibly because of interaction between the developing serotonin system and testosterone (Albonetti et al., 1994).

\section{Social status}

Some studies show that subordinate animals have higher levels of serotonin, yet may be less sensitive to tryptophan supplementation than their dominant counterparts (Mench and SheaMoore, 1995; Raleigh, 1987). A different study showed that dominant males have twice as much blood serotonin than submissive males (Mench and Shea-Moore, 1995). These researchers hypothesized that the dominant animals were metabolizing more dietary tryptophan to serotonin peripherally instead of in the central nervous system.

\section{Level of arousal}

Several researchers have provided evidence that serotonin synthesis and precursor sensitivity are greater at higher states of arousal. This is probably because more neurotransmitter is released when the neurons fire more frequently (Young, 1991). A study by Trulson (1979) provides support for this hypothesis, showing dorsal raphe activity in cats increases with state of arousal. In addition, Chamberlain et al. (1987) concluded that altered tryptophan levels in the diet influence aggression in vervet monkeys more reliably at higher levels of arousal. Firing of action potentials may enhance tryptophan uptake into 5-HT neurons via a carrier specific to these neurons and/or tryptophan hydroxylase activity may be enhanced as a result of neuronal firing. In an in 
vitro experiment, an increase in serotonin synthesis was only observed in the presence of electrical field stimulation, even though available tryptophan was the same in stimulated and unstimulated tissues. However, in vivo studies consistently show that administration of exogenous tryptophan enhances 5-HT formation; this may be reflective of the fact that 5-HT neurons fire tonically in vivo (as cited in Boadle-Biber, 1993). Additionally, Schaechter and Wurtman (1990) provide evidence that elevating tryptophan levels in the rat hypothalamus proportionally increases 5-HT levels and release; these data support the hypothesis that serotonin release is proportionate to intracellular serotonin levels.

\section{Other Individual Characteristics}

There is some evidence in the literature that tryptophan supplementation is most effective in individuals who exhibit dysregulation of behaviors that may be under control of the serotonergic system. For example, Bell and others (2001) performed a study in humans which showed that healthy individuals experienced some mood lowering, memory impairment, and an increase in aggression due to tryptophan depletion. However, patients who had been treated for depression or panic disorder and responded well to antidepressants (particularly serotonergic agents), experienced a relapse. Interestingly, patients with untreated depression experienced no worsening in mood symptoms. These findings suggest that individual serotonergic systems may vary in sensitivity even when factors such as age and sex are held constant. Further support is provided Weld and others (1998), who saw changes in serotonin turnover (evidenced by concentrations of 5-HIAA in cerebral spinal fluid) as a result of tryptophan supplementation only in animals who displayed self-injurious behavior. 


\section{Diet}

We have already discussed how the amount of tryptophan in the diet has an impact on serotonin biosynthesis. As shown in Figure 1.9, the profile of other nutrients in the diet can influence the amount free tryptophan available for transport transport into the central nervous system and for serotonin synthesis.

The release of insulin triggers the uptake of free amino acids into peripheral tissues such as muscle (not the brain). However, because most tryptophan is bound to albumin, the rate of absorption into peripheral tissues is slower. As a result, the ratio Trp:LNAA increases, favoring tryptophan uptake by the brain and, furthermore, serotonin synthesis (Bellisle et al., 1998). Fernstrom and Wurtman (1972b) conducted a study in which insulin was administered to fasted rats. Surprisingly, this increased total plasma tryptophan up to $40 \%$ while also decreasing the concentration of other LNAA in the plasma. Two hours after receiving the insulin, brain tryptophan levels were elevated by $36 \%$ and brain serotonin levels were elevated by $28 \%$. Research by Noble and others (2007) provides further supporting evidence in the role of insulin in serotonin biosynthesis by showing that plasma tryptophan follows the glycemic response after meal-feeding Thoroughbred horses. Additionally, the Trp:LNAA ratio peaked after horses were fed a "starch and sugar" meal but stayed relatively constant in horses fed a "fat and fiber" meal (Wilson et al., 2007). There is some discrepancy in the literature, however; Alberghina and others (2010b) found that horses kept on a high fiber diet had higher plasma serotonin and tryptophan levels than horses fed a high starch diet. The conflicting results of this study may be because blood samples were taken three and six hours after feeding — at least an hour after the spikes in insulin, serotonin, and tryptophan were reported in previous studies. Also, Kim and Camilleri (2000) claim that plasma measurements of serotonin are often inaccurate, since serotonin is easily released with the agitation 
and lysis of platelets. Wurtman (2011) draws the association that "carbohydrate cravers" may be unknowingly attempting to increase serotonin synthesis using this mechanism in order to compensate for disorders linked with low serotonin.

High fat diets (or lipolysis) may also increase the amount of free tryptophan available to the central nervous system, as non-esterified fatty acids displace tryptophan from its binding site on albumin (Bosch et al., 2007). High protein diets increase plasma tryptophan levels but, because of the corresponding increase in competing LNAA, brain tryptophan and serotonin do not increase. Removing the competing amino acids from the diet has been shown to increase tryptophan and serotonin (Fernstrom and Wurtman, 1972a).

\section{Exercise}

There is evidence that exercise increases free tryptophan, the tryptophan to LNAA ratio, and brain serotonin (as cited in Bruschetta et al., 2013; Farris et al., 1998). Exercise may increase free Trp:LNAA by releasing free fatty acids, which displace tryptophan from its binding site on albumin (Grimmett and Sillence, 2005). Additionally, multiple studies have looked at the effects of tryptophan and/or serotonin in central fatigue during exercise but the results are conflicting and range from a negative correlation between serotonin and exercise endurance to the absence of an effect between tryptophan supplementation and time until exercise fatigue (Alberghina et al., 2010a; Bruschetta et al., 2013; Farris et al., 1998; Piccione et al., 2005; Vervuert et al., 2005). Some researchers believe that exercise releases peripheral serotonin from platelets, which is transported in the plasma to neurons or vascular endothelial cells (Alberghina et al., 2010a; Bruschetta et al., 2013). Whether it be through serotonin release from platelets, decreased motivation, or increased survival methods, the mechanisms by which serotonin impacts exercise endurance have yet to be elucidated. 


\section{TRYPTOPHAN SUPPLEMENTATION IN HORSES}

Research in cattle, poultry, swine, dogs, foxes, humans, mice, fish, and monkeys have shown sedative effects, including decreases in aggression, fear, stress, depression, stereotypic behavior, and/or overall activity level, as a result of various doses of tryptophan (as cited in Grimmett and Sillence, 2005). For a review of these studies, see Table 1.1. However, other than slight effects seen in one study, no research has been able to show a behavioral effect in horses. This suggests that there may be a potential difference in tryptophan doses required to achieve specific behavioral effects in horses.

Bagshaw et al. (1994) conducted the first study assessing the behavioral effects of tryptophan supplementation in horses. However, the doses given to horses were less than $1 \%$ of the average amount of tryptophan administered using the commercial supplements available today . These researchers actually found that tryptophan supplementation corresponded with a significant increase in activity both when horses were isolated and when they had visual contact with other horses. However, treatment horses did show some significant decreases in heart rate throughout the behavioral tests and stereotypic behavior in one horse was reduced.

Grimmett and Sillence published a review in 2005 summarizing the current research and evaluating areas of future research in the use of tryptophan as a calmative in horses. Since this time, there has been very little research in supplementing tryptophan to equines. Malmkvist and Christensen (2007) supplemented young horses with a commercial tryptophan product but found no significant differences between treatment and control groups with respect to mean heart rate or behavioral observations taken during a voluntary approach test and handling test. Another study by Noble et al. (2008) looked at the effects of supplementing horses with a commercial dose of Ltryptophan on plasma tryptophan levels and response to approaching a novel object/person. Results 
showed that plasma tryptophan levels increased three-fold as a result of supplementation. The ratio of tryptophan to other large neutral amino acids also increased. However, no significant behavioral effects were noted.

Paradis et al. (1991) evaluated toxicity effects of tryptophan supplementation in horses, either via oral administration or intravenous infusion. They found that plasma tryptophan concentrations peak after dosing and return to pre-dosing levels within 48 hours. These researchers also demonstrated that orally administered tryptophan can be metabolized into 3-methylindole or, more commonly, indole. Both of these compounds may have toxic effects, causing hemolytic anemia and/or respiratory distress. These consequences were seen in one of the four ponies receiving $350 \mathrm{mg}$ tryptophan/ $\mathrm{kg}$ bodyweight and in three of the four ponies receiving $600 \mathrm{mg}$ tryptophan/kg bodyweight.

It is clear from the evidence, or lack thereof, provided by previous research that the safety and efficacy of using tryptophan as a calmative in horses warrants further investigation. However, supplement companies continue to market products containing tryptophan to horse owners, insuring that the ingredient is safe and effective for use in equines. For a review of these products, see Table 1.2. Horse owners find these supplements appealing because they do not require veterinary oversight yet promise a safer, more relaxed, and easy-to-ride horse. 
TABLE 1.1: Review of research supplementing tryptophan to various species

\begin{tabular}{|c|c|c|c|c|}
\hline Author & Species & Treatments & $\begin{array}{l}\text { Supplement } \\
\text { Schedule }\end{array}$ & Results \\
\hline Young et al., 1986 & Human males & $\sim 129 \mathrm{mg} / \mathrm{kg}$ & $1 \mathrm{x}$ & increase self-reported depression symptoms \\
\hline $\begin{array}{l}\text { Liebermann et al., } \\
1986\end{array}$ & Humans & $50 \mathrm{mg} / \mathrm{kg}$ & $1 \mathrm{x}$ & sedative effect \\
\hline $\begin{array}{l}\text { Nakanishi et al., } \\
1997\end{array}$ & Calves & $160 \mathrm{mg} / \mathrm{kg}$ & $7 \mathrm{~d}$ & $\begin{array}{l}\text { calves supplemented with tryptophan showed increased } \\
\text { lying time and decreased exploratory behavior in the } \\
\text { two-weeks post weaning; feed utilization was not } \\
\text { different between treatment and control calves. }\end{array}$ \\
\hline $\begin{array}{l}\text { Laycock \& Ball, } \\
1990\end{array}$ & Chickens & $5000 \mathrm{mg} / \mathrm{kg}$ & $6 \mathrm{~d}$ & $\begin{array}{l}\text { hysteria episodes decreased; feed consumption increased, } \\
\text { egg laying increased; plasma Trp increased }\end{array}$ \\
\hline Shea et al., 1990 & $\begin{array}{l}\text { Male broiler } \\
\text { breeders }\end{array}$ & $\begin{array}{l}\text { control, } 0.38 \% \\
0.75 \%, 1.5 \%\end{array}$ & $\begin{array}{l}4-20 \text { weeks of } \\
\text { age }\end{array}$ & $\begin{array}{l}\text { Birds fed supplemental tryptophan showed less } \\
\text { aggressive pecking; low levels }(0.38 \%) \text { were as effective } \\
\text { as high levels }(1.5 \%)\end{array}$ \\
\hline $\begin{array}{l}\text { DeNapoli et al., } \\
2000\end{array}$ & Dogs & $\begin{array}{l}\text { Low protein, low } \\
\text { Trp; low protein, } \\
\text { high Trp; high } \\
\text { protein, low Trp; } \\
\text { high protein, high } \\
\text { Trp }\end{array}$ & daily, 7d & $\begin{array}{l}\text { No differences in fearfulness between diets; dogs on high } \\
\text { protein diet supplemented with Trp showed less } \\
\text { dominance aggression than dogs feed a high protein diet } \\
\text { without Trp; dogs on low protein diet with supplemented } \\
\text { Trp showed less territorial aggression than dogs fed a } \\
\text { low protein diet without supplemental Trp }\end{array}$ \\
\hline Paradis et al., 1991 & Horses & $350 \mathrm{mg} / \mathrm{kg}$ & $\begin{array}{l}1 \mathrm{x}, \text { feed } \\
\text { withheld for } \\
24 \mathrm{hrs} \text { prior to } \\
\text { admin }\end{array}$ & $\begin{array}{l}\text { Four ponies developed hemoglobinuric nephrosis and } \\
\text { bronchiolar degeneration; one pony also developed } \\
\text { hemolysis, hemoglobinuria, and increased respiratory } \\
\text { rate }\end{array}$ \\
\hline Paradis et al., 1991 & Horses & $100 \mathrm{mg} / \mathrm{kg} \mathrm{IV}$ & $\begin{array}{l}1 \mathrm{x}, \text { feed } \\
\text { withheld for } \\
24 \mathrm{hrs} \text { prior to } \\
\text { admin }\end{array}$ & $\begin{array}{l}\text { Ponies did not show any pathology due to Trp } \\
\text { supplementation }\end{array}$ \\
\hline
\end{tabular}




\begin{tabular}{lll}
\hline Paradis et al., $1991 \quad$ Horses & $600 \mathrm{mg} / \mathrm{kg}$ & $\begin{array}{l}\text { 1x, feed } \\
\text { withheld for } \\
\text { 24hrs prior to } \\
\text { admin }\end{array}$ \\
\hline $\begin{array}{l}\text { Bagshaw et al., } \\
1994\end{array}$ & Horses & $0,0.05, \& 0.1 \mathrm{mg} / \mathrm{kg} \begin{array}{l}1 \mathrm{x}, 2 \mathrm{~h} \text { prior to } \\
\text { testing }\end{array}$
\end{tabular}

Four out of five ponies showed restlessness, increased respiratory rate, hemolysis, and hemoglobinuria

Horses dosed with $0.1 \mathrm{mg} / \mathrm{kg}$ had higher rates of walking/sniffing and heart rate during isolation compared to horses dosed with $0 \mathrm{mg} / \mathrm{kg}$; one horse showed a reduction in sterotypic head twisting after being dosed

\begin{tabular}{|c|c|c|c|c|}
\hline Noble et al., 2008 & Horses & $12.5 \mathrm{mg} / \mathrm{kg}$ & $1 \mathrm{x}$ & $\begin{array}{l}\text { plasma Trp increased, with peak } 1.5-2 \text { hours post-dosing; } \\
\text { supplement made no difference in behavior around novel } \\
\text { object/person }\end{array}$ \\
\hline $\begin{array}{l}\text { Malmkvist \& } \\
\text { Christensen, } 2007\end{array}$ & Horses & $12.9 \mathrm{mg} / \mathrm{kg}$ & $\begin{array}{l}1 \mathrm{x}, 2-3 \mathrm{~h} \text { prior to } \\
\text { test }\end{array}$ & $\begin{array}{l}\text { No difference in heart rate or novel stimulus approach } \\
\text { test }\end{array}$ \\
\hline Farris et al., 1998 & Horses & $\begin{array}{l}100 \mathrm{mg} / \mathrm{kg} \text { IV: } \\
\text { glucose with Trp; no } \\
\text { glucose with Trp; } \\
\text { glucose without Trp; } \\
\text { no glucose, no Trp }\end{array}$ & $1 \mathrm{x}$ & $\begin{array}{l}\text { Horses dosed with tryptophan immediately before } \\
\text { exercise and either glucose or a placebo during exercise } \\
\text { had a lower mean time to exhaustion than horses dosed } \\
\text { with two rounds of placebos; these horses also had } \\
\text { higher plasma prolactin. }\end{array}$ \\
\hline $\begin{array}{l}\text { O'Reilly, } 2006 \\
\text { (Thesis) }\end{array}$ & Horses & $3 \mathrm{~g} 5 \mathrm{x} /$ day & $21 \mathrm{~d}$ & Tryptophan had no effect on cribbing behavior \\
\hline $\begin{array}{l}\text { Adeola \& Ball, } \\
1992\end{array}$ & Pigs & $\begin{array}{l}0,5,10,25 \mathrm{~g} \mathrm{Trp} / \mathrm{kg} \\
\text { diet }\end{array}$ & $5 \mathrm{~d}$ & $\begin{array}{l}\text { doubled plasma Trp; pigs showing higher stress pre- } \\
\text { slaughter had lower hypothalamic serotonin content; ; } \\
\text { reduced PSE score; hypothalamic serotonin } \\
\text { concentration increased with Trp supplementation but } \\
\text { the increase peaks at day } 5 \text { and then decreases: } \\
\text { researchers suspect an adaptive response }\end{array}$ \\
\hline Shen, et al., 2015 & Pigs & $0.0 \% \& 0.8 \%$ & $12 \mathrm{~d}$ & $\begin{array}{l}\text { Piglets fed diets with } 0.8 \% \text { Trp had a higher average } \\
\text { daily gain and feed efficiency and lower salivary cortisol } \\
\text { concentrations after a stressful situation }\end{array}$ \\
\hline
\end{tabular}


$0.0 \%, 0.8 \%, \& 0.7 \%$

$\begin{array}{lll}\text { Shen et al., } 2015 \quad \text { Pigs } & \begin{array}{l}\text { (with the same } \\ \text { amino acid ratios as } 16 \mathrm{~d} \\ 0.8 \%)\end{array}\end{array}$

Koopmans et al., 2005

Pigs

diets with high and normal Trp:LNAA

$7 d$ and $12 d$

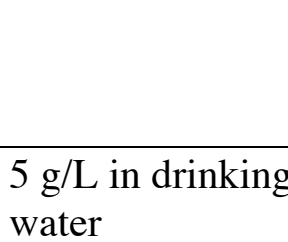
$3 d$

Control, $2 \mathrm{x}$

\begin{tabular}{|c|c|c|c|c|}
\hline Peeters et al., 2004 & Pigs & $\begin{array}{l}5 \mathrm{~g} / \mathrm{L} \text { in drinking } \\
\text { water }\end{array}$ & $3 d$ & $\begin{array}{l}\text { Pigs that received tryptophan spent significantly more } \\
\text { time lying down during simulated transport }\end{array}$ \\
\hline Li et al., 2006 & Pigs & $\begin{array}{l}\text { Control, } 2 \mathrm{x} \\
\text { recommendation, } 4 \mathrm{x} \\
\text { recommendation }\end{array}$ & $7 d, 3 d, 3 d$ & $\begin{array}{l}\text { Trp-supplemented pigs spent more time laying, less time } \\
\text { eating, and less time fighting }\end{array}$ \\
\hline Poletto et al., 2014 & $\begin{array}{l}\text { Pigs (gestating } \\
\text { sows) }\end{array}$ & $\sim 66 \mathrm{mg} / \mathrm{kg}$ & $7 d$ & $\begin{array}{l}\text { Trp-supplemented sows showed less aggression and } \\
\text { increased exploratory behavior when mixed with other } \\
\text { sows }\end{array}$ \\
\hline $\begin{array}{l}\text { Martinez-Trejo } \\
\text { et al., } 2009\end{array}$ & Weaned piglets & $\begin{array}{l}0.23 \%, 0.27 \% \\
0.31 \%, 0.35 \%\end{array}$ & $\begin{array}{l}\text { 3d before and } \\
\text { after weaning } \\
\text { (6d total) }\end{array}$ & $\begin{array}{l}\text { Highest levels of tryptophan supplementation show less } \\
\text { appendage biting and aggression than the two lowest }\end{array}$ \\
\hline $\begin{array}{l}\text { Hilakivi-Clarke et } \\
\text { al., } 1990\end{array}$ & Male mice & $\begin{array}{l}50,75,100,125,200 \\
\mathrm{mg} / \mathrm{kg} \\
\text { intraperitoneal }\end{array}$ & $\begin{array}{l}1 \mathrm{x}, 60 \mathrm{~min} \text { prior } \\
\text { to test }\end{array}$ & $\begin{array}{l}\text { Antidepressant-like effects in Porsolt's Swim Test } \\
\text { At } 125 \text { and } 200 \mathrm{mg} / \mathrm{kg} \text { injection, response was no } \\
\text { different than control }\end{array}$ \\
\hline Janczak et al., 2001 & Mice & $\begin{array}{l}2.08 \mathrm{~g} / \mathrm{L} \text { in drinking } \\
\text { water }\end{array}$ & $\begin{array}{l}\text { dialy for } 2 \\
\text { weeks before } \\
\text { behavior test }\end{array}$ & $\begin{array}{l}\text { Mice treated with tryptophan showed reduced } \\
\text { exploratory behavior in resident-intruder test, number of } \\
\text { fights, and time spent fighting }\end{array}$ \\
\hline
\end{tabular}

recommendation, $4 \mathrm{x} 7 \mathrm{~d}, 3 \mathrm{~d}, 3 \mathrm{~d}$ recommendation

\begin{tabular}{|c|c|c|c|c|}
\hline Peeters et al., 2004 & Pigs & $\begin{array}{l}5 \mathrm{~g} / \mathrm{L} \text { in drinking } \\
\text { water }\end{array}$ & $3 d$ & $\begin{array}{l}\text { Pigs that received tryptophan spent significantly more } \\
\text { time lying down during simulated transport }\end{array}$ \\
\hline Li et al., 2006 & Pigs & $\begin{array}{l}\text { Control, } 2 \mathrm{x} \\
\text { recommendation, } 4 \mathrm{x} \\
\text { recommendation }\end{array}$ & $7 d, 3 d, 3 d$ & $\begin{array}{l}\text { Trp-supplemented pigs spent more time laying, less time } \\
\text { eating, and less time fighting }\end{array}$ \\
\hline Poletto et al., 2014 & $\begin{array}{l}\text { Pigs (gestating } \\
\text { sows) }\end{array}$ & $\sim 66 \mathrm{mg} / \mathrm{kg}$ & $7 d$ & $\begin{array}{l}\text { Trp-supplemented sows showed less aggression and } \\
\text { increased exploratory behavior when mixed with other } \\
\text { sows }\end{array}$ \\
\hline $\begin{array}{l}\text { Martinez-Trejo } \\
\text { et al., } 2009\end{array}$ & Weaned piglets & $\begin{array}{l}0.23 \%, 0.27 \% \\
0.31 \%, 0.35 \%\end{array}$ & $\begin{array}{l}\text { 3d before and } \\
\text { after weaning } \\
\text { (6d total) }\end{array}$ & $\begin{array}{l}\text { Highest levels of tryptophan supplementation show less } \\
\text { appendage biting and aggression than the two lowest }\end{array}$ \\
\hline $\begin{array}{l}\text { Hilakivi-Clarke et } \\
\text { al., } 1990\end{array}$ & Male mice & $\begin{array}{l}50,75,100,125,200 \\
\mathrm{mg} / \mathrm{kg} \\
\text { intraperitoneal }\end{array}$ & $\begin{array}{l}1 \mathrm{x}, 60 \mathrm{~min} \text { prior } \\
\text { to test }\end{array}$ & $\begin{array}{l}\text { Antidepressant-like effects in Porsolt's Swim Test } \\
\text { At } 125 \text { and } 200 \mathrm{mg} / \mathrm{kg} \text { injection, response was no } \\
\text { different than control }\end{array}$ \\
\hline Janczak et al., 2001 & Mice & $\begin{array}{l}2.08 \mathrm{~g} / \mathrm{L} \text { in drinking } \\
\text { water }\end{array}$ & $\begin{array}{l}\text { dialy for } 2 \\
\text { weeks before } \\
\text { behavior test }\end{array}$ & $\begin{array}{l}\text { Mice treated with tryptophan showed reduced } \\
\text { exploratory behavior in resident-intruder test, number of } \\
\text { fights, and time spent fighting }\end{array}$ \\
\hline
\end{tabular}

Pigs fed diets supplemented with Trp had better feed efficiency

Pigs at the higher level of supplementation showed higher plasma Trp, lower basal plasma cortisol, lower basal plasma noradrenaline, but no difference in plasma adrenaline concentrations compared to controls; during social stress, pigs on the higher tryptophan diet showed less avoidance behavior, but similar amounts of physical activity and aggression; post-stress plasma cortisol, noradrenaline, and adrenaline were less in pigs provided supplement.

Pigs that received tryptophan spent significantly more time lying down during simulated transport

Trp-supplemented pigs spent more time laying, less time eating, and less time fighting

Trp-supplemented sows showed less aggression and increased exploratory behavior when mixed with other Highest levels of tryptophan supplementation show less after weaning (6d total) 
Schaechter \&

Wurtman, 1990

Rat

Hypothalamic $2 \mu \mathrm{M}$ Tryptophan

Slices superfused over

$130 \mathrm{~min}$

Superfusing hypothamalic slices with medium containing tryptophan increases total serotonin release by $115.0 \pm$ $5.9 \%$. Superfusing tryptophan in the medium also increases electrically-stimulated total serotonin release by $125.9 \pm 3.9 \%$. In slices with reduced tryptophan levels (due to leucine supplemented medium), total serotonin release decreased by $88.7 \pm 2.0 \%$.

Rats that received tryptophan during the day (8:00), there was an increase in 5-HT and 5-HIAA in the brain; rats

Esteban et al., 2004 Rats $\quad 300 \mathrm{mg}$ 5d that received tryptophan at night (20:00), the 5-HT/5-

HIAA did not change but there was a significant increase in circulating melatonin

\begin{tabular}{|c|c|c|c|}
\hline Basic et al., 2013 & Salmon & $\begin{array}{l}\text { Control diet, } 2 \mathrm{x} \operatorname{Trp}, 7 \mathrm{~d} \\
3 \mathrm{x} \operatorname{Trp}, 4 \mathrm{x} \text { Trp }\end{array}$ & $\begin{array}{l}\text { Trp supplemented fish had lower basal levels of cortisol } \\
1 \text { and } 10 \text { days after supplementation stopped }\end{array}$ \\
\hline $\begin{array}{l}\text { Rouvinen et al., } \\
1999\end{array}$ & Silver Foxes & $1.2 \mathrm{~g} / \mathrm{MJ} \mathrm{ME}$ & $\begin{array}{l}\text { Supplementing Trp increased exploratory behavior in } \\
\text { females; Trp did not have a significant effect on fur } \\
\text { growth, fur quality, or weight gain }\end{array}$ \\
\hline Raleigh, 1987 & $\begin{array}{l}\text { rvet } \\
\text { nkeys }\end{array}$ & $10,20,40 \mathrm{mg} / \mathrm{kg}$ & $\begin{array}{l}\text { Monkeys showed dose-dependent increases in eating, } \\
\text { and decreases in locomotion, vigilance, and aggression }\end{array}$ \\
\hline $\begin{array}{l}\text { Chamberlain et al., } \\
1986\end{array}$ & $\begin{array}{l}\text { Vervet } \\
\text { monkeys }\end{array}$ & $\begin{array}{ll}\text { diets: balanced, Trp- } & \text { 1x; observed } 5 \\
\text { free diet, Trp- } & \text { hours after } \\
\text { supplemented } & \end{array}$ & $\begin{array}{l}\text { Male and females on Trp-supplemented diet showed } \\
\text { reduced competitive aggression; when males were given } \\
\text { a mixture containing no Trp, spontaneous and } \\
\text { competitive aggression increased }\end{array}$ \\
\hline Weld et al., 1998 & $\begin{array}{l}\text { Rhesues } \\
\text { monkeys } \\
\text { (male) }\end{array}$ & $\begin{array}{l}100 \mathrm{mg} / \mathrm{kg} \text {, twice per } 21 \mathrm{~d} \\
\text { day }\end{array}$ & $\begin{array}{l}\text { In monkeys with a history of self-injurious behavior, the } \\
\text { supplement reduced the behavior and increased serotonin } \\
\text { turnover (measured by concentrations of 5-HIAA in } \\
\text { CSF). Serotonin metabolism and behavior were not } \\
\text { affected in monkeys with no history of self-injurious } \\
\text { behavior. }\end{array}$ \\
\hline
\end{tabular}




\section{TABLE 1.2: Summary of paste supplements containing tryptophan available to horse owners in the United States}

\begin{tabular}{|c|c|c|c|}
\hline Product & Dosing Directions & $\begin{array}{l}\text { Dose } \\
(\mathrm{mg} \mathrm{Trp} / \\
\mathrm{kg} \mathrm{BW})^{*}\end{array}$ & Other Ingredients \\
\hline $\begin{array}{l}\text { Divine Equine } \\
\text { (Oralx Corp., Ogden, UT) }\end{array}$ & $\begin{array}{l}\text { administer } 4 \text { hours before } \\
\text { stressful event }\end{array}$ & 3.4 & $\begin{array}{l}\text { valerian root, black cohash, passion flower, ginger root, hops, wood betony, cherry extract for flavoring, benzol alcohol } \\
\qquad .05 \% \text {, sorbic acid as a preservative, xanthan gum }\end{array}$ \\
\hline $\begin{array}{l}\text { SmartCalm Ultra Paste } \\
\text { (SmartPak Equine, } \\
\text { Plymouth, MA) }\end{array}$ & $\begin{array}{l}\text { administer } 2-4 \text { hours before } \\
\text { stressful event }\end{array}$ & 2 & $\begin{array}{l}\text { active: magnesium, taurine, inositol, thiamine, vitamin e } \\
\text { inactive: ascorbyl palmitate, artificial flavor, citric acid, coconut oil, methylparaben, silicon dioxide, soy lecithin, vegetable } \\
\text { oil (cold pressed), vitamin e supplement }\end{array}$ \\
\hline $\begin{array}{l}\text { B-Kalm } \\
\text { (Farnam Companies, Inc., } \\
\text { Phoenix, AZ) }\end{array}$ & $\begin{array}{l}\text { administer } 1.5-2 \text { hours prior } \\
\text { to exercise }\end{array}$ & 20 & $\begin{array}{l}\text { inactive ingredients: dextrose, ethyl alcohol, ground limestone, potassium sorbate, sodium bentonite, sodium benzoate, } \\
\text { sodium saccharin, thiamine hydrochloride with artificial flavor and color, water, and xanthan gum }\end{array}$ \\
\hline $\begin{array}{l}\text { Vision } \\
\text { (VitaFlex Nutrition, Council } \\
\text { Bluffs, IA) }\end{array}$ & $\begin{array}{l}\text { administer } 2 \text { hours before } \\
\text { competition/race/trailering }\end{array}$ & 5 & $\begin{array}{l}\text { active: thiamine, inositol, riboflavin, magnesium, vitamin b6, valerian root extract } \\
\text { inactive: artificial flavoring, corn starch, glucose, glycerin, maltodextrins, methylparaben, propylparaben, silicon dioxide, } \\
\text { sorbic acid, sorbitol, sucrose, water }\end{array}$ \\
\hline $\begin{array}{l}\text { Tryptoplex } \\
\text { (Oralx Corp., Ogden, UT) }\end{array}$ & $\begin{array}{l}\text { administer } 2 \text { hours prior to } \\
\text { event }\end{array}$ & 3.4 & $\begin{array}{l}\text { water, magnesium amino acid hydrochloride, pyridoxine hydrochloride, ginger glycyrrhiza, juniper berries, cherry extract } \\
\text { for flavoring, benzoic acid (a preservative) sorbic acid ( a preservative) xanthan gum }\end{array}$ \\
\hline $\begin{array}{l}\text { Calmex-V } \\
\text { (Med-Vet Pharmaceuticals, } \\
\text { Eden Prarie, MN) }\end{array}$ & $\begin{array}{l}\text { 2-4 hours prior to } \\
\text { trailering/riding when } \\
\text { needed; may be given } 24 \text { and } \\
48 \text { hours prior to } \\
\text { trailering/riding } \\
\end{array}$ & 1.5 & $\begin{array}{l}\text { active: valerian root, thiamine, taurine, inositol } \\
\text { inactive: distilled water, glycerin, sodium propionate, xanthan gum }\end{array}$ \\
\hline $\begin{array}{l}\text { Easy Going } \\
\text { (ProFormula Laboratories, } \\
\text { Inc., Ft. Lauderdale, FL) }\end{array}$ & $\begin{array}{c}\text { administer } 3 \text { hours prior to } \\
\text { event }\end{array}$ & 8.08 & $\begin{array}{c}\text { valerian root, passion flower, kava kava, ginger root, hops, wood betony, ethyl alcohol, aloe vera gel, } .05 \% \text { potassium } \\
\text { sorbate as a persvative, acacia gum }\end{array}$ \\
\hline $\begin{array}{l}\text { Quietex II } \\
\text { (Farnam Companies, Inc., } \\
\text { Phoenix, AZ) }\end{array}$ & $\begin{array}{l}\text { administer } 2 \text { hours before } \\
\text { training or competition }\end{array}$ & 5 & $\begin{array}{l}\text { active: thiamine, inositol, magnesium, vitamin b6, valerian root extract } \\
\text { inactive: corn starch, glucose, glycerin, malt syrup, maltodextrins, methylparaben, propylparaben, silicon dioxide, sorbic } \\
\text { acid, sorbitol, sucrose, water }\end{array}$ \\
\hline $\begin{array}{l}\text { EQUI+Calm } \\
\text { (Equine Healthcare } \\
\text { International, Aberdeen, NC) }\end{array}$ & $\begin{array}{l}\text { administer once the night } \\
\text { before and once the morning } \\
\text { of performance; additional } \\
\text { tubes can be administered at } \\
6-12 \text { hour intervals } \\
\end{array}$ & $\begin{array}{c}\text { not } \\
\text { quantified }\end{array}$ & $\begin{array}{l}\text { active: magnesium, melatonin, arginine, leucine, theanine, thiamine, phenylalanine, bismuth } \\
\text { inactive: maple flavor, praline flavor, deionized water, vitamin c, glycerine, xanthan gum, methyl paraben, propyl paraben, } \\
\text { acesulfame } \mathrm{k} \text { sweetner }\end{array}$ \\
\hline $\begin{array}{l}\text { Perfect Prep EQ Extreme } \\
\text { Paste } \\
\text { (Perfect Products, LLC, } \\
\text { Morrow, OH) }\end{array}$ & $\begin{array}{l}\text { feed } 90 \text { minutes before } \\
\text { increased stress; effects } \\
\text { begin within } 1 \text { hour and last } \\
6-8 \text { hours }\end{array}$ & $\begin{array}{c}\text { not } \\
\text { quantified }\end{array}$ & $\begin{array}{c}\text { active: magnesium, inositol, thiamine } \\
\text { proprietary blend: soybean oil, magnesium amino acid chelate, thiamine mononitrate, soy lecithin, inositol, silica gel, } \\
\text { vitamin e supplment, citric acid, natural and artificial flavors, polysorbate } 80 \text {, ascorbyl palmitate, methylparaben, coconut } \\
\text { oil }\end{array}$ \\
\hline
\end{tabular}




\begin{tabular}{|c|c|c|c|}
\hline $\begin{array}{l}\text { Perfect Prep EQ Supreme } \\
\text { Paste } \\
\text { (Perfect Products, LLC, } \\
\text { Morrow, OH) }\end{array}$ & $\begin{array}{l}\text { administer } 90 \text { minutes prior } \\
\text { to activity; adjust for desired } \\
\text { results; effects begin } 1 \text { hour } \\
\text { after administration and can } \\
\text { last up to } 6 \text { hours }\end{array}$ & $\begin{array}{c}\text { not } \\
\text { quantified }\end{array}$ & $\begin{array}{c}\text { active: magnesium, inositol, thiamine, } 1 \text { tryptophan, vitamin "behave" (a proprietary blend of b vitamins) } \\
\text { proprietary blend: soybean oil, magnesium amino acid chelate, thiamine mononitrate, soy lecithin, inositol, silica gel, } \\
\text { vitamin e supplement, citric acid, natural \& artificial flavors, pyrodoxine hcl, riboflavin, ascorbyl palmitate, methylparaben } \\
\text { coconut oil }\end{array}$ \\
\hline $\begin{array}{l}\text { Oxy-Calm } \\
\text { (Meal and More, Inc., } \\
\text { Morrice, MI) }\end{array}$ & $\begin{array}{l}\text { feed } 2 \text { hours before event; } \\
\text { can be given again } 4 \text { hours } \\
\text { later }\end{array}$ & $\begin{array}{c}\text { not } \\
\text { quantified }\end{array}$ & $\begin{array}{c}\text { vitamin e supplement, sugars, salt, flavorings, vegetable oil, tryptophan, thiamine mononitrate, guar gum, xanthan gum, } \\
\text { wheat germ oil, dried active yeast, lactobacillus acidophilus fermentation product }\end{array}$ \\
\hline $\begin{array}{l}\text { At-Ease Megadose } \\
\text { (Richdel, Inc., Carson City, } \\
\text { NV) }\end{array}$ & $\begin{array}{c}\text { administer one dose } 1-3 \\
\text { hours before and one dose } \\
\text { immediately before desired } \\
\text { event }\end{array}$ & 2 & $\begin{array}{l}\text { soy oil, magnesium oxide, corn starch, thiamine mononitrate, salt, dextrose, pyridoxine hydrochloride, methyl \& propyl } \\
\text { paraben (a preservative), silicon dioxide, artificial apple flavoring }\end{array}$ \\
\hline $\begin{array}{l}\text { Calming Oral Gel } \\
\text { (Kaeco Group Inc., } \\
\text { Savannah, MO) }\end{array}$ & $\begin{array}{l}\text { administer } 2-4 \text { hours prior to } \\
\text { competition, event, } \\
\text { transporting, etc. }\end{array}$ & 3.4 & $\begin{array}{c}\text { valerian root, black cohosh, passion flower, ginger root, hops, wood betony, apple flavor, benzyl alcohol } .05 \% \text {, sorbic acid } \\
\text { as a preservative, xanthan gum }\end{array}$ \\
\hline $\begin{array}{l}\text { Formula Calm B } \\
\text { (dac, Dover, Ohio) }\end{array}$ & $\begin{array}{l}\text { give one to three times daily } \\
\text { before and during events }\end{array}$ & 0.5 & $\begin{array}{l}\text { magnesium sulfate, taurine, thiamine mononitrate, inositol, gylcerin, silica gel, soybean oil, coconut oil, natural \& artificial } \\
\text { flavors }\end{array}$ \\
\hline $\begin{array}{l}\text { Tryptophan Plus Gel } \\
\text { (Horses Prefer, Menomonie, } \\
\text { WI) }\end{array}$ & $\begin{array}{l}\text { feed } 1.5 \text { to } 2 \text { hours prior to } \\
\text { competition, strenuous } \\
\text { exercise, racing, or shipping }\end{array}$ & $\begin{array}{c}\text { not } \\
\text { quantified }\end{array}$ & $\begin{array}{l}\text { dextrose, cane molasses, propylene glycol, silicon dioxide, polysorbate } 80 \text {, niacinamide, pork peptone, calcium chloride, } \\
\text { magnesium oxide, potassium chloride, thiamine hydrochloride, pyridoxine hydrochloride, riboflavin, lactic acid, apple } \\
\text { flavor, methylparaben, propylparaben, and ethoxyquin }\end{array}$ \\
\hline
\end{tabular}

*calculations based on $500 \mathrm{~kg}$ horse 


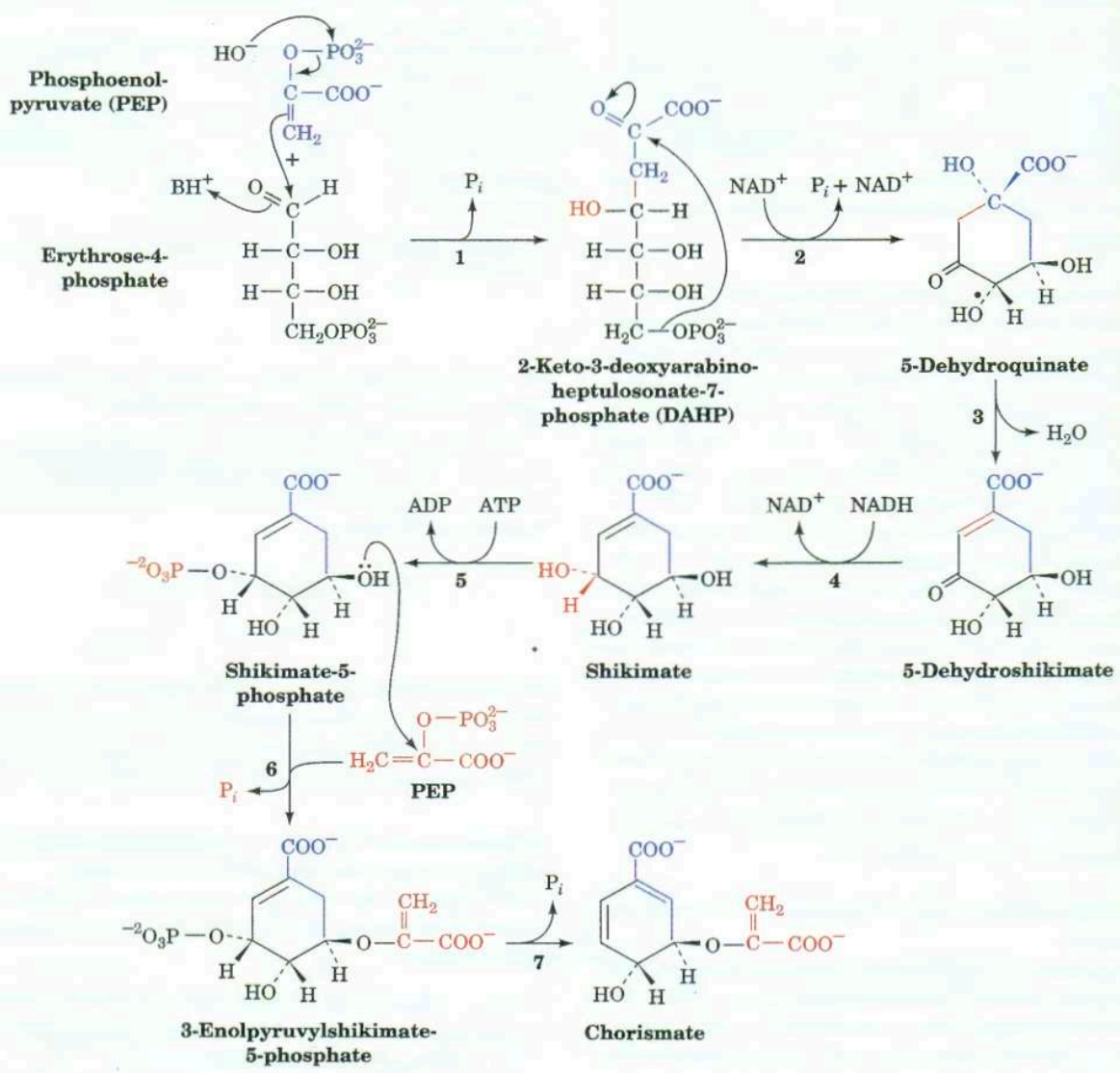

Fig. 1.1: The biosynthesis of chorismate. Pathway enzymes: (1) 2-keto-3-deoxy-Darabinoheptulosonate-7phosphate synthase, (2) dehydroquinate synthase, (3) 5dehydroquinate dehydratase, (4) shikimate dehydrogenase, (5) shikimate kinase, (6) 3-enoylpyruvylshikimate-5-phosphate synthase, (7) chorismate synthase. Reprinted from "Biochemistry" (p. 774), by D. Voet and J.G. Voet, 1995, New York: John Wiley \& Sons, Inc. Copyright 1995 by John Wiley \& Sons, Inc.. Reproduced with permission. 


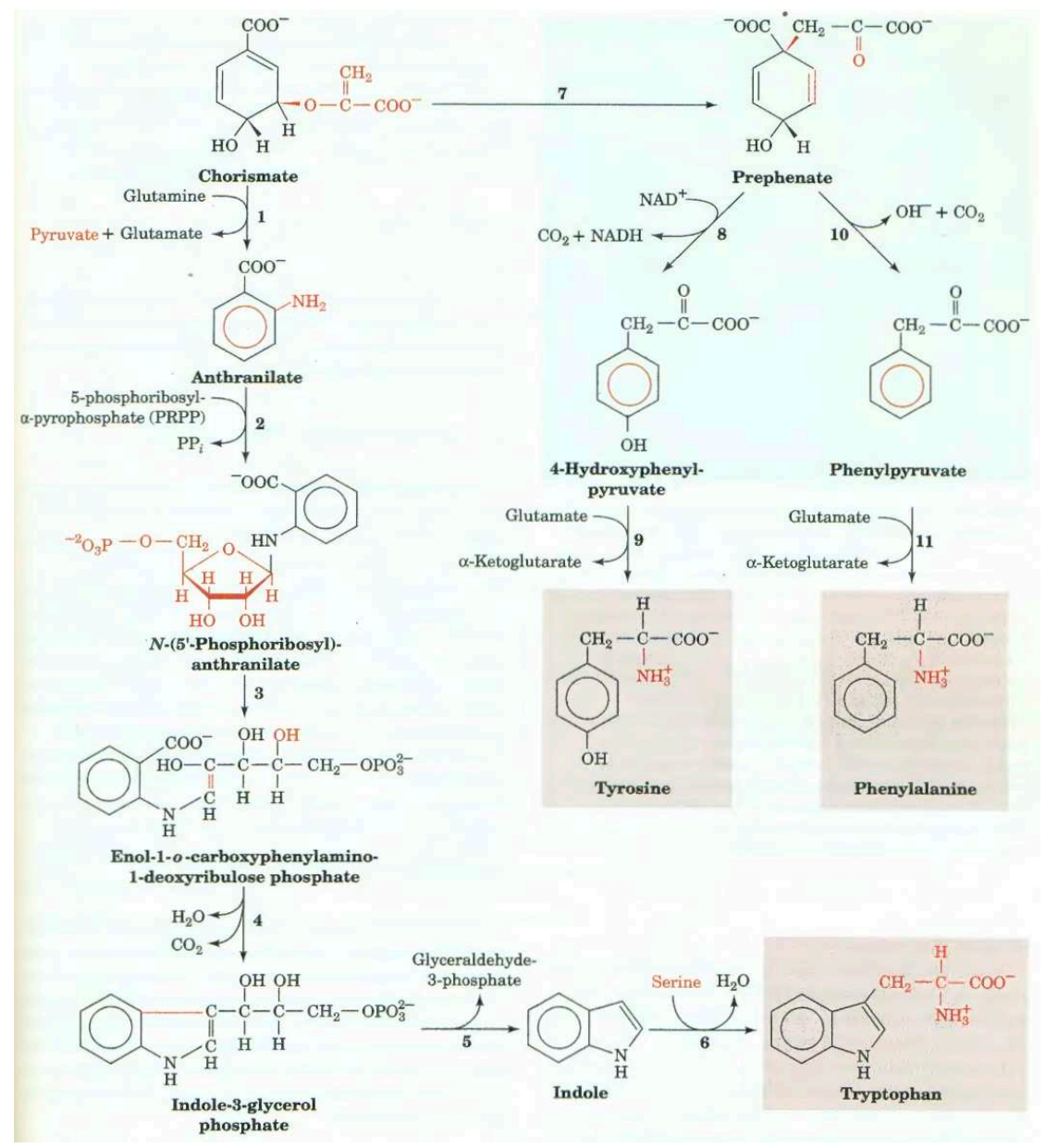

Fig. 1.2: Pathways for the synthesis of tryptophan. Pathway enzymes: (1) anthranilate synthase, (2) anthranilate-phosphoribosyl transferase, (3) $\mathrm{N}-\left(5^{\prime}\right.$ phosphoribosyl)-antranilate isomerase, (4) indole-3-glycerol phosphate synthase, (5) tryptophan synthase, $\alpha$ subunit, (6) tryptophan synthase, $\beta$ subunit. Reprinted from "Biochemistry" ( $p .775)$, by D. Voet and J.G. Voet, 1995, New York: John Wiley \& Sons, Inc. Copyright 1995 by John Wiley \& Sons, Inc.. Reproduced with permission. 

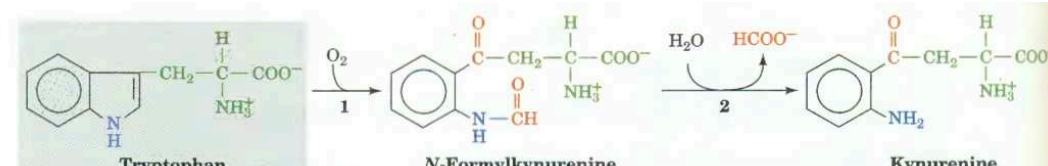

$N$-Formylkynurenine
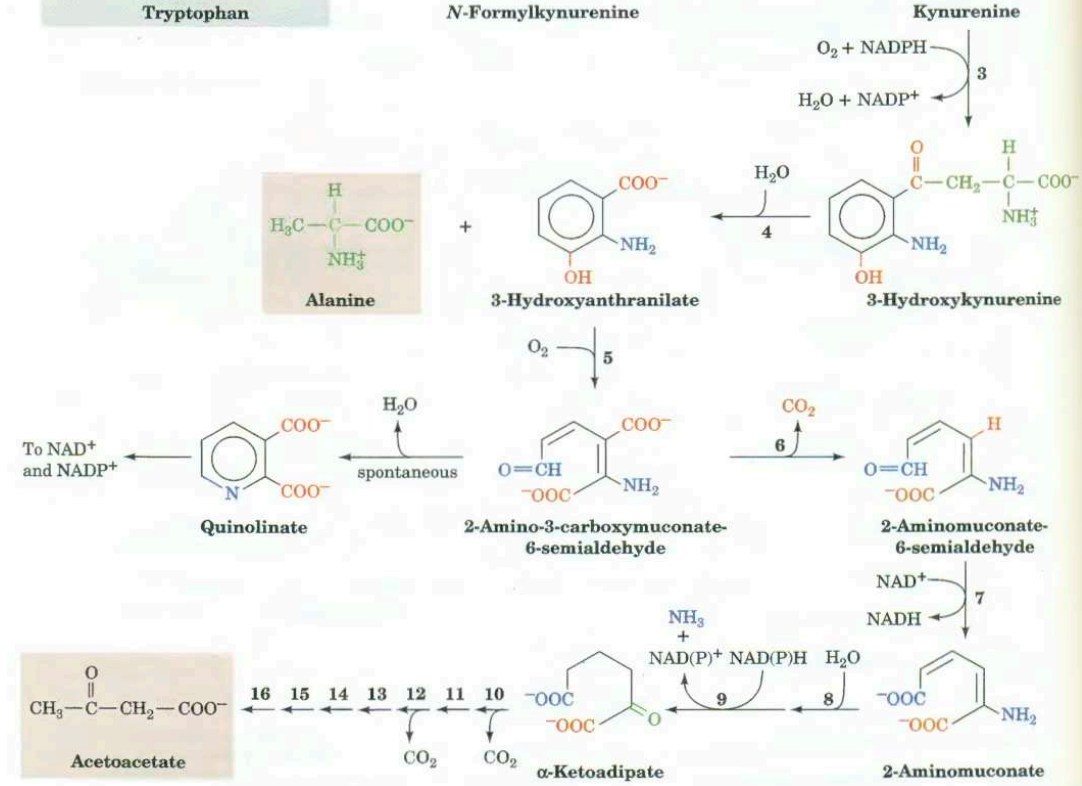

Fig. 1.3: Pathways of tryptophan degradation. Enzymes: (1) tryptophan2,3-dioxygenase, (2) formamidase, (3) kynurenine-3-monooxygenase, (4) kynureninase, PLP dependent, (5) 3-hydroxyanthranilate-3,4,-dioxygenase, (6) amino caroxymuconate semialdehyde decarboxylase, (7) aminomuconate semialdehyde dehydrogenase, (8) hydratase, (9) dehydrogenase, (10) $\alpha$-keto acid dehydrogenase, (11) glutaryl-CoA dehydrogenase, (12) decarboxylase, (13) enoyl-CoA hydratase, (14) $\beta$ hydroxyacyl-CoA dehydrogenase, (15) HMG-CoA synthase, (16) HMGCoA lyase. Reprinted from "Biochemistry" (p. 744), by D. Voet and J.G. Voet, 1995, New York: John Wiley \& Sons, Inc. Copyright 1995 by John Wiley \& Sons, Inc.. Reproduced with permission. 


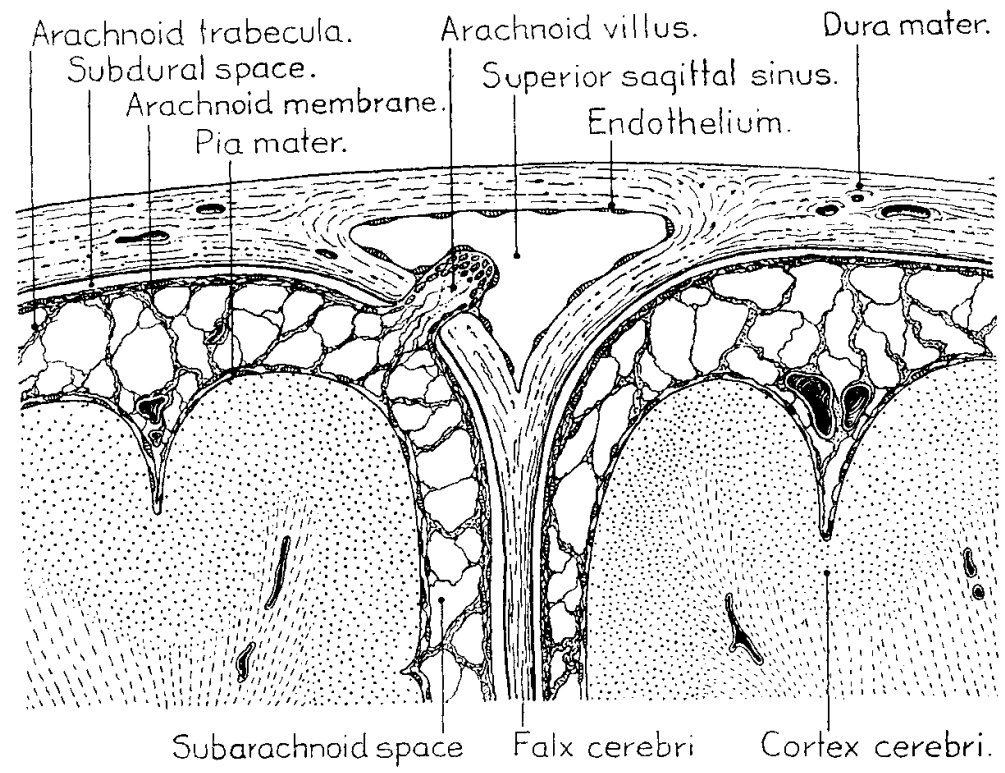

Fig. 1.4: The subarachnoid space. When there is no difference in pressure between the subarachnoid space and the venous blood, there are numerous microvilli made up of layers of overlapping endothelial cells on the arachnoid membrane. Reprinted from "The absorption of cerebrospinal fluid into the venous system," by L.W. Weed, 1923, American Journal of Anatomy, 31(3), 202. Copyright 1923 by Wiley-Liss. Reproduced with permission.

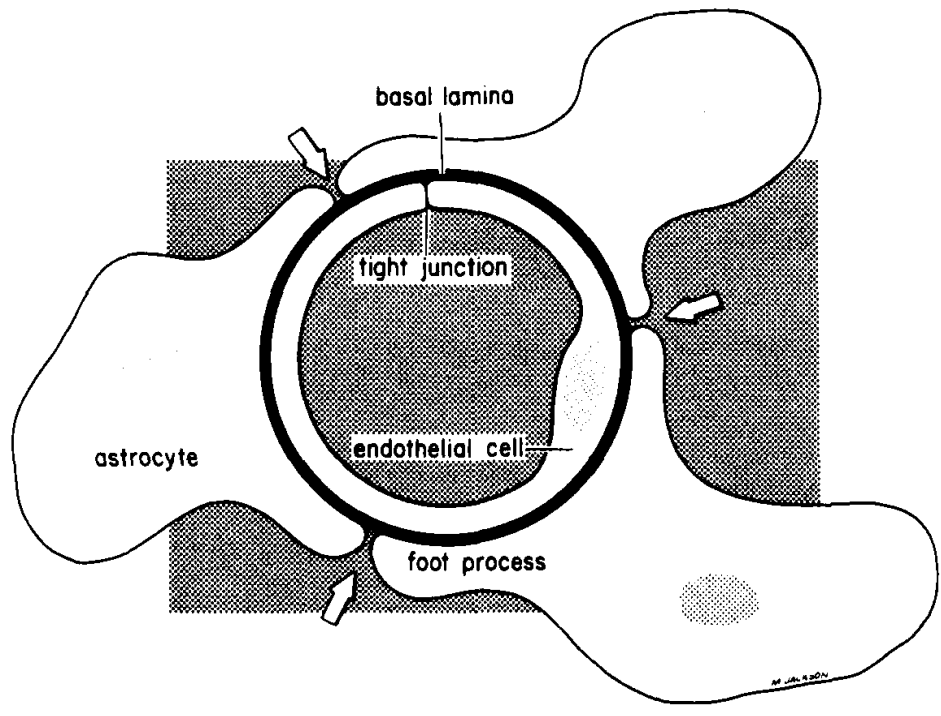

Fig. 1.5: Brain capillary-glial cell junction. The endothelial cells in the brain capillaries are closely associated with astrocytes. Interstitial fluid has access at the locations indicated by the arrows. Reprinted from "Endothelial cell-astrocyte interactions: a cellular model of the blood-brain barrier," by G.W. Goldstein, 1988, Annals of the New York Academy of Sciences, 529(1), 32. Copyright 2006 by John Wiley and Sons. Reproduced with permission. 


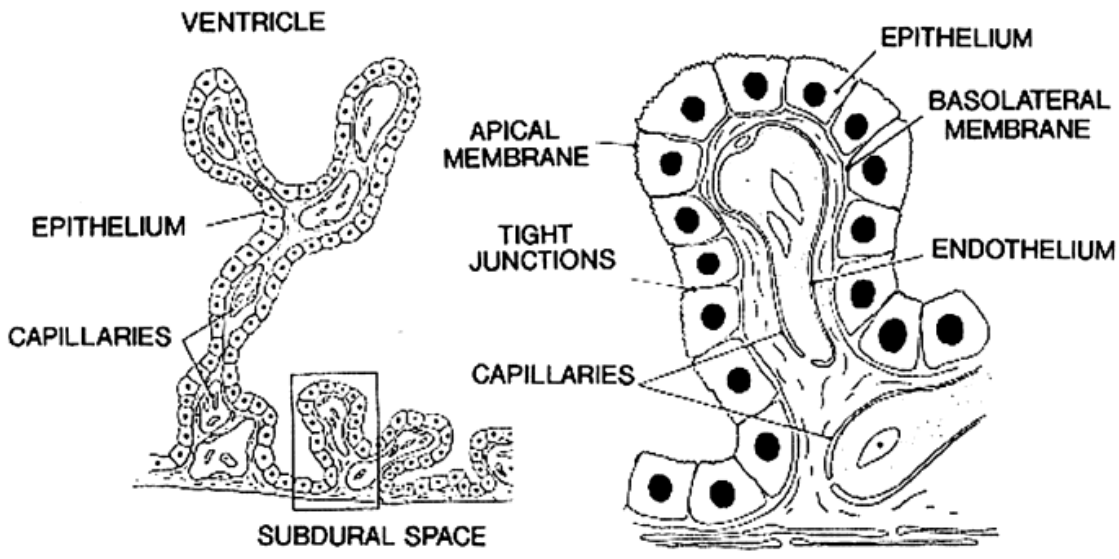

Fig. 1.6: The choroid plexus. The basal lamina of the choroid plexus faces highly vascularized connective tissue. There are microvilli on the apical surface, which face into the cerebrospinal fluid; tight junctions regulate what compounds diffuse from the capillaries into the cerebrospinal fluid. Reprinted from "Mechanisms of CSF secretion by the choroid plexus" by T. Speake, C. Whitwell, H. Kajita, A. Majid, and P.D. Brown, 2001, Microscopy Research and Technique, 52(1), 50. Copyright 2001 by Wiley-Liss. Reproduced with permission.

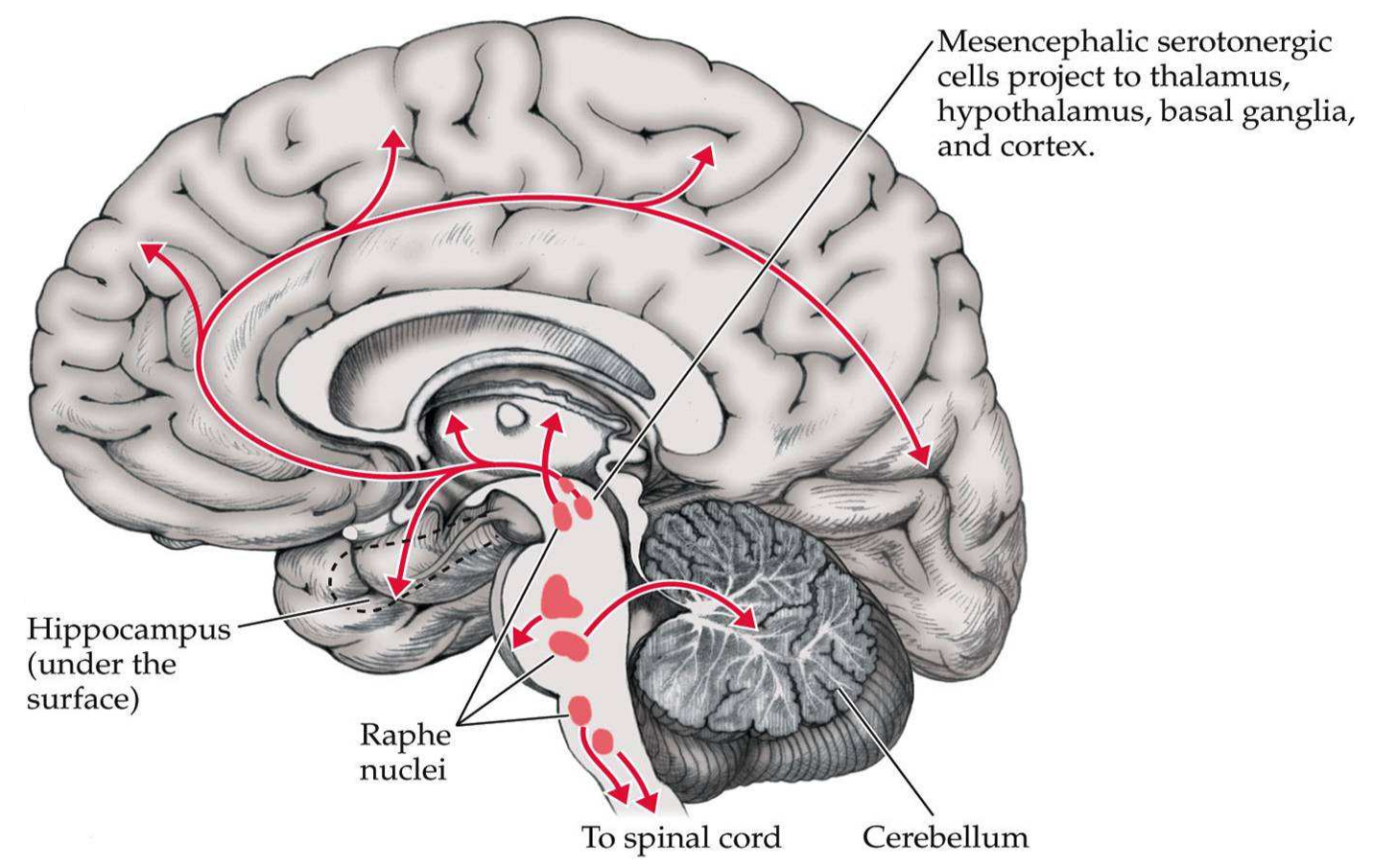

Fig. 1.7: Projections of serotonergic neurons. These neural pathways are involved in various aspects of cognition and behavior: prefrontal cortex (mood), basal ganglia (movement, potentially obsessions and compulsions), limbic area (anxiety and panic), and hypothalamus (appetite and eating behavior) (Corr, 2006). Reprinted from "Biological Psychology: An Introduction to Behavioral, Cognitive, and Clinical Neuroscience” (p.93), by S.M. Breedlove, N.V. Watson, and M.R. Rosenzweig, 2010, Sunderland, MA: Sinauer Associates. Copyright 2010 by Sinauer Associates, Inc.. Reproduced with permission. 
<smiles>[NH3+]C(Cc1c[nH]c2ccccc12)C(=O)[O-]</smiles>

Tryptophan

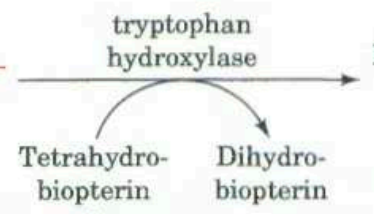

$+$

$\mathrm{O}_{2}$<smiles>[NH3+]C(Cc1c[nH]c2ccc(O)cc12)C(=O)[O-]</smiles>

5-Hydroxytryptophan
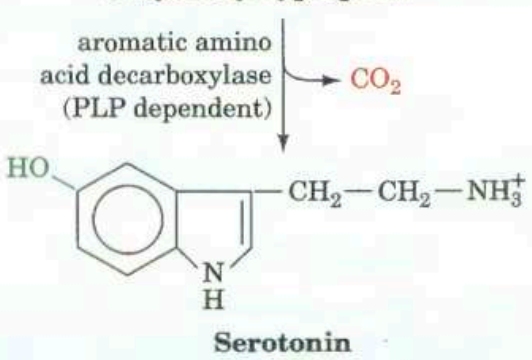

Fig. 1.8: Steps in serotonin biosynthesis. Reprinted from "Biochemistry" (p. 759), by D. Voet and J.G. Voet, 1995, New York: John Wiley \& Sons, Inc. Copyright 1995 by John Wiley \& Sons, Inc.. Reproduced with permission.

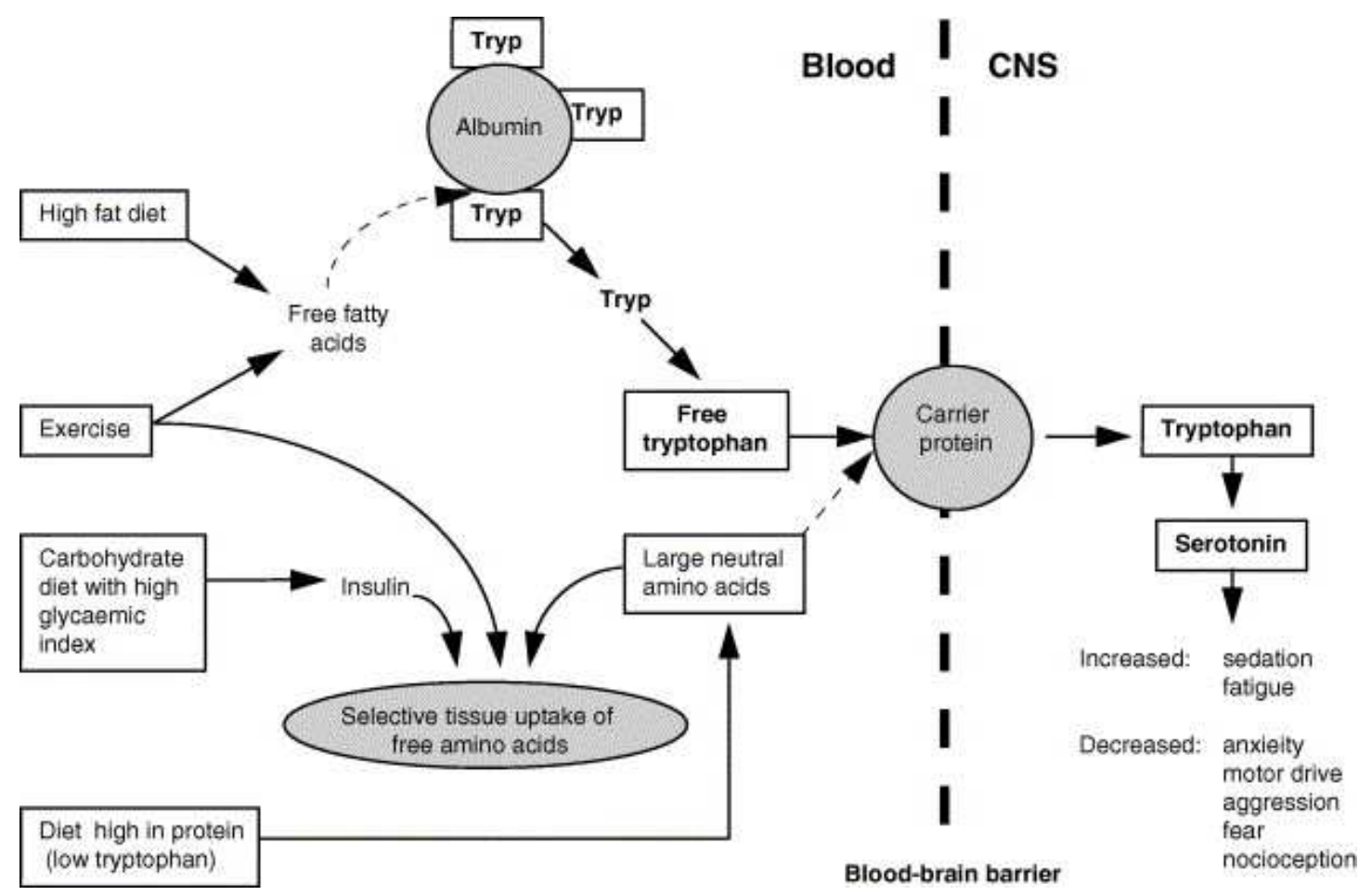

Fig. 1.9: Exercise and diet composition impact the rate of serotonin biosynthesis. Reprinted from "Calmatives for the excitable horse: A review of L-tryptophan," by A. Grimmett and M.N. Sillence, 2005, The Veterinary Journal, 170(1), 26. Copyright 2004 Elsevier Ltd.. Reproduced with permission. 


\section{CHAPTER II: EVALUATING THE EFFECTIVENESS OF VARYING DOSES OF SUPPLEMENTAL TRYPTOPHAN AS A CALMATIVE IN HORSES}

\section{INTRODUCTION}

Tryptophan is often marketed in the horse industry as a calmative supplement, claiming to encourage focus and relaxation while reducing tense, nervous, and spooky behavior. Horse owners frequently utilize calming products in situations including transportation, competition, and other novel training events. Tryptophan is the amino acid precursor to serotonin, a neurotransmitter involved in mood, appetite, sleep, memory, and learning in many species, including humans. Psychiatrists utilize serotonin-enhancing drugs in people to treat conditions such as depression, anxiety, and obsessive-compulsive disorder. Tryptophan supplementation has been shown to increase serotonin production (Fernstrom, 2013). However, while supplementation has been linked to reduced aggression and fearfulness in species other than the horse, there is no current research within the scientific community to support any behavioral effects of tryptophan supplementation in horses (Grimmett and Sillence, 2005). The use of this supplement for calming purposes in horses remains unproven and somewhat controversial among horse owners and professionals. The objective of the following study is to evaluate behavioral and physiological responses resulting from differing dosages of L-tryptophan supplementation in the horse. Identification of a safe and effective dose of tryptophan in horses could give owners a non-prescriptive, non-invasive tool to manage potentially dangerous situations. In addition, feed and supplement companies may benefit from the results of this study, recognizing new dosages or potential additives in products marketed to horse owners.

Because tryptophan competes with other amino acids to bind to transport proteins and cross the blood-brain barrier, scientists are now using the ratio of tryptophan to other large neutral amino 
acids (LNAA) to estimate serotonin production in the central nervous system (Wilson et al., 2007). Research has shown that serum tryptophan levels increase when horses are supplemented at a dose comparable to those used in commercial supplements but that there are no significant behavioral effects (Malmkvist and Christensen, 2007; Noble et al., 2008). Other studies have noticed a potential effect of diet and time of sampling on serum tryptophan levels in horses (Alberghina et al., 2010b; Wilson et al., 2007). While it is possible that these studies may not have seen behavioral effects when supplementing tryptophan because researchers were administering doses too low, there are also health concerns when supplementing tryptophan at doses too high. High doses of tryptophan may cause hemolytic anemia and symptoms of respiratory distress in horses. Researchers predict that these symptoms are the effect of the toxic indole metabolite produced in the hindgut of horses when large doses of tryptophan are administered orally (Paradis et al., 1991). There is a large range, from approximately $12.9 \mathrm{mg} / \mathrm{kg}$ bodyweight (Malmkvist and Christensen, 2007) to $350 \mathrm{mg} / \mathrm{kg}$ bodyweight (Paradis et al., 1991), of oral tryptophan supplementation that has not been scientifically tested for behavioral or physiological effects in horses. Nevertheless, horse owners and trainers frequently utilize supplements containing tryptophan and/or ingredients like magnesium, vitamin $\mathrm{B}_{6}$, and a variety of herbs to promote a calming effect. One benefit to supplementing natural ingredients versus using prescription drugs is that many common dietary components are legal to use in show circuits whereas sedatives are banned substances (USEF, 2015). We hypothesized that, within the untested range of tryptophan supplemented to horses, there is a safe and behaviorally effective dose. 


\section{MATERIALS AND METHODS}

\section{Horses and Care}

The study took place December 2014-January 2015 at Colorado State University's Equine Teaching and Research Center. Ten geldings and two mares were included in the experiment. Three of the horses were Quarter Horse type and the other nine were draft horse crosses. Horses ranged in age from 2.5-16 years (average 9.1 years), height from 150-169 cm (average $158.7 \mathrm{~cm}$ ), weight from 462-626 kg (average $541.4 \mathrm{~kg}$ ), and body condition score from 3-8 (average 5.2) (Henneke et al., 1983). Horses were divided into three groups based on bodyweight. One horse from Group 2 was removed from the study during the acclimation period due to handling difficulties. A description of the remaining horses is provided in Table 2.1. Variance between subjects is displayed in Table 2.2. All horses were used for trail riding and leased from a private owner. Informed client consent was obtained prior to the beginning of the experiment. In addition, all animal care and experimental procedures were approved by the Colorado State University Animal Care and Use Committee under protocol \#14-5191A.

All horses were dewormed with $1.87 \%$ ivermectin (MWI, Boise, ID) and evaluated by a veterinarian prior to being accepted into the study. Throughout the study, horses were allowed ad libitum access to water and salt. Expected body weight at a body condition score of 5 was calculated for each horse (Henneke et al., 1983). NRC (2007) requirements were calculated based on expected body weight. Horses were fed $0.5 \%$ of their expected body weight in concentrate, on a dry matter basis, per day. The concentrate provided was a commercially-available senior feed (Purina Animal Nutrition LLC, Shoreview, MN). Each horse's remaining energy requirements were met with grass hay. Horses were housed in stalls and their daily ration divided into two 
feedings at approximately 07:00 and 19:00 hours. Grain and hay nutrient profiles are reported in Table 2.2.

In order to monitor horse health status, resting respiratory rates were monitored at feeding and blood collection times (three times daily) to safeguard against any signs of respiratory distress going unnoticed. Resting heart rate and rectal temperature were recorded during the effective dose time for horses receiving treatment.

Study Design

Horses were given a 7-day acclimation period before treatment began. There were four treatment levels: a negative control $(\mathrm{CON})$, in which horses received no supplemental tryptophan; LOW, in which horses were given $20 \mathrm{mg}$ Trp/kg BW; MED, in which horses were given $40 \mathrm{mg}$ Trp/kg BW; and HIGH, in which horses were given 60 mg Trp/kg BW. Pharmaceutical grade Ltryptophan (Ajinomoto North America, Raleigh, NC) was mixed with approximately $50 \mathrm{cc}$ applesauce and water to create a paste-like consistency and administered orally before morning feeding. CON horses received an oral dose of only applesauce and water. Each treatment period lasted three days and there was a four-day washout period between treatments. Each horse received all treatments. Horses were assigned treatments in a random order so that all horses in one group were receiving different treatments. To ensure accurate dosing, each horse was weighed on a scale the morning prior to beginning a new treatment. In order to maintain consistent dose, sample collection, and behavior test timing, groups began the experiment on staggered start dates, spaced one to three days apart. Daily procedures, broken down by group, are described in Table 2.3.

\section{Behavior Testing}

Horses underwent a reactivity test similar to one described by Noble et al. (2013). The setup for the behavior test is shown in Figure 2.1. Footing within the chute was raked between 
startle tests in order to try to maintain consistency despite weather conditions. Temperature, wind speed, and precipitation were recorded at the time of each test so that the effect of environmental conditions could be quantified. Each horse was individually led into the chute and held at a standstill for 10 seconds at a location 1 meter into the chute. Upon release, a startling sound and movement were made from a constant location behind the horse. A blind was set up to ensure that horses did not see the startling visual cue prior to stimulus presentation. The rate $(\mathrm{m} / \mathrm{s})$ at which the horse exited the chute (ExitSpeed) was measured using electronic timers (FarmTek, Wylie, TX) placed 6 meters apart on the sides of the chute. Sensors were placed 1 meter off the ground so that the horses' legs or chest may break the light beam. In order to reduce habituation, the startling stimuli rotated between a whoosh paired with a waving flag, an alarm sound paired with an opening umbrella, and machine gun fire paired with a flapping plastic bag. The auditory stimuli were pre-recorded and consistent in volume and intensity. To minimize habituation to the startle test, null tests, in which there are no startling stimuli, were performed during the acclimation period, on day two of each treatment, and on washout days. Startle tests performed 2-3 hours after morning administration of tryptophan supplement on days one and three of each treatment. Each horse experienced either a startle or null test each day. All tests on treatment days were video recorded.

Heart rate data was collected during the behavior tests using a Polar RS800CX training computer, which received heart rate data from a Polar WearLink W.I.N.D. transmitter attached to a chest strap that fitted around the horses' heart girth (Polar Electro OY, Kempele, Finland). Hair around the location of the electrodes was shaved and water and/or electrode gel were used to improve contact with the horses' skin. Horses remained in the stall until a consistent baseline heart rate was found by the heart rate monitor. After the behavior test, each horse was brought back to 
their stall. Handlers waited for heart rate to return to baseline before removing the heart rate monitor.

Heart rate data was uploaded daily via infrared communication to Polar WebLink and transferred to polarpersonaltrainer.com for storage. In addition to the baseline heart rate information, the following measurements were taken: (1) HR30: heart rate (bpm) 30 seconds poststartle stimuli, (3) HR_Diff: HR30 minus baseline heart rate, and (4) TimetoBL: time (sec) from presentation of startle stimuli until baseline heart rate resumed.

\section{Blood Sample Collection and Analysis}

Blood samples were collected into evacuated blood collection tubes containing either a clot activator or sodium fluoride (BD Vacutainer evacuated blood collection tube, Fisher Health Care, Chicago, IL). Blood samples were taken before morning feeding or supplementation (BC1), at approximately 0600, and again before horses preformed the behavior test (BC2). This was done on the last day of the acclimation period as well as throughout the treatment periods. Blood obtained from $\mathrm{BC} 1$ was analyzed for packed cell volume in order to monitor the health of each individual horse, ensuring that no horses became anemic during the study. On days one and three of each treatment, additional blood samples were taken both before the startle test was performed (BC2) and again approximately 15 minutes after the test (BC3). These samples were analyzed to compare glucose, lactate, and cortisol levels before and after the startle test. Blood samples were allowed to clot at room temperature for 45-60 minutes before being centrifuged at 2,500 rpm for 15 minutes. Serum was then removed and stored in $0.5 \mathrm{~mL}$ aliquots in microcentrifuge tubes at $20^{\circ} \mathrm{C}$ until further analysis.

BC2 and BC3 samples were analyzed for serum glucose and lactate using a YSI Model 2700 SELECT Biochemistry Analyzer (YSI Life Sciences, Yellow Springs, Ohio). Serum glucose 
(mg/dL) was analyzed from sodium fluoride vacutainers. Each sample was run 2-4 times, until glucose concentrations read within $1 \%$ of each other. The closest two readings were then averaged together to obtain pre- and post-startle test serum glucose values (from BC2 and BC3, respectively). Post-startle serum glucose (GluPostAvg) was considered as a response variable. Additionally, the difference in glucose values (GluDiff) was calculated by subtracting pre-startle (BC2) values from post-startle (BC3) values. Serum lactate (mmol/L) was analyzed from vacutainers containing only a clot activator. Samples were run 2-4 times, until values within 2\% of each other were obtained. In the same way glucose response variables were obtained, poststartle serum lactate (LactPostAvg) and the difference between lactate pre- and post-startle values (LactDiff) were calculated response variables. The YSI auto-calibrated every five samples. Standards were analyzed before any samples, after every fifty samples, and once all samples had been analyzed. The date each sample was analyzed was recorded and considered as a covariate in the appropriate model (i.e. glucose sample run date (GluRunDate) was considered as a covariate for GluPostAvg and GluDiff models while lactate sample run date (LactRunDate) was considered as a covariate for LactPostAvg and LactDiff models).

Serum cortisol (mg/dL) was analyzed from BC2 and BC3 red top vacutainers using ELISA kits (Rocky Mountain Diagnostics, Colorado Springs, CO). Samples were analyzed in duplicates. Average post-startle serum cortisol values were calculated from BC3 samples (CortPostAvg). In addition, differences in serum cortisol (CortDiff) were calculated by subtracting BC2 values from those found in $\mathrm{BC} 3$ on the startle test days of each treatment. Intra-assay precision averaged an $11.36 \%$ coefficient of variation. The ELISA plate that each sample was run on (CortPretestPlate or CortPosttestPlate) were considered as potential significant predictor variables for CortPostAvg and CortDiff models. 
A subset of samples (from horses in Group 1) were analyzed for serum amino acid content via gas chromatography using the procedures described by Zhang et al. (2005). This was done in order to verify treatment effect and to compare values with those found in previous studies. Concentrations $(\mathrm{mmol} / \mathrm{L})$ of serum free tryptophan (Trp), isoleucine, leucine, tyrosine, phenylalanine, and valine were obtained from pre- and post-supplementation blood draws (BC1 and $\mathrm{BC} 2$, respectively) on Day 1 and Day 3 of treatment. Difference in serum free tryptophan (TrpDiff) was calculated by subtracting values in $\mathrm{BC} 1$ from those found in $\mathrm{BC} 2$. Additionally, the ratio of tryptophan to other large neutral amino acids (Trp:LNAA) was calculated for BC1 and $\mathrm{BC} 2$ samples. The difference between the ratio seen in $\mathrm{BC} 1$ and $\mathrm{BC} 2$ (TrpDiff) was considered as another response variable.

Statistical Analysis

All analyses were carried out using SAS (version 9.4, SAS Inst. Inc., Cary, NC). Significance was set at $p \leq 0.05$ while trends were identified at $p \leq 0.10$.

Day 1 and Day 3 data were analyzed separately. Correlations were run to identify potential covariates for each response variable (ExitSpeed, HR30, HR_Diff, TimetoBL, GluDiff, GluPostAvg, LactDiff, LactPostAvg, CortDiff, and CortPostAvg) using PROC CORR. Potential covariates included the following: Group (1-3); TimeLag, which was the time (in minutes) from tryptophan supplementation until the behavior test was performed; TrtNum, which was the sequence (1-4) of treatments; as well as Temperature $\left({ }^{\circ} \mathrm{C}\right)$ and WindSpeed $(\mathrm{m} / \mathrm{s})$ outside when each behavior test was performed. Correlations between these variables and the response variables were examined; a covariate was included in the models for both Day 1 and Day 3 if a significant correlation to the response variable was found on either day, as long as significance was maintained once added to the model. Additional covariates (GluRunDate, LactRunDate, CortPretestPlate, and 
CortPosttestPlate) were considered for response variables requiring lab analysis. ANOVA and ANCOVA models were constructed for each response variable using PROC MIXED. In all models, Horse and Treatment were identified as class variables. If Group, TrtNum, GluRunDate, LactRunDate, CortPretestPlate, or CortPosttestPlate were included in any model, they were also categorized as class variables. Additionally, Horse was treated as random effect. Treatment means, standard errors, and differences between treatments were determined using the PDIFF option in

\section{LSMEANS.}

The following models were used to evaluate treatment effect on Day 1 and Day 3:

$$
\begin{aligned}
& \text { ExitSpeed = Group }+ \text { Temperature }+ \text { Treatment } \\
& \text { HR30 = TrtNum }+ \text { Treatment } \\
& \text { HR_Diff = Group }+ \text { TrtNum }+ \text { Treatment } \\
& \text { TimetoBL = Temperature }+ \text { Treatment } \\
& \text { GluDiff = GluRunDate }+ \text { TimeLag + Treatment } \\
& \text { GluPostAvg = TimeLag + Treatment } \\
& \text { LactDiff = Treatment } \\
& \text { LactPostAvg = Treatment } \\
& \text { CortDiff = Treatment } \\
& \text { CortPostAvg = CortPosttestPlate + Treatment }
\end{aligned}
$$

The subset of the blood samples ( $n \leq 4$ samples per treatment, day, and blood collection) analyzed for amino acid content was also examined using PROC MIXED. Data points were excluded from analysis if they were both (1) outside of the range of serum amino acids found in horses based on previous literature and (2) more than three standard deviations away from the sample mean. Time between supplementation and BC2 was considered as a covariate but was not significant and, therefore, not included in the models. Horse, Treatment, and Day were recognized as class variables while Horse and Horse*Treatment were random effects. Day 1 and Day 3 data were considered separately using the SLICEBY=DAY statement. Treatment means were evaluated using the PDIFF option. Effect of treatment number (to evaluate the effectiveness of washout periods) and comparisons between blood collections and days were analyzed in PROC 
MIXED using the PDIFF option in LSMEANS. In these models, Horse, Treatment, TrtNum, Day, and blood collection were class variables while Horse and Horse*Treatment remained random effects.

The following models were used to evaluate serum amino acid content in Group 1:

$$
\begin{aligned}
& \text { TrpDiff }=\text { Treatment } \mid \text { Day } \\
& \text { RatioDiff }=\text { Treatment I Day }
\end{aligned}
$$

\section{RESULTS}

Horses remained healthy throughout the study; all resting heart rate, respiratory rate, body temperature, and packed cell volume measurements remained within normal limits. Individual horse body weight varied somewhat throughout the study and feed/treatment doses were adjusted accordingly. Serum glucose, lactate, cortisol, and amino acid levels are compared to expected ranges based on previous literature in Table 2.4.

Correlations between model covariates and response variables, along with covariate effects in the models, are presented in Table 2.5. Means and standard deviations for each response variable by treatment and day are displayed in Table 2.6.

$L O W$

While heart rate was elevated above baseline at 30 seconds post-startle for both the low dose and control on Day 1, heart rate difference was less on Day 1 of the low treatment compared to Day 1 of control $(P=0.05)$. Day 1 lactate difference was also significantly less on the low dose than on control $(P=0.03)$. On the low dose Day 1 , average serum lactate was less post startle test compared to values before the startle test. On control Day 1, however, lactate increased after the startle test. These results indicate that the low treatment had a sedative effect on Day 1 in terms of changes in heart rate and serum lactate levels.

No significant effects or trends were seen at the low treatment on Day 3. 
Day 1 cortisol difference on the medium treatment was less compared to control $(P=0.01)$. Serum cortisol decreased post-startle test on the medium dose while it increased post-startle on control. This seems to indicate that the medium treatment had a sedative effect on cortisol levels on Day 1.

Time for heart rate to return to baseline post-startle was greater on Day 3 of medium dose than control $(P=0.03)$. These results show that the medium dose had an excitatory effect on heart rate on Day 3.

$H I G H$

Cortisol difference before and after the startle test tended to be different on Day 1 of the high dose compared to control $(P=0.10)$. Numerically, serum cortisol levels were lower poststartle than pre-startle on high treatment Day 1 where levels actually increased after the startle test on control Day 1. This trend implies that the high dose may have had a sedative effect on cortisol levels on Day 1.

Post-startle serum lactate on Day 3 of the high dose showed a trend of being higher than on Day 3 of control $(P=0.10)$, suggesting somewhat of an excitatory effect on this response variable.

\section{Amino Acids}

Mean differences in serum free tryptophan and Trp:LNAA by treatment and day are presented in Figures 2.2 and 2.3, respectively. Significance for pairwise comparisons of means between treatments are shown in Tables 2.7 and 2.8 .

On Day 1, serum Trp and Trp:LNAA in BC1 did differ significantly from levels in BC2 ( $P$ $<0.0001$ and $P<0.0001$, respectively). However, on Day 3, neither Trp nor Trp:LNAA differed 
significantly from $\mathrm{BC} 1$ to $\mathrm{BC} 2(P=0.98$ and $P=0.99$, respectively). In addition, Trp and Trp:LNAA on Day 3 BC1 were not significantly different from those seen on Day 1 BC2 $(P=$ 0.93 and $P=0.87$, respectively).

The efficacy of the washout periods was analyzed by comparing Trp and TRP:LNAA in BC1 on Day 1 of each treatment. Treatment number was not significant for serum Trp levels $(P=$ $0.54)$ or Trp:LNAA $(P=0.37)$.

\section{DISCUSSION}

We did see serum cortisol levels higher than those previously reported in horses. Serum free tryptophan was also seen at higher concentrations than those previously reported but we provided oral tryptophan supplement to horses at doses that have not been previously evaluated.

It is interesting to note that on Day 1, all treatments had some sort of sedative effect on at least one of the response variables whereas on Day 3, either no effect was seen (LOW) or some sort of excitatory effect was seen (MED, $\mathrm{HIGH})$ on one of the response variables. The reason for this cannot be determined based on this experiment, but one possibility is that horses started to experience symptoms of serotonin syndrome and became more excitable on Day 3 at the MED and HIGH doses. However, we did not see any of the symptoms of the syndrome as they present in other species (Boyer and Shannon, 2005; Gillman, 1999).

Another potential explanation for the results on Day 3 conflicting with those seen on Day 1 could be that it is the actual change in the Trp:LNAA ratio that produces the desired sedative behavioral effects noted in other species. Some evidence for this may lie in the serum amino acid analysis of Group 1 horses, as treatment on Day 3 had little effect on serum free tryptophan concentration or Trp:LNAA. The lack of significant changes in amino acid profiles on Day 3 and from Day 1 to Day 3 may provide reasoning as to why no significant calming effects were seen in 
any of the response variables on Day 3. It is possible that serum amino acids would have dropped between the time of $\mathrm{BC} 1$ and $\mathrm{BC} 2$ on Day 3, had the horses not received another treatment during that time. Again, we are unsure as to why this is; without further amino acid analysis, we do not know how long free tryptophan or Trp:LNAA remain elevated in the serum. We can see that after the four-day washout period, levels returned to baseline and treatment number had no significant effect.

We were surprised at how little significance treatment had on most of the response variables and how trends did not seem to be reliable between treatments or days. One exception can be seen in the decrease in serum cortisol post-startle on Day 1 of the MED and HIGH treatments. These results agree with the response Koopmans et al. (2005) saw in swine supplemented with tryptophan; although no behavioral effects were seen in that study, tryptophan supplementation reduced plasma cortisol and noradrenaline in pigs exposed to social stress.

There was a lot of variability among individual horses used in this study. Perhaps testing a more homogenous group of horses (in which individuals were of the same breed, sex, and similar age) would generate more clear results. Previous research provides evidence that there are differences in male and female serotonergic systems and responses to changes in dietary Trp:LNAA (Albonetti et al., 1994; Dickinson and Curzon, 1986; Henry et al., 1992; Henry et al., 1996; Kennett et al., 1986; Kim and Camilleri, 2000; Rouvinen et al., 1999). Alberghina et al. (2014) as well as Ferlazzo et al. (2012) saw differences in serum tryptophan in horses of different ages. Other studies have observed breed differences (Alberghina et al., 2014; Bagshaw et al., 1994; Grimmett and Sillence, 2005). While all of these factors may play a role in the permeability of the blood-brain barrier the effectiveness of supplemental tryptophan on serotonin biosynthesis, it is also worth considering that these types of treatments may be most effective in horses with 
dysfunctioning serotonergic systems. This would be in line with research in other species, where tryptophan supplements and proserotonergic drugs were most effective in individuals that exhibited symptoms of disorder within the serotonin system (Argyropoulos et al., 2004; Bell et al., 2001; Weld et al., 1998).

The environment was another source of variability. Although researchers aimed to keep the timing of the tests and the footing within the test chute as consistent as possible, variations in temperature, wind, precipitation, and on-farm activities provided uncontrolled stimuli that may have impacted the results of the behavior tests. Performing these tests in a more controlled environment would be desirable and may provide more reliable results.

Including a positive control, with a sedative known to be effective in horses (such as acepromazine), may have helped elucidate treatment effects. It's possible that the startling stimuli were too effective at spooking the horses in this experiment and that any treatment effects were nullified as a result. Comparing treatment effects to that of a positive control could provide support or evidence against this hypothesis.

Supplementing tryptophan to horses at higher doses $(40 \mathrm{mg} / \mathrm{kg} \mathrm{BW}$ and $60 \mathrm{mg} / \mathrm{kg} \mathrm{BW})$ may be an effective way to reduce cortisol levels in stressful situations. We also saw some evidence of calming effects of tryptophan at a lower dose $(20 \mathrm{mg} / \mathrm{kg} \mathrm{BW})$. However, these findings were seen in only a few of the results variables measured in this experiment. The horses we used responded to tryptophan supplementation more favorably on the first day they received the supplement. This finding does not support the directions found on some commercially available products that recommend providing the supplement to the horse 24 hours in advance and again several hours before a stressful event takes place. The subset of blood samples we analyzed for amino acid content provides evidence to refute this recommendation as well. We saw no additional 
beneficial effects in terms of increasing serum free tryptophan levels or the ratio of tryptophan to other large neutral amino acids on Day 3 of supplementation versus Day 1. This experiment confirms that supplementing horses with tryptophan is effective at increasing serum free tryptophan and the ratio of tryptophan to certain amino acids. We found little evidence for the behavioral or physiological calmative effects of supplemental tryptophan in horses at the doses tested. However, from the results presented and discussed in this paper, it appears that supplementing horses with tryptophan may produce desired results only a few hours after administration and that longer term use may provide no additional benefit or may even have unwanted effects.

When evaluating the use of calming supplements or drugs, it's important to consider the welfare of the horse. While these compounds may be beneficial in alleviating short-term stress and anxiety (for example, when veterinary care needs to be provided) the cause of such emotions should be evaluated. Horses kept in unnatural environments, managed poorly, or asked to perform beyond their level of training may show signs of stress and anxiety. Chronic health issues such as ulcers or lameness may also be the culprit. Oftentimes, sedative drugs and supplements are utilized to limit unwanted behaviors such as spooking, bolting, rearing, or bucking. Looking into the potential causes of unwanted behaviors should be the first step before owners turn to calming drugs or supplements. Providing more training, turnout time, or treatment for an underlying disease or condition could result in a more sustainable way to reduce a horse's unwanted behaviors and could improve welfare for the animal. 
TABLE 2.1: Horse profile by group

\begin{tabular}{|c|c|c|c|c|c|c|c|}
\hline & $n$ & $\begin{array}{c}\text { Mean } \\
\text { age (years) }\end{array}$ & $\begin{array}{c}\text { Mean } \\
\text { body } \\
\text { weight }(\mathrm{kg})\end{array}$ & $\begin{array}{c}\text { Mean } \\
\text { BCS (1-9) }\end{array}$ & $\begin{array}{c}\text { Mean } \\
\text { height }(\mathrm{cm})\end{array}$ & $\begin{array}{l}\text { Number of } \\
\text { mares: } \\
\text { Number of } \\
\text { geldings }\end{array}$ & $\begin{array}{c}\text { Number of } \\
\text { Quarter } \\
\text { Horse type: } \\
\text { Number of } \\
\text { draft horse } \\
\text { type }\end{array}$ \\
\hline Group 1 & 4 & 6.5 & 463.0 & 4.4 & 152.8 & $0: 4$ & $3: 1$ \\
\hline Group 2 & 3 & 8.6 & 493.9 & 5.7 & 160.3 & $0: 3$ & $0: 3$ \\
\hline Group 3 & 4 & 11.0 & 618 & 5.8 & 162.8 & $2: 4$ & $0: 4$ \\
\hline
\end{tabular}


TABLE 2.2: Variance between horses

\begin{tabular}{|c|c|c|c|c|c|c|}
\hline \multirow[b]{2}{*}{ Response Variable } & \multicolumn{3}{|c|}{ Day 1} & \multicolumn{3}{|c|}{ Day 3} \\
\hline & Estimate & Standard Error & $\begin{array}{c}\text { Ratio } \\
\text { (horse : residual) }\end{array}$ & Estimate & Standard Error & $\begin{array}{c}\text { Ratio } \\
\text { (horse : residual) }\end{array}$ \\
\hline $\begin{array}{l}\text { ExitSpeed } \\
(\mathrm{m} / \mathrm{s})\end{array}$ & 0.953 & 0.5521 & 1.773 & 0.04921 & 0.2089 & 0.03719 \\
\hline $\begin{array}{l}\mathrm{HR} 30 \\
\text { (bpm) }\end{array}$ & 215.49 & 131.02 & 0.7418 & 208.61 & 144.05 & 0.5164 \\
\hline $\begin{array}{l}\text { HR_Diff } \\
\text { (bpm) }\end{array}$ & 190.12 & 131.37 & 0.7159 & 34.1998 & 81.1269 & 0.07998 \\
\hline $\begin{array}{l}\text { TimetoBL } \\
(\mathrm{sec})\end{array}$ & 732.31 & 1142.78 & 0.1449 & 7143.96 & 4253.96 & 0.8022 \\
\hline $\begin{array}{l}\text { GluDiff } \\
(\mathrm{mg} / \mathrm{dL})\end{array}$ & 8.6686 & 7.4146 & 0.4094 & 0 & . & 0 \\
\hline $\begin{array}{l}\text { GluPostAvg } \\
(\mathrm{mg} / \mathrm{dL})\end{array}$ & 0 & . & 0 & 2.0242 & 9.4039 & 0.03188 \\
\hline $\begin{array}{l}\text { LactDiff } \\
(\mathrm{mmol} / \mathrm{L})\end{array}$ & 0.0113 & 0.01392 & 0.1805 & 0.01986 & 0.01978 & 0.2628 \\
\hline $\begin{array}{l}\text { LactPostAvg } \\
(\mathrm{mmol} / \mathrm{L})\end{array}$ & 0.02363 & 0.01927 & 0.4152 & 0.02802 & 0.02414 & 0.3843 \\
\hline $\begin{array}{l}\text { CortDiff } \\
(\mu \mathrm{g} / \mathrm{dL})\end{array}$ & 0 & . & 0 & 1.046 & 3.3003 & 0.06633 \\
\hline $\begin{array}{l}\text { CortPostAvg } \\
(\mu \mathrm{g} / \mathrm{dL})\end{array}$ & 87.7944 & 41.2961 & 5.5207 & 48.3134 & 24.9931 & 2.2064 \\
\hline
\end{tabular}




\section{TABLE 2.3: Guaranteed analysis of feedstuffs on as-fed basis}

\begin{tabular}{|c|c|c|}
\hline & $\begin{array}{c}\text { Hay: } \\
\text { Timothy Brome Mix }\end{array}$ & $\begin{array}{c}\text { Concentrate: } \\
\text { Purina Equine Senior }\end{array}$ \\
\hline Digestible Energy (Mcal/kg) & 2.07 & 2.70 \\
\hline Dry Matter (\%) & 92.3 & 90.0 \\
\hline Crude Protein $(\%)$ & 6.70 & $\min 14$ \\
\hline Estimated Lysine (\%) & 0.23 & $\min 0.70$ \\
\hline Acid Detergent Fiber (\%) & 33.5 & $*$ \\
\hline Neutral Detergent Fiber (\%) & 55.5 & $*$ \\
\hline Starch $(\%)$ & 0.2 & $\max 12$ \\
\hline Crude Fat $(\%)$ & 2.2 & $\min 5.5$ \\
\hline Ash (\%) & 4.9 & $*$ \\
\hline Calcium (\%) & 0.41 & $0.5-1.00$ \\
\hline Phosphorus (\%) & 0.17 & $\min 0.4$ \\
\hline Magnesium (\%) & 0.17 & 0.33 \\
\hline Potassium (\%) & 1.12 & $\min 1.60$ \\
\hline Sodium $(\%)$ & 0.03 & $\min 0.24$ \\
\hline Iron (ppm) & 64 & $\min 220$ \\
\hline Zinc (ppm) & 14 & $\min 220$ \\
\hline Copper (ppm) & 4 & $\min 55$ \\
\hline Manganese (ppm) & 44 & $\min 220$ \\
\hline
\end{tabular}


TABLE 2.4: Daily procedures

\begin{tabular}{|c|c|c|c|}
\hline Day & Group A & Group B & Group C \\
\hline 1 & $\begin{array}{l}\text { Heart rate, temperature, weigh, null } \\
\text { reactivity test }\end{array}$ & & \\
\hline 2 & $\begin{array}{l}\text { Heart rate, temperature, null reactivity } \\
\text { test }\end{array}$ & $\begin{array}{l}\text { Heart rate, temperature, weigh, null } \\
\text { reactivity test }\end{array}$ & \\
\hline 3 & $\begin{array}{l}\text { Heart rate, temperature, weigh, null } \\
\text { reactivity test }\end{array}$ & $\begin{array}{l}\text { Heart rate, temperature, null reactivity } \\
\text { test }\end{array}$ & \\
\hline 4 & $\begin{array}{l}\text { Heart rate, temperature, null reactivity } \\
\text { test }\end{array}$ & $\begin{array}{l}\text { Heart rate, temperature, weigh, null } \\
\text { reactivity test }\end{array}$ & \\
\hline 5 & $\begin{array}{l}\text { Heart rate, temperature, weigh, null } \\
\text { reactivity test }\end{array}$ & $\begin{array}{l}\text { Heart rate, temperature, null reactivity } \\
\text { test }\end{array}$ & $\begin{array}{l}\text { Heart rate, temperature, weigh, null } \\
\text { reactivity test }\end{array}$ \\
\hline 6 & $\begin{array}{l}\text { Heart rate, temperature, null reactivity } \\
\text { test }\end{array}$ & $\begin{array}{l}\text { Heart rate, temperature, weigh, null } \\
\text { reactivity test }\end{array}$ & $\begin{array}{l}\text { Heart rate, temperature, null reactivity } \\
\text { test }\end{array}$ \\
\hline 7 & $\begin{array}{l}\text { Heart rate, temperature, } \mathrm{BC} 1 \text { (PCV, } \\
\text { TRP:LNAA), weigh, } \mathrm{BC} 2 \text { (AA, glucose, } \\
\text { lactate, cortisol), null reactivity test, } \mathrm{BC} 3 \\
\text { (glucose, lactate, cortisol) }\end{array}$ & $\begin{array}{l}\text { Heart rate, temperature, null reactivity } \\
\text { test }\end{array}$ & $\begin{array}{l}\text { Heart rate, temperature, weigh, null } \\
\text { reactivity test }\end{array}$ \\
\hline 8 & $\begin{array}{l}\mathrm{BC} 1 \text { (PCV, AA), weigh, begin first* } \\
\text { treatment, heart rate, temperature, BC2 } \\
\text { (AA, glucose, lactate, cortisol), startle } \\
\text { reactivity test, BC3 (glucose, lactate, } \\
\text { cortisol) }\end{array}$ & $\begin{array}{l}\text { Heart rate, temperature, BC1 (PCV, } \\
\text { TRP:LNAA), weigh, BC2 (AA, glucose, } \\
\text { lactate, cortisol), null reactivity test, BC3 } \\
\text { (glucose, lactate, cortisol) }\end{array}$ & $\begin{array}{l}\text { Heart rate, temperature, null reactivity } \\
\text { test }\end{array}$ \\
\hline 9 & $\begin{array}{l}\mathrm{BC} 1(\mathrm{PCV}, \mathrm{AA}) \text {, treatment, heart rate, } \\
\text { temperature, } \mathrm{BC} 2(\mathrm{AA}) \text {, null reactivity } \\
\text { test }\end{array}$ & $\begin{array}{l}\mathrm{BC} 1 \text { (PCV, AA), weigh, begin first* } \\
\text { treatment, heart rate, temperature, BC2 } \\
\text { (AA, glucose, lactate, cortisol), startle } \\
\text { reactivity test, BC3 (glucose, lactate, } \\
\text { cortisol) }\end{array}$ & $\begin{array}{l}\text { Heart rate, temperature, weigh, null } \\
\text { reactivity test }\end{array}$ \\
\hline
\end{tabular}




\begin{tabular}{|c|c|c|c|}
\hline 10 & $\begin{array}{l}\mathrm{BC} 1 \text { (PCV, } \mathrm{AA}) \text {, treatment, heart rate, } \\
\text { temperature, } \mathrm{BC} 2 \text { (AA, glucose, lactate, } \\
\text { cortisol), startle reactivity test, } \mathrm{BC} 3 \\
\text { (glucose, lactate, cortisol) }\end{array}$ & $\begin{array}{l}\mathrm{BC} 1(\mathrm{PCV}, \mathrm{AA}) \text {, treatment, heart rate, } \\
\text { temperature, } \mathrm{BC} 2(\mathrm{AA}) \text {, null reactivity } \\
\text { test }\end{array}$ & $\begin{array}{l}\text { Heart rate, temperature, null reactivity } \\
\text { test }\end{array}$ \\
\hline 11 & $\begin{array}{l}\text { Washout day: heart rate, temperature, } \\
\text { BC1 (PCV), null reactivity test }\end{array}$ & $\begin{array}{l}\mathrm{BC} 1 \text { (PCV, } \mathrm{AA}) \text {, treatment, heart rate, } \\
\text { temperature, } \mathrm{BC} 2 \text { (AA, glucose, lactate, } \\
\text { cortisol), startle reactivity test, } \mathrm{BC} 3 \\
\text { (glucose, lactate, cortisol) }\end{array}$ & $\begin{array}{l}\text { Heart rate, temperature, BC1 (PCV, } \\
\text { TRP:LNAA), weigh, BC2 (AA, glucose, } \\
\text { lactate, cortisol), null reactivity test, } \mathrm{BC} 3 \\
\text { (glucose, lactate, cortisol) }\end{array}$ \\
\hline 12 & $\begin{array}{l}\text { Washout day: heart rate, temperature, } \\
\text { BC1 (PCV), null reactivity test }\end{array}$ & $\begin{array}{l}\text { Washout day: heart rate, temperature, } \\
\text { BC1 (PCV), null reactivity test }\end{array}$ & $\begin{array}{l}\mathrm{BC} 1 \text { (PCV, AA), weigh, begin first* } \\
\text { treatment, heart rate, temperature, } \mathrm{BC} 2 \\
\text { (AA, glucose, lactate, cortisol), startle } \\
\text { reactivity test, BC3 (glucose, lactate, } \\
\text { cortisol) }\end{array}$ \\
\hline 13 & $\begin{array}{l}\text { Washout day: heart rate, temperature, } \\
\text { BC1 (PCV), null reactivity test }\end{array}$ & $\begin{array}{l}\text { Washout day: heart rate, temperature, } \\
\text { BC1 (PCV), null reactivity test }\end{array}$ & $\begin{array}{l}\mathrm{BC} 1(\mathrm{PCV}, \mathrm{AA}) \text {, treatment, heart rate, } \\
\text { temperature, } \mathrm{BC} 2(\mathrm{AA}) \text {, null reactivity } \\
\text { test }\end{array}$ \\
\hline 14 & $\begin{array}{l}\text { Washout day: heart rate, temperature, } \\
\text { BC1 (PCV), null reactivity test }\end{array}$ & $\begin{array}{l}\text { Washout day: heart rate, temperature, } \\
\text { BC1 (PCV), null reactivity test }\end{array}$ & $\begin{array}{l}\mathrm{BC1}(\mathrm{PCV}, \mathrm{AA}) \text {, treatment, heart rate, } \\
\text { temperature, } \mathrm{BC} 2 \text { (AA, glucose, lactate, } \\
\text { cortisol), startle reactivity test, } \mathrm{BC} 3 \\
\text { (glucose, lactate, cortisol) }\end{array}$ \\
\hline 15 & $\begin{array}{l}\mathrm{BC} 1(\mathrm{PCV}, \mathrm{AA}) \text {, weigh, begin second* } \\
\text { treatment, heart rate, temperature, } \mathrm{BC} 2 \\
\text { (AA, glucose, lactate, cortisol), startle } \\
\text { reactivity test, } \mathrm{BC} 3 \text { (glucose, lactate, } \\
\text { cortisol) }\end{array}$ & $\begin{array}{l}\text { Washout day: heart rate, temperature, } \\
\text { BC1 (PCV), null reactivity test }\end{array}$ & $\begin{array}{l}\text { Washout day: heart rate, temperature, } \\
\text { BC1 (PCV), null reactivity test }\end{array}$ \\
\hline 16 & $\begin{array}{l}\mathrm{BC} 1(\mathrm{PCV}, \mathrm{AA}) \text {, treatment, heart rate, } \\
\text { temperature, } \mathrm{BC} 2(\mathrm{AA}) \text {, null reactivity } \\
\text { test }\end{array}$ & $\begin{array}{l}\mathrm{BC} 1 \text { (PCV, } \mathrm{AA}) \text {, weigh, begin second* } \\
\text { treatment, heart rate, temperature, } \mathrm{BC} 2 \\
\text { (AA, glucose, lactate, cortisol), startle } \\
\text { reactivity test, BC3 (glucose, lactate, } \\
\text { cortisol) }\end{array}$ & $\begin{array}{l}\text { Washout day: heart rate, temperature, } \\
\text { BC1 (PCV), null reactivity test }\end{array}$ \\
\hline
\end{tabular}




\begin{tabular}{|c|c|c|c|}
\hline 17 & $\begin{array}{l}\mathrm{BC} 1 \text { (PCV, } \mathrm{AA}) \text {, treatment, heart rate, } \\
\text { temperature, } \mathrm{BC} 2 \text { (AA, glucose, lactate, } \\
\text { cortisol), startle reactivity test, BC3 } \\
\text { (glucose, lactate, cortisol) }\end{array}$ & $\begin{array}{l}\mathrm{BC} 1(\mathrm{PCV}, \mathrm{AA}) \text {, treatment, heart rate, } \\
\text { temperature, } \mathrm{BC} 2 \text { (AA), null reactivity } \\
\text { test }\end{array}$ & $\begin{array}{l}\text { Washout day: heart rate, temperature, } \\
\text { BC1 (PCV), null reactivity test }\end{array}$ \\
\hline 18 & $\begin{array}{l}\text { Washout day: heart rate, temperature, } \\
\text { BC1 (PCV), null reactivity test }\end{array}$ & $\begin{array}{l}\mathrm{BC} 1 \text { (PCV, AA), treatment, heart rate, } \\
\text { temperature, BC2 (AA, glucose, lactate, } \\
\text { cortisol), startle reactivity test, BC3 } \\
\text { (glucose, lactate, cortisol) }\end{array}$ & $\begin{array}{l}\text { Washout day: heart rate, temperature, } \\
\text { BC1 (PCV), null reactivity test }\end{array}$ \\
\hline 19 & $\begin{array}{l}\text { Washout day: heart rate, temperature, } \\
\text { BC1 (PCV), null reactivity test }\end{array}$ & $\begin{array}{l}\text { Washout day: heart rate, temperature, } \\
\text { BC1 (PCV), null reactivity test }\end{array}$ & $\begin{array}{l}\mathrm{BC} 1 \text { (PCV, AA), weigh, begin second* } \\
\text { treatment, heart rate, temperature, } \mathrm{BC} 2 \\
\text { (AA, glucose, lactate, cortisol), startle } \\
\text { reactivity test, BC3 (glucose, lactate, } \\
\text { cortisol) }\end{array}$ \\
\hline 20 & $\begin{array}{l}\text { Washout day: heart rate, temperature, } \\
\text { BC1 (PCV), null reactivity test }\end{array}$ & $\begin{array}{l}\text { Washout day: heart rate, temperature, } \\
\text { BC1 (PCV), null reactivity test }\end{array}$ & $\begin{array}{l}\mathrm{BC} 1(\mathrm{PCV}, \mathrm{AA}) \text {, treatment, heart rate, } \\
\text { temperature, } \mathrm{BC} 2(\mathrm{AA}) \text {, null reactivity } \\
\text { test }\end{array}$ \\
\hline 21 & $\begin{array}{l}\text { Washout day: heart rate, temperature, } \\
\text { BC1 (PCV), null reactivity test }\end{array}$ & $\begin{array}{l}\text { Washout day: heart rate, temperature, } \\
\text { BC1 (PCV), null reactivity test }\end{array}$ & $\begin{array}{l}\mathrm{BC} 1 \text { (PCV, } \mathrm{AA}) \text {, treatment, heart rate, } \\
\text { temperature, } \mathrm{BC} 2 \text { (AA, glucose, lactate, } \\
\text { cortisol), startle reactivity test, BC3 } \\
\text { (glucose, lactate, cortisol) }\end{array}$ \\
\hline 22 & $\begin{array}{l}\mathrm{BC} 1 \text { (PCV, AA), weigh, begin third* } \\
\text { treatment, heart rate, temperature, BC2 } \\
\text { (AA, glucose, lactate, cortisol), startle } \\
\text { reactivity test, BC3 (glucose, lactate, } \\
\text { cortisol) }\end{array}$ & $\begin{array}{l}\text { Washout day: heart rate, temperature, } \\
\text { BC1 (PCV), null reactivity test }\end{array}$ & $\begin{array}{l}\text { Washout day: heart rate, temperature, } \\
\text { BC1 (PCV), null reactivity test }\end{array}$ \\
\hline
\end{tabular}




\begin{tabular}{|c|c|c|c|}
\hline 23 & $\begin{array}{l}\mathrm{BC} 1(\mathrm{PCV}, \mathrm{AA}) \text {, treatment, heart rate, } \\
\text { temperature, } \mathrm{BC} 2(\mathrm{AA}) \text {, null reactivity } \\
\text { test }\end{array}$ & $\begin{array}{l}\mathrm{BC} 1 \text { (PCV, AA), weigh, begin third* } \\
\text { treatment, heart rate, temperature, BC2 } \\
\text { (AA, glucose, lactate, cortisol), startle } \\
\text { reactivity test, BC3 (glucose, lactate, } \\
\text { cortisol) }\end{array}$ & $\begin{array}{l}\text { Washout day: heart rate, temperature, } \\
\text { BC1 (PCV), null reactivity test }\end{array}$ \\
\hline 24 & $\begin{array}{l}\mathrm{BC} 1 \text { (PCV, } \mathrm{AA}) \text {, treatment, heart rate, } \\
\text { temperature, } \mathrm{BC} 2 \text { (AA, glucose, lactate, } \\
\text { cortisol), startle reactivity test, BC3 } \\
\text { (glucose, lactate, cortisol) }\end{array}$ & $\begin{array}{l}\mathrm{BC} 1(\mathrm{PCV}, \mathrm{AA}) \text {, treatment, heart rate, } \\
\text { temperature, } \mathrm{BC} 2(\mathrm{AA}) \text {, null reactivity } \\
\text { test }\end{array}$ & $\begin{array}{l}\text { Washout day: heart rate, temperature, } \\
\text { BC1 (PCV), null reactivity test }\end{array}$ \\
\hline 25 & $\begin{array}{l}\text { Washout day: heart rate, temperature, } \\
\text { BC1 (PCV), null reactivity test }\end{array}$ & $\begin{array}{l}\mathrm{BC} 1 \text { (PCV, } \mathrm{AA}) \text {, treatment, heart rate, } \\
\text { temperature, } \mathrm{BC} 2 \text { (AA, glucose, lactate, } \\
\text { cortisol), startle reactivity test, } \mathrm{BC} 3 \\
\text { (glucose, lactate, cortisol) }\end{array}$ & $\begin{array}{l}\text { Washout day: heart rate, temperature, } \\
\text { BC1 (PCV), null reactivity test }\end{array}$ \\
\hline 26 & $\begin{array}{l}\text { Washout day: heart rate, temperature, } \\
\text { BC1 (PCV), null reactivity test }\end{array}$ & $\begin{array}{l}\text { Washout day: heart rate, temperature, } \\
\text { BC1 (PCV), null reactivity test }\end{array}$ & $\begin{array}{l}\mathrm{BC} 1 \text { (PCV, AA), weigh, begin third* } \\
\text { treatment, heart rate, temperature, BC2 } \\
\text { (AA, glucose, lactate, cortisol), startle } \\
\text { reactivity test, BC3 (glucose, lactate, } \\
\text { cortisol) }\end{array}$ \\
\hline
\end{tabular}




\begin{tabular}{|c|c|c|c|}
\hline 27 & $\begin{array}{l}\text { Washout day: heart rate, temperature, } \\
\text { BC1 (PCV), null reactivity test }\end{array}$ & $\begin{array}{l}\text { Washout day: heart rate, temperature, } \\
\text { BC1 (PCV), null reactivity test }\end{array}$ & $\begin{array}{l}\mathrm{BC} 1(\mathrm{PCV}, \mathrm{AA}) \text {, treatment, heart rate, } \\
\text { temperature, } \mathrm{BC} 2(\mathrm{AA}) \text {, null reactivity } \\
\text { test }\end{array}$ \\
\hline 28 & $\begin{array}{l}\text { Washout day: heart rate, temperature, } \\
\text { baseline blood draw (PCV), weigh, null } \\
\text { reactivity test }\end{array}$ & $\begin{array}{l}\text { Washout day: heart rate, temperature, } \\
\text { BC1 (PCV), null reactivity test }\end{array}$ & $\begin{array}{l}\mathrm{BC} 1 \text { (PCV, } \mathrm{AA}) \text {, treatment, heart rate, } \\
\text { temperature, } \mathrm{BC} 2 \text { (AA, glucose, lactate, } \\
\text { cortisol), startle reactivity test, } \mathrm{BC} 3 \\
\text { (glucose, lactate, cortisol) }\end{array}$ \\
\hline 29 & $\begin{array}{l}\mathrm{BC} 1 \text { (PCV, AA), weigh, begin fourth* } \\
\text { treatment, heart rate, temperature, BC2 } \\
\text { (AA, glucose, lactate, cortisol), startle } \\
\text { reactivity test, BC3 (glucose, lactate, } \\
\text { cortisol) }\end{array}$ & $\begin{array}{l}\text { Washout day: heart rate, temperature, } \\
\text { BC1 (PCV), null reactivity test }\end{array}$ & $\begin{array}{l}\text { Washout day: heart rate, temperature, } \\
\text { BC1 (PCV), null reactivity test }\end{array}$ \\
\hline 30 & $\begin{array}{l}\mathrm{BC} 1(\mathrm{PCV}, \mathrm{AA}) \text {, treatment, heart rate, } \\
\text { temperature, } \mathrm{BC} 2(\mathrm{AA}) \text {, null reactivity } \\
\text { test }\end{array}$ & $\begin{array}{l}\mathrm{BC} 1 \text { (PCV, AA), weigh, begin fourth* } \\
\text { treatment, heart rate, temperature, BC2 } \\
\text { (AA, glucose, lactate, cortisol), startle } \\
\text { reactivity test, BC3 (glucose, lactate, } \\
\text { cortisol) }\end{array}$ & $\begin{array}{l}\text { Washout day: heart rate, temperature, } \\
\text { BC1 (PCV), null reactivity test }\end{array}$ \\
\hline
\end{tabular}




\begin{tabular}{|c|c|c|c|}
\hline 31 & $\begin{array}{l}\mathrm{BC} 1 \text { (PCV, } \mathrm{AA} \text { ), treatment, heart rate, } \\
\text { temperature, } \mathrm{BC} 2 \text { (AA, glucose, lactate, } \\
\text { cortisol), startle reactivity test, } \mathrm{BC} 3 \\
\text { (glucose, lactate, cortisol) }\end{array}$ & $\begin{array}{l}\mathrm{BC} 1(\mathrm{PCV}, \mathrm{AA}) \text {, treatment, heart rate, } \\
\text { temperature, } \mathrm{BC} 2(\mathrm{AA}) \text {, null reactivity } \\
\text { test }\end{array}$ & $\begin{array}{l}\text { Washout day: heart rate, temperature, } \\
\text { BC1 (PCV), null reactivity test }\end{array}$ \\
\hline 32 & $\begin{array}{l}\text { Washout day: heart rate, temperature, } \\
\text { baseline blood draw (PCV), null } \\
\text { reactivity test }\end{array}$ & $\begin{array}{l}\mathrm{BC} 1(\mathrm{PCV}, \mathrm{AA}) \text {, treatment, heart rate, } \\
\text { temperature, } \mathrm{BC} 2 \text { (AA, glucose, lactate, } \\
\text { cortisol), startle reactivity test, } \mathrm{BC} 3 \\
\text { (glucose, lactate, cortisol) }\end{array}$ & $\begin{array}{l}\text { Washout day: heart rate, temperature, } \\
\text { BC1 (PCV), null reactivity test }\end{array}$ \\
\hline 33 & $\begin{array}{l}\text { Washout day: heart rate, temperature, } \\
\text { baseline blood draw (PCV), null } \\
\text { reactivity test }\end{array}$ & $\begin{array}{l}\text { Washout day: heart rate, temperature, } \\
\text { BC1 (PCV), null reactivity test }\end{array}$ & $\begin{array}{l}\mathrm{BC} 1 \text { (PCV, } \mathrm{AA}) \text {, weigh, begin fourth* } \\
\text { treatment, heart rate, temperature, BC2 } \\
\text { (AA, glucose, lactate, cortisol), startle } \\
\text { reactivity test, BC3 (glucose, lactate, } \\
\text { cortisol) }\end{array}$ \\
\hline 34 & $\begin{array}{l}\text { Washout day: heart rate, temperature, } \\
\text { baseline blood draw (PCV), null } \\
\text { reactivity test }\end{array}$ & $\begin{array}{l}\text { Washout day: heart rate, temperature, } \\
\text { BC1 (PCV), null reactivity test }\end{array}$ & $\begin{array}{l}\mathrm{BC} 1(\mathrm{PCV}, \mathrm{AA}) \text {, treatment, heart rate, } \\
\text { temperature, } \mathrm{BC} 2(\mathrm{AA}) \text {, null reactivity } \\
\text { test }\end{array}$ \\
\hline
\end{tabular}




\begin{tabular}{|l|l|l|l|}
\hline 35 & $\begin{array}{l}\text { Washout day: heart rate, temperature, } \\
\text { baseline blood draw (PCV), weigh, null } \\
\text { reactivity test }\end{array}$ & $\begin{array}{l}\text { Washout day: heart rate, temperature, } \\
\mathrm{BC1}(\mathrm{PCV}), \text { null reactivity test }\end{array}$ & $\begin{array}{l}\mathrm{BC} 1 \text { (PCV, } \mathrm{AA}), \text { treatment, heart rate, } \\
\text { temperature, BC2 (AA, glucose, lactate, } \\
\text { (glucose, lactate, cortisol) }\end{array}$ \\
\hline 36 & & $\begin{array}{l}\text { Washout day: heart rate, temperature, } \\
\text { BC1 (PCV), null reactivity test }\end{array}$ & $\begin{array}{l}\text { Washout day: heart rate, temperature, } \\
\text { BC1 (PCV), null reactivity test }\end{array}$ \\
\hline 37 & & & $\begin{array}{l}\text { Washout day: heart rate, temperature, } \\
\text { BC1 (PCV), null reactivity test }\end{array}$ \\
\hline 38 & & & $\begin{array}{l}\text { Washout day: heart rate, temperature, } \\
\text { BC1 (PCV), null reactivity test }\end{array}$ \\
\hline 39 & & & $\begin{array}{l}\text { Washout day: heart rate, temperature, } \\
\text { BC1 (PCV), null reactivity test }\end{array}$ \\
\hline
\end{tabular}

*Fixed treatments (CON, LOW, MED, HIGH) were assigned in a random order so that no horses within a group were receiving the same treatment at the same time 


\section{TABLE 2.5: Serum values in horses}

\begin{tabular}{|c|c|c|}
\hline Compound & Ranges found in previous literature & Ranges found in current study \\
\hline Glucose (mg/dL) & $62^{1}-134^{1}$ & $67.5-112.5$ \\
\hline Lactate $(\mathrm{mmol} / \mathrm{L})$ & $0.56^{2}-2.32^{3}$ & $0.47-2.78$ \\
\hline Cortisol ( $\mu \mathrm{g} / \mathrm{dL})$ & $0.08^{4}-25.5^{4}$ & $0.02-56.81$ \\
\hline Free tryptophan $(\mu \mathrm{mol} / \mathrm{L})$ & $8.0^{5}-118.1^{6}$ & $19.3-142.3$ \\
\hline Isoleucine $(\mu \mathrm{mol} / \mathrm{L})$ & $36.1^{5}-171.8^{5}$ & $72.3-120.1$ \\
\hline Leucine $(\mu \mathrm{mol} / \mathrm{L})$ & $60.1^{6}-371.3^{5}$ & $122.4-148.2$ \\
\hline Valine $(\mu \mathrm{mol} / \mathrm{L})$ & $115.0^{5}-311.8^{5}$ & $164.1-201.3$ \\
\hline Penylalanine $(\mu \mathrm{mol} / \mathrm{L})$ & $14.7^{6}-103.4^{6}$ & $31.1-57.3$ \\
\hline Tyrosine $(\mu \mathrm{mol} / \mathrm{L})$ & $22.1^{6}-125.4^{6}$ & $68.6-88.2$ \\
\hline Trp:LNAA & $0.09^{7}-0.13^{7}$ & $0.04-0.28$ \\
\hline $\begin{array}{l}{ }^{1} \text { Kahn and Line, } 2010 \\
{ }^{2} \text { Stull and Rodiek, } 2000 \\
{ }^{3} \text { Nogueira et al., } 2002 \\
{ }^{4} \text { Alenka et al., 2008 } \\
{ }^{5} \text { Assenza et al., 2004 } \\
{ }^{6} \text { Bergero et al., 2005 } \\
{ }^{7} \text { Wilson, 2007 }\end{array}$ & & \\
\hline
\end{tabular}


TABLE 2.6: Model covariates-correlation to response variables and influence in models

\begin{tabular}{|c|c|c|c|c|c|c|c|}
\hline \multirow{2}{*}{ Response Variable } & \multirow{2}{*}{ Covariate in Model } & \multicolumn{3}{|c|}{ DAY 1} & \multicolumn{3}{|c|}{ DAY 3} \\
\hline & & $\mathrm{r}^{*}$ & $P^{* *}$ & estimate $\pm \mathrm{SE}^{* * *}$ & $\mathrm{r}^{*}$ & $P^{* *}$ & estimate $\pm \mathrm{SE}^{* * *}$ \\
\hline \multicolumn{8}{|l|}{$\overline{\text { ExitSpeed }(\mathrm{m} / \mathrm{s})}$} \\
\hline & Group & -0.42097 & 0.0068 & & -0.44902 & 0.0022 & \\
\hline & Temperature $\left({ }^{\circ} \mathrm{C}\right)$ & 0.54964 & 0.0002 & $0.080 \pm 0.017$ & 0.11043 & 0.4755 & $0.002 \pm 0.029$ \\
\hline \multicolumn{8}{|l|}{ HR30 (bpm) } \\
\hline & TrtNum & 0.03286 & 0.8363 & & 0.31953 & 0.0391 & \\
\hline \multicolumn{8}{|l|}{ HR_Diff (bpm) } \\
\hline & Group & -0.31893 & 0.0395 & & -0.42359 & 0.0052 & \\
\hline & TrtNum & 0.12101 & 0.4452 & & 0.42941 & 0.0045 & \\
\hline \multicolumn{8}{|l|}{ TimetoBL (sec) } \\
\hline & Temperature $\left({ }^{\circ} \mathrm{C}\right)$ & 0.39175 & 0.0136 & $4.114 \pm 1.586$ & 0.46337 & 0.0023 & $10.950 \pm 2.523$ \\
\hline \multicolumn{8}{|l|}{ GluDiff (mg/dL) } \\
\hline & GluRunDate & -0.06339 & 0.7014 & & -0.38298 & 0.0161 & \\
\hline & TimeLag (sec) & -0.43873 & 0.0052 & $-0.048 \pm 0.032$ & -0.42515 & 0.0070 & $-0.105 \pm 0.032$ \\
\hline \multicolumn{8}{|c|}{ GluPostAvg (mg/dL) } \\
\hline & TimeLag (sec) & -0.38014 & 0.0142 & $-0.074 \pm 0.029$ & -0.39329 & 0.0110 & $-0.106 \pm 0.044$ \\
\hline \multicolumn{8}{|c|}{ CortPost Avg $(\mu \mathrm{g} / \mathrm{dL})$} \\
\hline & CortPosttestPlate & 0.13747 & 0.3914 & & 0.31034 & 0.0483 & \\
\hline
\end{tabular}

*PROC CORR: Pearson correlation coefficient

$* *$ PROC CORR: Prob > |r| under H0: Rho $=0$

***PROC MIXED: Solution for Fixed Effects 
TABLE 2.7: Effect of tryptophan treatment on physiological and behavioral response variables: least squares means \pm standard error

\begin{tabular}{|c|c|c|c|c|c|c|c|c|}
\hline \multirow[b]{2}{*}{ Response Variable } & \multicolumn{2}{|c|}{$\mathrm{CON}$} & \multicolumn{2}{|c|}{ LOW } & \multicolumn{2}{|c|}{ MED } & \multicolumn{2}{|c|}{ HIGH } \\
\hline & Day 1 & Day 3 & Day 1 & Day 3 & Day 1 & Day 3 & Day 1 & Day 3 \\
\hline ExitSpeed (m/s) & $5.09 \pm 0.38$ & $5.19 \pm 0.36$ & $4.77 \pm 0.38$ & $5.54 \pm 0.36$ & $5.16 \pm 0.37$ & $5.12 \pm 0.36$ & $5.44 \pm 0.39$ & $5.59 \pm 0.35$ \\
\hline HR30 (bpm) & $102.83 \pm 6.79$ & $108.36 \pm 7.48$ & $97.60 \pm 7.05$ & $103.66 \pm 7.48$ & $104.04 \pm 7.06$ & $99.91 \pm 7.80$ & $109.78 \pm 6.80$ & $99.02 \pm 7.80$ \\
\hline HR_Diff (bpm) & $69.95 \pm 6.48$ & $71.87 \pm 6.52$ & $55.33 \pm 6.72$ & $68.56 \pm 6.53$ & $68.79 \pm 6.77$ & $68.19 \pm 6.94$ & $74.51 \pm 6.48$ & $65.29 \pm 6.94$ \\
\hline TimetoBL (sec) & $224.00 \pm 24.15$ & $228.69 \pm 38.21$ & $213.53 \pm 25.51$ & $224.28 \pm 39.69$ & $249.62 \pm 24.14$ & $325.88 \pm 39.58$ & $209.59 \pm 24.19$ & $247.11 \pm 39.67$ \\
\hline GluDiff (mg/dL) & $-4.34 \pm 1.96$ & $-1.78 \pm 1.74$ & $-8.27 \pm 1.97$ & $1.79 \pm 1.69$ & $-4.96 \pm 2.07$ & $1.98 \pm 1.76$ & $-0.64 \pm 2.03$ & $0.54 \pm 1.90$ \\
\hline GluPostAvg (mg/dL) & $83.36 \pm 2.33$ & $85.17 \pm 2.57$ & $81.71 \pm 2.20$ & $85.41 \pm 2.45$ & $83.89 \pm 2.25$ & $86.90 \pm 2.44$ & $87.88 \pm 2.43$ & $87.00 \pm 2.73$ \\
\hline LactDiff (mmo/L) & $0.17 \pm 0.09$ & $0.17 \pm 0.10$ & $-0.10 \pm 0.09$ & $0.30 \pm 0.10$ & $0.21 \pm 0.08$ & $0.24 \pm 0.10$ & $0.22 \pm 0.09$ & $0.19 \pm 0.11$ \\
\hline LactPostAvg (mmol/L) & $1.14 \pm 0.09$ & $1.09 \pm 0.11$ & $0.98 \pm 0.09$ & $1.18 \pm 0.10$ & $1.25 \pm 0.09$ & $1.25 \pm 0.11$ & $1.11 \pm 0.09$ & $1.35 \pm 0.11$ \\
\hline CortDiff $(\mu \mathrm{g} / \mathrm{dL})$ & $2.79 \pm 1.27$ & $-0.29 \pm 1.30$ & $1.09 \pm 1.27$ & $0.33 \pm 1.24$ & $-1.86 \pm 1.22$ & $-0.07 \pm 1.30$ & $-0.38 \pm 1.34$ & $-0.38 \pm 1.38$ \\
\hline
\end{tabular}


TABLE 2.8: Differences of least squares means for serum free tryptophan (

\begin{tabular}{cc|r}
\multicolumn{3}{c}{ DAY 1 } \\
\hline \multicolumn{2}{c}{ Treatment } & $P^{*}$ \\
\hline $\mathrm{CON}^{\mathrm{a}}$ & LOW $^{\mathrm{b}}$ & 0.0011 \\
$\mathrm{CON}^{\mathrm{a}}$ & $\mathrm{MED}^{\mathrm{a}}$ & $<0.0001$ \\
$\mathrm{CON}^{\mathrm{a}}$ & $\mathrm{HIGH}^{\mathrm{a}}$ & $<0.0001$ \\
LOW $^{\mathrm{b}}$ & $\mathrm{MED}^{\mathrm{a}}$ & 0.0474 \\
LOW $^{\mathrm{b}}$ & $\mathrm{HIGH}^{\mathrm{a}}$ & $<0.0001$ \\
MED $^{\mathrm{a}}$ & $\mathrm{HIGH}^{\mathrm{a}}$ & 0.0013
\end{tabular}

DAY 3

\begin{tabular}{cc|r}
\hline \multicolumn{2}{c|}{ Treatment } & $P^{*}$ \\
\hline $\mathrm{CON}^{\mathrm{a}}$ & LOW $^{\mathrm{a}}$ & 0.7150 \\
$\mathrm{CON}^{\mathrm{a}}$ & MED $^{\mathrm{a}}$ & 0.5878 \\
$\mathrm{CON}^{\mathrm{a}}$ & $\mathrm{HIGH}^{\mathrm{b}}$ & 0.6035 \\
$\mathrm{LOW}^{\mathrm{a}}$ & $\mathrm{MED}^{\mathrm{a}}$ & 0.3675 \\
LOW $^{\mathrm{a}}$ & $\mathrm{HIGH}^{\mathrm{b}}$ & 0.8554 \\
MED $^{\mathrm{a}}$ & HIGH $^{\mathrm{b}}$ & 0.3114
\end{tabular}

${ }_{n=4}^{\mathrm{a}} .{ }^{\mathrm{b}} n=3 . *$ Difference of Least Squares Means.

TABLE 2.9: Differences of least squares means for Trp:LNAA Group 1 horses

\begin{tabular}{|c|c|c|c|c|c|}
\hline \multicolumn{3}{|c|}{ DAY 1} & \multicolumn{3}{|c|}{ DAY 3} \\
\hline \multicolumn{2}{|c|}{ Treatment } & \multirow{2}{*}{$\frac{P^{*}}{0.0037}$} & \multicolumn{2}{|c|}{ Treatment } & \multirow{2}{*}{$\frac{P^{*}}{0.5465}$} \\
\hline $\mathrm{CON}^{\mathrm{b}}$ & LOW $^{\mathrm{b}}$ & & $\mathrm{CON}^{\mathrm{b}}$ & $\mathrm{LOW}^{\mathrm{a}}$ & \\
\hline $\mathrm{CON}^{\mathrm{b}}$ & $\mathrm{MED}^{\mathrm{a}}$ & $<0.0001$ & $\mathrm{CON}^{\mathrm{b}}$ & $\mathrm{MED}^{\mathrm{a}}$ & 0.6022 \\
\hline $\mathrm{CON}^{\mathrm{b}}$ & $\mathrm{HIGH}^{\mathrm{b}}$ & $<0.0001$ & $\mathrm{CON}^{\mathrm{b}}$ & $\mathrm{HIGH}^{\mathrm{c}}$ & 0.9097 \\
\hline LOW $^{\mathrm{b}}$ & $\mathrm{MED}^{\mathrm{a}}$ & 0.0632 & $\mathrm{LOW}^{\mathrm{a}}$ & $\mathrm{MED}^{\mathrm{a}}$ & 0.2320 \\
\hline LOW $^{\mathrm{b}}$ & $\mathrm{HIGH}^{\mathrm{b}}$ & 0.0006 & $\mathrm{LOW}^{\mathrm{a}}$ & $\mathrm{HIGH}^{\mathrm{c}}$ & 0.6789 \\
\hline $\mathrm{MED}^{\mathrm{a}}$ & $\mathrm{HIGH}^{\mathrm{b}}$ & 0.0252 & $\mathrm{MED}^{\mathrm{a}}$ & $\mathrm{HIGH}^{\mathrm{c}}$ & 0.5631 \\
\hline
\end{tabular}


Start Timers

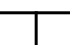

Blind

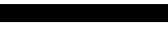

Release Point

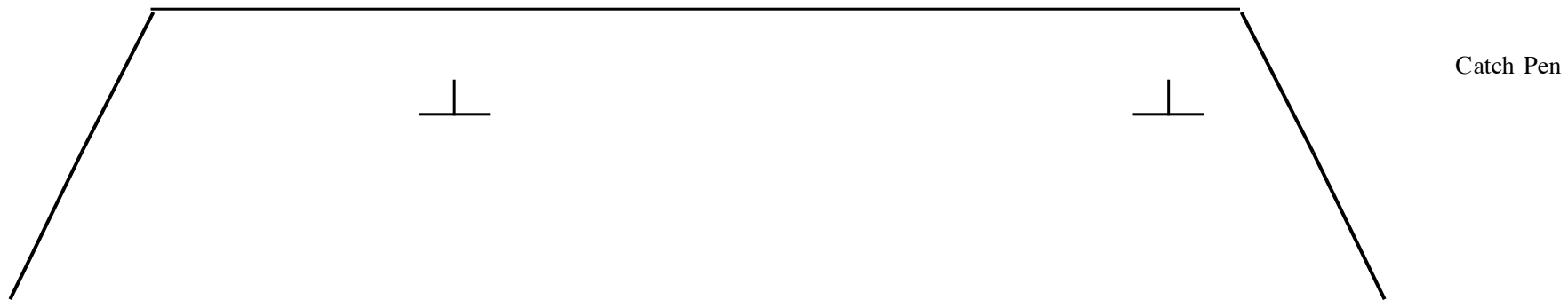

Fig. 2.1: Behavior test setup. The chute measured 1.5 meters wide and 7 meters long; not to scale. 


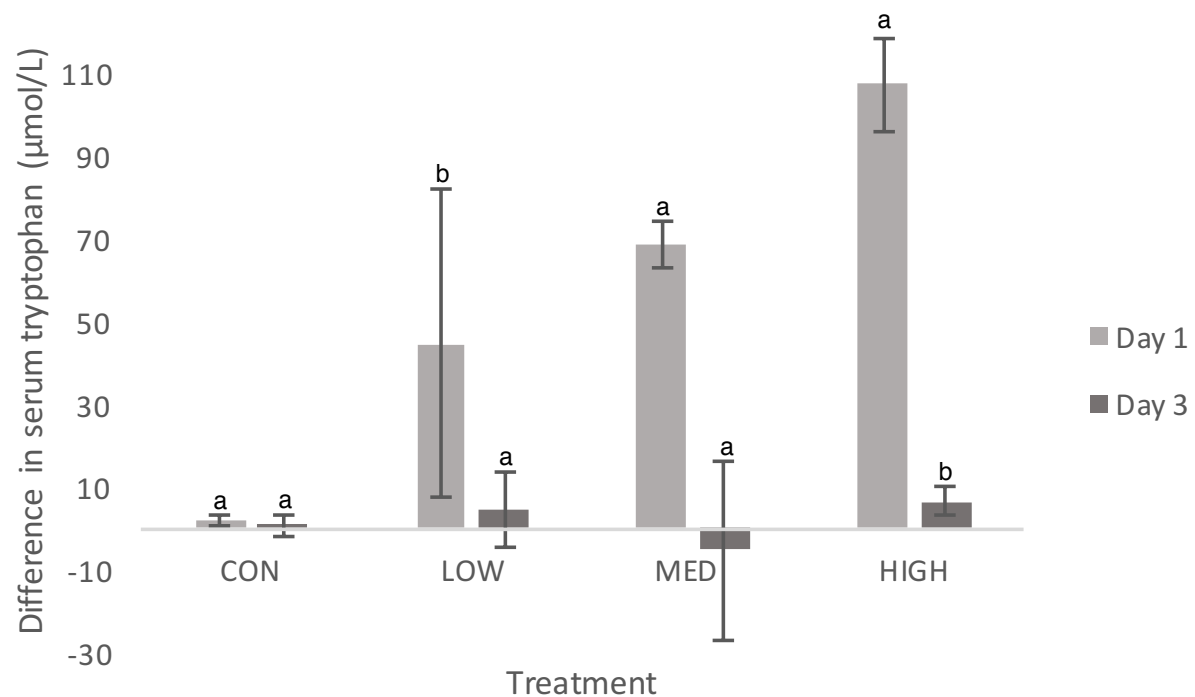

Fig. 2.2: Average ( \pm s.d.) difference in serum free tryptophan ( $\mu$ mol/L) for Group 1 horses calculated by subtracting levels in BC1 sample from levels BC2 sample. ${ }^{a} n=4 .{ }^{b} n=3$.

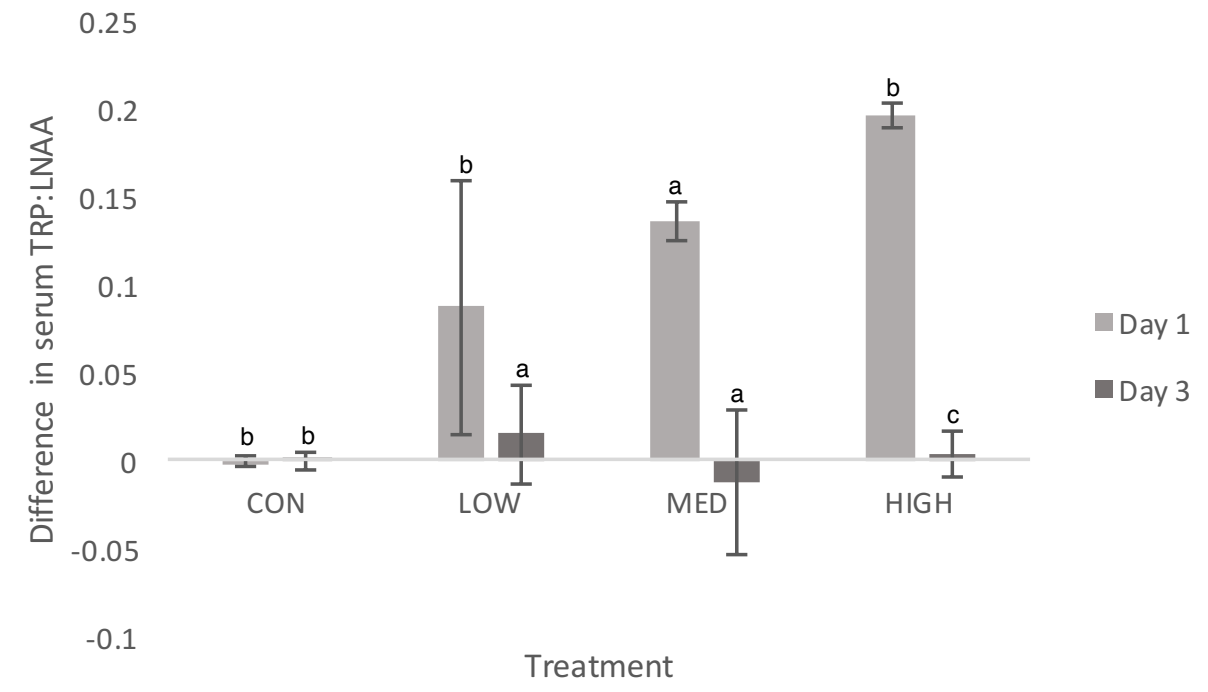

Fig. 2.3: Average ( \pm s.d.) difference in serum Trp:LNAA for Group 1 horses calculated by subtracting ratio in BC1 samples from ratio in $B C 2$ sample. ${ }^{a} n=4 .{ }^{b} n=3 .{ }^{c} n=2$. 


\section{LITERATURE CITED}

Adeola, O. \& Ball, R. O. 1992. Hypothalamic neurotransmitter concentrations and meat quality in stressed pigs offered excess dietary tryptophan and tyrosine. Journal of Animal Science, 70, 1888-1894.

Alberghina, D., Giannetto, C. \& Piccione, G. 2010a. Peripheral serotoninergic response to physical exercise in athletic horses. Journal of Veterinary Science, 11, 285-289.

Alberghina, D., Giannetto, C., Visser, E. K. \& Ellis, A. D. 2010b. Effect of diet on plasma tryptophan and serotonin in trained mares and geldings. Vet Rec, 166, 133-6.

Alberghina, D., Panzera, M., Giannetto, C. \& Piccione, G. 2014. Developmental changes during the first year of life in plasma tryptophan concentration of the foal. Journal of Equine Veterinary Science, 34, 387-390.

Albonetti, M. E., Gonzalez, M. I., Wilson, C. A. \& Farabollini, F. 1994. Effects of neonatal treatment with 1-(2, 5-dimethoxy-4-lodophenyl)-2 aminopropane HCI (DOI) and ritanserin on agonistic behavior in adult male and female rats. Aggressive Behavior, 20, $135-242$.

Alenka, N.-S., Nina, C.-K. \& P, K. 2008. Influence of a calmative on selected blood parameters in horses under stressful conditions. Acta veterinaria, 58, 509-519.

Argyropoulos, S. V., Hood, S. D., Adrover, M., Bell, C. J., Rich, A. S., Nash, J. R., Rich, N. C., Witchel, H. J. \& Nutt, D. J. 2004. Tryptophan depletion reverses the therapeutic effect of selective serotonin reuptake inhibitors in social anxiety disorder. Biol Psychiatry, 56, 503-9.

Assenza, A., Bergero, D., Tarantola, M., Piccione, G. \& Caola, G. 2004. Blood serum branched chain amino acids and tryptophan modifications in horses competing in long-distance rides of different length. Journal of Animal Physiology and Animal Nutrition, 88, 172177.

Bagshaw, C. S., Ralston, S. L. \& Fisher, H. 1994. Behavioral and physiological effect of orally administered tryptophan on horses subjected to acute isolation stress. Applied Animal Behaviour Science, 40, 1-12.

Basic, D., Krogdahl, Å., Schjolden, J., Winberg, S., Vindas, M. A., Hillestad, M., Mayer, I., Skjerve, E. \& Höglund, E. 2013. Short- and long-term effects of dietary l-tryptophan supplementation on the neuroendocrine stress response in seawater-reared Atlantic salmon (Salmo salar). Aquaculture, 388-391, 8-13.

Bell, C., Abrams, J. \& Nutt, D. 2001. Tryptophan depletion and its implications for psychiatry. The British Journal of Psychiatry, 178, 399-405.

Bellisle, F., Blundell, J. E., Dye, L., Fantino, M., Fern, E., Fletcher, R. J., Lambert, J., Roberfroid, M., Specter, S., Westenhofer, J. \& Westerterp-Plantenga, M. S. 1998. 
Functional food science and behaviour and psychological functions. British Journal of Nutrition, 80, S173-S193.

Bergero, D., Assenza, A., Schiavone, A., Piccione, G., Perona, G. \& Caola, G. 2005. Amino acid concentrations in blood serum of horses performing long lasting low-intensity exercise. Journal of Animal Physiology and Animal Nutrition, 89, 146-150.

Boadle-Biber, M. 1993. Regulation of serotonin synthesis. Progress in Biophysics and Molecular Biology, 60, 1-15.

Bosch, G., Beerda, B., Hendriks, W. H., van der Poel, A. F. \& Verstegen, M. W. 2007. Impact of nutrition on canine behaviour: current status and possible mechanisms. Nutr Res Rev, 20, $180-94$.

Boyer, E. W. \& Shannon, M. 2005. The serotonin syndrome. The New England Journal of Medicine, 352, 1112-1120.

Breedlove, S. M., Watson, N. V. \& Rosenzweig, M. R. 2010. Biological Psychology: An Introduction to Behavioral, Cognitive, and Clinical Neuroscience, 6th edn. Sinauer Associates, Sunderland, MA.

Bruschetta, G., Di Pietro, P., Miano, M., Zanghi, G., Fazio, E. \& Ferlazzo, A. M. 2013. Daily variations of plasma serotonin levels in 2-year-old horses. Journal of Veterinary Behavior: Clinical Applications and Research, 8, 95-99.

Chamberlain, B., Ervin, F. R., Pihl, R. O. \& Young, S. N. 1987. The effect of raising or lowering tryptophan levels on aggression in vervet monkeys. Pharmacology Biochemistry and Behavior, 28, 503-510.

Corr, P. J. 2006. Understanding Biological Psychology. Blackwell Publishing, Malden, MA.

Davis, J. M., Alderson, N. L. \& Welsh, R. S. 2000. Serotonin and central nervous system fatigue: nutritional considerations. American Journal of Clinical Nutrition, 72, 573s-578s.

DeNapoli, J. S., Dodman, N. H., Shuster, L., Rand, W. M. \& Gross, K. L. 2000. Effect of dietary protein content and tryptophan supplementation on dominance aggression, territorial aggression, and hyperactivity in dogs. Journal of American Veterinary Association, 217, $504-508$.

Dickinson, S. L. \& Curzon, G. 1986. 5-hydroxytryptamine-mediated behaviour in male and female rats. Neuropharmacology, 25, 771-776.

Esteban, S., Nicolaus, C., Garmundi, A., Rial, R. V., Rodriguez, A. B., Ortega, E. \& Ibars, C. B. 2004. Effect of orally administered L-tryptophan on serotonin, melatonin, and the innate immune response in the rat. Molecular and Cellular Biochemistry, 267, 39-46. 
Farabollini, F., Hole, D. R. \& Wilson, C. A. 1988. Behavioral Effects in Adulthood of Serotonin Depletion by P-Chlorophenylalanine Given Neonatally to Male Rats. International Journal of Neuroscience, 41, 187-199.

Farris, J. W., Hinchcliff, K. W., McKeever, K. H., Lamb, D. R. \& Thompson, D. L. 1998. Effect of tryptophan and of glucose on exercise capacity of horses. Journal of Applied Physiology, 85, 807-816.

Ferlazzo, A. M., Bruschetta, G., Di Pietro, P.\& Medica, P. 2012. The influence of age on plasma serotonin levels in Thoroughbred horses. Livestock Science, 147, 203-207.

Fernstrom, J. D. 2013. Large neutral amino acids: dietary effects on brain neurochemistry and function. Amino Acids, 45, 419-30.

Fernstrom, J. D. \& Wurtman, R. J. 1972a. Brain Serotonin Content: Physiological Regulation by Plasma Neutral Amino Acids. Science, 178, 414-416.

Fernstrom, J. D. \& Wurtman, R. J. 1972b. Elevation of plasma tryptophan by insulin in rat. Metabolism, 21, 337-342.

Fernstrom, J. D. \& Wurtman, R. J. 1974. Control of brain serotonin levels by the diet. Advances in Biochemical Psychopharmacology, 11, 133-142.

Gillman, P. K. 1999. The serotonin syndrome and its treatment. Journal of Psychopharmacology, 13, 100-109.

Goldstein, G. W. 1988. Endothelial cell $\square$-astrocyte interactions: a cellular model of the bloodbrain barrier. Annals of the New York Academy of Sciences, 529, 31-39.

Grimmett, A. \& Sillence, M. N. 2005. Calmatives for the excitable horse: a review of Ltryptophan. Vet $J, 170,24-32$.

Guyton, A. C. \& Hall, J. E. 2000. Textbook of Medical Physiology, 10th edn. Saunders Company, Philadelphia, PA.

Hannon, J. \& Hoyer, D. 2008. Molecular biology of 5-HT receptors. Behav Brain Res, 195, 198213.

Henneke, D. R., Potter, G. D., Kreider, J. L. \& Yeates, B. F. 1983. Relationship between condition score, physical measurements and body fat percentage in mares. Equine Veterinary Journal, 15, 371-372.

Henry, Y., Seve, B., Colléaux, Y., Ganier, P., Saligaut, C. \& Jégo, P. 1992. Interactive effects of dietary levels of tryptophan and protein on voluntary feed intake and growth in pigs, in relation to plasma free amino acids and hypothalamic serotonin. Journal of Animal Science, 70, 1873-1887. 
Henry, Y., Seve, B., Mounier, A. \& Ganier, P. 1996. Growth performance and brain neurotransmitters in pigs as affected by tryptophan, protein, and sex. Journal of Animal Science, 74, 2700-2710.

Hilakivi-Clarke, L. A., Durcan, M. J., Lister, R. G. \& Linnoila, M. 1990. Effect of tryptophan on the behavior of nonstressed and stressed mice in Porsolt's swim test. Pharmacology Biochemistry and Behavior, 37, 273-276.

Janczak, A. M., Bakken, M. \& Braastad, B. O. 2001. A cautionary note regarding the use of nutritional L-tryptophan to alter aversion-related behaviour in mice. Applied Animal Behaviour Science, 72, 365-373.

Kahn, C. M. \& Line, S. (eds.) 2010. The Merck Veterinary Manual, Whitehouse Station, N.J., Merck \& CO., Inc.

Kantak, K. M., Hegstrand, L. R., Whitman, J. \& Eichelman, B. 1980. Effects of dietary supplements and a tryptophan-free diet on aggressive behavior in rats. Pharmacology Biochemistry and Behavior, 12, 173-179.

Kennett, G. A., Chaouloff, F., Marcou, M. \& Curzon, G. 1986. Female rats are more vulnerable than males in an animal model of depression: the possible role of serotonin. Brain Research, 382, 416-421.

Kim, D. Y. \& Camilleri, M. 2000. Serotonin: A Mediator of the Brain-Gut Connection. The American Journal of Gastroenterology, 95, 2698-2709.

Koopmans, S. J., Ruis, M., Dekker, R., van Diepen, H., Korte, M. \& Mroz, Z. 2005. Surplus dietary tryptophan reduces plasma cortisol and noradrenaline concentrations and enhances recovery after social stress in pigs. Physiol Behav, 85, 469-78.

Laycock, S. R. \& Ball, R. O. 1990. Alleviation of hysteria in laying hens with dietary tryptophan. Canadian Journal of Veterinary Research, 54, 291-295.

Leathwood, P. D. 1987. Tryptophan availability and serotonin synthesis. Proceedings of the Nutrition Society, 46, 143-156.

Li, Y. Z., Kerr, B. J., Kidd, M. T. \& Gonyou, H. W. 2006. Use of supplementary tryptophan to modify the behavior of pigs. Journal of Animal Science, 84, 212-220.

Lieberman, H. R., Caballero, B. \& Finer, N. 1986. The composition of lunch determines afternoon plasma tryptophan ratios in humans. Journal of neural transmission, 65, 211217.

Malmkvist, J. \& Christensen, J. W. 2007. A note on the effects of a commercial tryptophan product on horse reactivity. Applied Animal Behaviour Science, 107, 361-366.

Martinez-Trejo, G., Ortega-Cerrilla, M. E., Rodarte-Covarrubias, L. F., Herrera-Haro, J. G., Figueroa-Velasco, J. L., Galindo-Maldonado, F., Sanchez-Martinez, O. \& Lara-Bueno, 
A. 2009. Aggressiveness and productive performance of piglets supplemented with tryptophan. Journal of Animal and Verterinary Advances, 8, 608-611.

Mench, J. A. \& Shea-Moore, M. M. 1995. Moods, minds and molecules: the neurochemistry of social behavior. Applied Animal Behaviour Science, 44, 999-118.

Momozawa, Y., Takeuchi, Y., Tozaki, T., Kikusui, T., Hasegawa, T., Raudsepp, T., Chowdhary, B. P., Kusunose, R. \& Mori, Y. 2006. Polymorphism identification, RH mapping, and association analysis with the anxiety trait of the equine serotonin transporter (SLC6A4) gene. Journal of Veterinary Medical Science, 68, 619-621.

Nakanishi, Y., Shigemori, K., Yanagita, K., Mieno, M. \& Manda, M. 1998. Behavioral and growth effect of oral administration of rumen protected tryptophan on weanling beef calves. Memoirs of the Faculty of Agriculture, Kagoshima University, 34, 89-95.

Noble, G. K., Blackshaw, K. L., Cowling, A., Harris, P. A. \& Sillence, M. N. 2013. An objective measure of reactive behaviour in horses. Applied Animal Behaviour Science, 144, 121129.

Noble, G. K., Brockwell, Y. M., Munn, K. J., Harris, P. A., Davidson, H. P., Li, X., Zhang, D. \& Sillence, M. N. 2008. Effects of a commercial dose of L-tryptophan on plasma tryptophan concentrations and behaviour in horses. Equine Vet J, 40, 51-6.

Noble, G. K., Houghton, E., Roberts, C. J., Faustino-Kemp, J., de Kock, S. S., Swanepoel, B. C. \& Sillence, M. N. 2007. Effect of exercise, training, circadian rhythm, age, and sex on insulin-like growth factor-1 in the horse. J Anim Sci, 85, 163-71.

Nogueira, N. P., Barnabe, R. C., Bedran-De-Castro, J. C., Moreira, A. F., Fernandes, W. R., Mirandola, R. M. S. \& Howard, D. L. 2002. Serum cortisol, lactate, and creatinine concentrations in Thoroughbred fillies of different ages and states of training. Braz. $J$. vet. Res.anim.Sci., 39, 54-57.

NRC. 2007. Nutrient Requirements of Horses, 6th rev. edn. The National Academies Press, Washington, D.C.

O'Reilly, B. 2006. Reducing cribbing frequency in horses through dietary supplementation of tryptophan and calcium carbonate. Animal Science. Auburn University.

Paradis, M. R., Breeze, R. G., Bayly, W. M., Counts, D. F. \& Laegreid, W. W. 1991. Acute hemolytic anemia after oral administration of L-tryptophan in ponies. American Journal of Veterinary Research, 52, 742-747.

Peeters, E., Driessen, B., Steegmans, R., Henot, D. \& Geers, R. 2004. Effect of supplemental tryptophan, vitamin E, and a herbal product on responses by pigs to vibration. Journal of Animal Science, 82, 2410-2420. 
Piccione, G., Assenza, A., Fazio, F., Percipalle, M. \& Caola, G. 2005. Central fatigue and nycthemeral change of serum tryptophan and serotonin in the athletic horse. J Circadian Rhythms, 3, 6 .

Poletto, R., Kretzer, F. C. \& Hötzel, M. J. 2014. Minimizing aggression during mixing of gestating sows with supplementation of a tryptophan-enriched diet. Physiol Behav, 132, $36-43$.

Raleigh, M. J. 1987. Differential behavioral effects of tryptophan and 5-hydroxytryptophan in vervet monkeys: influence of catecholaminergic systems. Psychopharmacology, 93, 4450 .

Rapoport, S. I. 1976. Blood-brain barrier in physiology and medicine. Raven Press, New York.

Robbins, T. W. 2005. Controlling stress: how the brain protects itself from depression. Nature Neuroscience, 8, 261-262.

Rouvinen, K., Archbold, S., Laffin, S. \& Harri, M. 1999. Long-term effects of tryptophan on behavioral response and growing-furring performance in silver fox (Vulpes vulpes). Applied Animal Behaviour Science, 63, 65-77.

Schaechter, J. D. \& Wurtman, R. J. 1990. Serotonin release varies with brain tryptophan levels. Brain Research, 532, 203-210.

Shea, M. M., Mench, J. A. \& Thomas, O. P. 1990. The effect of dietary tryptophan on aggressive behavior in developing and mature broiler breeder males. Poultry Science, 69, 16641669.

Shen, Y. B., Coffey, M. T.\& Kim, S. W. 2015. Effects of short term supplementation of 1tryptophan and reducing large neutral amino acid along with l-tryptophan supplementation on growth and stress response in pigs. Animal Feed Science and Technology, 207, 245-252.

Speake, T., Whitwell, C., Kajita, H., Majid, A. \& Brown, P. D. 2000. Mechanisms of CSF secretion by the choroid plexus. Microscopy Research and Technique, 52, 49-59.

Sternbach, H. 1991. The serotonin syndrome. American Journal of Psychiatry, 148, 705-713.

Stull, C. L. \& Rodiek, A. V. 2000. Physiological responses of horses to 24 hours of transportation using a commercial van during summer conditions. Journal of Animal Science, 78, 1458-1466.

Trulson, M. E. \& Jacobs, B. L. 1979. Raphe unit activity in freely moving cats: correlation with level of behavioral arousal. Brain Research, 163, 135-150.

USEF. 2015. 2015 Guidelines for Drugs and Medications. In: Program, U. S. E. F. E. D. a. M. (ed.). Columbus, Ohio. 
Vervuert, I., Coenen, M. \& Watermulder, E. 2005. Metabolic responses to oral tryptophan supplementation before exercise in horses. Journal of Animal Physiology and Animal Nutrition, 89, 140-145.

Voet, D. \& Voet, J. G. 1995. Biochemistry, 2nd edn. John Wiley \& Sons, Inc., New York.

Weed, L. W. 1923. The absorption of cerebrospinal fluid into the venous system. American Journal of Anatomy, 31, 191-221.

Weld, K. P., Mench, J. A., Woodward, R. A., Bolesta, M. S., Suomi, S. J. \& Higley, J. D. 1998. Effect of tryptophan treatment on self-biting and central nervous system serotonin metabolism in rhesus monkeys (Macaca mulatta). Neuropsychopharmacology, 19, 314321.

Wilson, A. D., Badnell-Waters, A. J., Bice, R., Kelland, A., Harris, P. A. \& Nicol, C. J. 2007. The effects of diet on blood glucose, insulin, gastrin and the serum tryptophan: large neutral amino acid ratio in foals. Vet $J, 174,139-46$.

Wurtman, R. J. 2011. Non-nutritional uses of nutrients. Eur J Pharmacol, 668 Suppl 1, S10-5.

Yao, K., Fang, J., Yin, Y. L., Feng, Z. M., Tang, Z. R. \& Wu, G. 2011. Tryptophan metabolism in animals: important roles in nutrition and health. Frontiers in Bioscience, 3, 286-297.

Young, S. N. 1986. The clinical pyschopharmacology of tryptophan. Nutrition and the brain (USA).

Young, S. N. 1991. Some effects of dietary components (amino acids, carbohydrate, folic acid) on brain serotonin synthesis, mood, and behavior. Canadian Journal of Physiology and Pharmacology, 69, 893-903.

Zhang, D., Li, X., Sillence, M. N. \& Bryden, W. L. 2005. Serum amino acid analysis by gas chromatography. Asia Pacific J. Clin. Nutr. Suppl., 14, S121. 


\section{APPENDIX 1}

\section{SAS CODE}

Analyze Treatment Effects on Behavioral and Physiological Response Variables on Day 1

PROC IMPORT OUT=WORK.AllDay1Data DATAFILE="/home/britdav/AllDay1Data.xlsx" DBMS=XLSX REPLACE;

GETNAMES=YES;

run;

proc sort data=AllDay1Data;

by Horse;

run;

*Summary Plots;

Proc Means data=AllDay1Data nway noprint;

class Treatment Day; var ExitSpeed HR30 HR_Diff TimetoBL GluDiff GluPostAvg LactDiff LactPostAvg CortDiff CortPostAvg;

output out=SumStats mean=;

run;

Proc Transpose data $=$ SumStats out $=\operatorname{SumStats} \operatorname{Tr}($ rename $=(\mathrm{Col1}=\mathrm{Y}))$;

by Treatment Day_TYPE__FREQ_;

run;

Proc sort data=SumStatsTr;

by _LABEL_;

*Simple interaction plot for each response variable;

Proc Sgplot ;

by _LABEL_;

series $\mathrm{X}=$ Treatment $\mathrm{Y}=\mathrm{Y}$;

run;

*proc corr data=AllDay1Data plots=scatter;

* $\quad$ var treatment;

* with exitspeed;

*run;

*proc corr data=AllDay1Data plots=scatter;

* var treatment;

* $\quad$ with HR30; 
*run;

*proc corr data=AllDay1Data plots=scatter;

* $\quad$ var treatment;

* with HR_Diff;

*run;

*proc corr data=AllDay1Data plots=scatter;

* $\quad$ var treatment;

* $\quad$ with TimetoBL;

*run;

*proc corr data=AllDay1Data plots=scatter;

* var treatment;

* with GluDiff;

*run;

*proc corr data=AllDay1Data plots=scatter;

* var treatment;

* with GluPostAvg;

*run;

*proc corr data=AllDay1Data plots=scatter;

* $\quad$ var treatment;

* $\quad$ with LactDiff;

*run;

*proc corr data=AllDay1Data plots=scatter;

* $\quad$ var treatment;

* with LactPostAvg;

*run;

*proc corr data=AllDay1Data plots=scatter;

* var treatment;

* $\quad$ with CortDiff;

*run;

*proc corr data=AllDay1Data plots=scatter;

* $\quad$ var treatment;

* with CortPostAvg;

*run;

*Covariate tests;

proc corr data=AllDay1Data plots=scatter; var Age; 
with ExitSpeed HR30 HR_Diff TimetoBL GluDiff GluPostAvg LactDiff LactPostAvg CortDiff CortPostAvg;

run;

proc corr data=AllDay 1 Data plots $=$ scatter;

var BCS;

with ExitSpeed HR30 HR_Diff TimetoBL GluDiff GluPostAvg LactDiff LactPostAvg CortDiff CortPostAvg;

run;

proc corr data=AllDay 1 Data plots $=$ scatter;

var Group;

with ExitSpeed HR30 HR_Diff TimetoBL GluDiff GluPostAvg LactDiff LactPostAvg CortDiff CortPostAvg;

run;

proc corr data=AllDay 1 Data plots=scatter;

var TimeLag;

with ExitSpeed HR30 HR_Diff TimetoBL GluDiff GluPostAvg LactDiff LactPostAvg CortDiff CortPostAvg;

run;

proc corr data=AllDay 1 Data plots=scatter;

var TrtNum;

with ExitSpeed HR30 HR_Diff TimetoBL GluDiff GluPostAvg LactDiff LactPostAvg CortDiff CortPostAvg;

run;

proc corr data=AllDay 1 Data plots=scatter;

var Temperature;

with ExitSpeed HR30 HR_Diff TimetoBL GluDiff GluPostAvg LactDiff LactPostAvg CortDiff CortPostAvg;

run;

proc corr data=AllDay 1 Data plots=scatter;

var WindSpeed;

with ExitSpeed HR30 HR_Diff TimetoBL GluDiff GluPostAvg LactDiff LactPostAvg CortDiff CortPostAvg;

run;

proc corr data=AllDay 1 Data plots=scatter;

var Breed;

with ExitSpeed HR30 HR_Diff TimetoBL GluDiff GluPostAvg LactDiff LactPostAvg CortDiff CortPostAvg;

run; 
proc corr data=AllDay1Data plots=scatter; var GluDiff GluPostAvg; with GluRunDate;

run;

proc corr data=AllDay1Data plots=scatter; var LactDiff LactPostAvg; with LactRunDate;

run;

proc corr data=AllDay1Data plots=scatter; var CortDiff CortPostAvg; with CortPretestPlate CortPosttestPlate;

run;

*Overview Stats;

proc means data=AllDay1Data mean std; class treatment; var exitspeed HR30 HR_Diff TimetoBL GluDiff GluPostAvg LactDiff LactPostAvg CortDiff CortPostAvg;

run;

proc univariate data=AllDay1Data plots;

var ExitSpeed HR30 HR_Diff TimetoBL GluDiff GluPostAvg LactDiff LactPostAvg CortDiff CortPostAvg;

run;

title 'Day 1 Exit Speed';

Proc Mixed data=AllDay1Data covtest ratio;

class Treatment Group Horse;

model ExitSpeed=Group Temperature Treatment/ ddfm=kr solution residual;

random Horse;

lsmeans Treatment / pdiff;

lsmestimate Treatment $3-1-1-1 /$ divisor $=3$;

run;

title 'Day 1 HR 30';

Proc Mixed data=AllDay1Data covtest ratio;

class Treatment TrtNum Horse;

model HR30=TrtNum Treatment/ ddfm=kr solution residual;

random Horse;

lsmeans Treatment / pdiff;

lsmestimate Treatment $3-1-1-1 /$ divisor $=3$;

run; 
title 'Day 1 HR Difference';

Proc Mixed data=AllDay 1Data covtest ratio;

class Treatment Group TrtNum Horse;

model HR_Diff=Group TrtNum Treatment/ ddfm=kr solution residual;

random Horse;

lsmeans Treatment / pdiff;

lsmestimate Treatment $3-1-1-1 /$ divisor $=3$;

run;

title 'Day 1 Time to Return to Baseline';

Proc Mixed data=AllDay1Data covtest ratio;

class Treatment Horse;

model TimetoBL $=$ Temperature Treatment $/ \mathrm{ddfm}=\mathrm{kr}$ solution residual;

random Horse;

lsmeans Treatment / pdiff;

lsmestimate Treatment $3-1-1-1 /$ divisor $=3$;

run;

title 'Day 1 Glucose Difference';

Proc Mixed data=AllDay1Data covtest ratio;

class Treatment GluRunDate Horse;

model GluDiff=GluRunDate TimeLag Treatment/ $\mathrm{ddfm}=\mathrm{kr}$ solution residual;

random Horse;

lsmeans Treatment / pdiff;

lsmestimate Treatment $3-1-1-1 /$ divisor $=3$;

run;

title 'Day 1 Glucose Post-Startle Average';

Proc Mixed data=AllDay1Data covtest ratio;

class Treatment Horse;

model GluPostAvg= TimeLag Treatment/ ddfm=kr solution residual;

random Horse;

lsmeans Treatment / pdiff;

lsmestimate Treatment $3-1-1-1 /$ divisor $=3$;

run;

title 'Day 1 Lactate Difference';

Proc Mixed data=AllDay1Data covtest ratio;

class Treatment Horse;

model LactDiff=Treatment $/ \mathrm{ddfm}=\mathrm{kr}$ solution residual;

random Horse;

lsmeans Treatment / pdiff;

lsmestimate Treatment 3-1 -1 -1/ divisor $=3$;

run;

title 'Day 1 Lactate Post-Startle Average'; 
Proc Mixed data=AllDay1Data covtest ratio;

class Treatment Horse;

model LactPostAvg=Treatment/ ddfm=kr solution residual;

random Horse;

lsmeans Treatment / pdiff;

lsmestimate Treatment $3-1-1-1 /$ divisor $=3$;

run;

title 'Day 1 Cortisol Difference';

Proc Mixed data=AllDay1Data covtest ratio;

class Treatment Horse;

model CortDiff=Treatment/ ddfm=kr solution residual;

random Horse;

lsmeans Treatment / pdiff;

lsmestimate Treatment $3-1-1-1 /$ divisor $=3$;

run;

title 'Day 1 Cortisol Post-Startle Average';

Proc Mixed data=AllDay1Data covtest ratio;

class Treatment Horse CortPostTestPlate;

model CortPostAvg=CortPosttestPlate Treatment/ ddfm=kr solution residual;

random Horse;

lsmeans Treatment / pdiff;

lsmestimate Treatment $3-1-1-1 /$ divisor $=3$;

run; 


\section{APPENDIX 2}

\section{SAS CODE}

Analyze Treatment Effects on Behavioral and Physiological Response Variables on Day 3

PROC IMPORT OUT=WORK.AllDay3Data DATAFILE="/home/britdav/AllDay3Data.xlsx"

DBMS=XLSX REPLACE; GETNAMES=YES;

run;

proc sort data=AllDay3Data;

by Horse;

run;

*Summary Plots;

Proc Means data=AllDay3Data nway noprint;

class Treatment Day; var ExitSpeed HR30 HR_Diff TimetoBL GluDiff GluPostAvg LactDiff LactPostAvg CortDiff CortPostAvg;

output out=SumStats mean=;

run;

Proc Transpose data $=$ SumStats out $=$ SumStatsTr $($ rename $=(\operatorname{Col} 1=Y))$;

by Treatment Day_TYPE__FREQ_;

run;

Proc sort data=SumStatsTr;

by _LABEL_;

*Simple interaction plot for each response variable;

Proc Sgplot ;

by _LABEL_;

series $\mathrm{X}=$ Treatment $\mathrm{Y}=\mathrm{Y}$;

run;

*proc corr data=AllDay3Data plots=scatter;

* $\quad$ var treatment;

* $\quad$ with exitspeed;

*run;

*proc corr data=AllDay3Data plots=scatter;

* var treatment;

* with HR30; 
*run;

*proc corr data=AllDay3Data plots=scatter;

* $\quad$ var treatment;

* with HR_Diff;

*run;

*proc corr data=AllDay3Data plots=scatter;

* $\quad$ var treatment;

* $\quad$ with TimetoBL;

*run;

*proc corr data=AllDay3Data plots=scatter;

* var treatment;

* with GluDiff;

*run;

*proc corr data=AllDay3Data plots=scatter;

* var treatment;

* with GluPostAvg;

*run;

*proc corr data=AllDay3Data plots=scatter;

* $\quad$ var treatment;

* $\quad$ with LactDiff;

*run;

*proc corr data=AllDay3Data plots=scatter;

* $\quad$ var treatment;

* with LactPostAvg;

*run;

*proc corr data=AllDay3Data plots=scatter;

* var treatment;

* $\quad$ with CortDiff;

*run;

*proc corr data=AllDay3Data plots=scatter;

* $\quad$ var treatment;

* with CortPostAvg;

*run;

*Covariate tests;

proc corr data=AllDay3Data plots=scatter; var Age; 
with ExitSpeed HR30 HR_Diff TimetoBL GluDiff GluPostAvg LactDiff LactPostAvg CortDiff CortPostAvg;

run;

proc corr data=AllDay3Data plots=scatter;

var BCS;

with ExitSpeed HR30 HR_Diff TimetoBL GluDiff GluPostAvg LactDiff LactPostAvg CortDiff CortPostAvg;

run;

proc corr data=AllDay3Data plots=scatter;

var Group;

with ExitSpeed HR30 HR_Diff TimetoBL GluDiff GluPostAvg LactDiff LactPostAvg CortDiff CortPostAvg;

run;

proc corr data=AllDay3Data plots=scatter;

var TimeLag;

with ExitSpeed HR30 HR_Diff TimetoBL GluDiff GluPostAvg LactDiff LactPostAvg CortDiff CortPostAvg TrtNum;

run;

proc corr data=AllDay3Data plots=scatter;

var TrtNum;

with ExitSpeed HR30 HR_Diff TimetoBL GluDiff GluPostAvg LactDiff LactPostAvg CortDiff CortPostAvg TimeLag;

run;

proc corr data=AllDay3Data plots=scatter;

var Temperature;

with ExitSpeed HR30 HR_Diff TimetoBL GluDiff GluPostAvg LactDiff LactPostAvg CortDiff CortPostAvg;

run;

proc corr data=AllDay3Data plots=scatter;

var WindSpeed;

with ExitSpeed HR30 HR_Diff TimetoBL GluDiff GluPostAvg LactDiff LactPostAvg CortDiff CortPostAvg;

run;

proc corr data=AllDay3Data plots=scatter;

var Breed;

with ExitSpeed HR30 HR_Diff TimetoBL GluDiff GluPostAvg LactDiff LactPostAvg CortDiff CortPostAvg;

run; 
proc corr data=AllDay3Data plots=scatter; var GluDiff GluPostAvg; with GluRunDate;

run;

proc corr data=AllDay3Data plots=scatter; var LactDiff LactPostAvg; with LactRunDate;

run;

proc corr data=AllDay3Data plots $=$ scatter; var CortDiff CortPostAvg;

with CortPretestPlate CortPosttestPlate;

run;

*Overview Stats;

proc means data=AllDay3Data mean std;

class treatment; var exitspeed HR30 HR_Diff TimetoBL GluDiff GluPostAvg LactDiff LactPostAvg CortDiff CortPostAvg;

run;

proc univariate data=AllDay3Data plots;

var ExitSpeed HR30 HR_Diff TimetoBL GluDiff GluPostAvg LactDiff LactPostAvg CortDiff CortPostAvg;

run;

title 'Day 3 Exit Speed';

Proc Mixed data=AllDay3Data covtest ratio;

class Treatment Group Horse;

model ExitSpeed=Group Temperature Treatment $/ \mathrm{ddfm}=\mathrm{kr}$ solution residual;

random Horse;

lsmeans Treatment / pdiff;

lsmestimate Treatment $3-1-1-1 /$ divisor $=3$;

run;

title 'Day 3 HR 30';

Proc Mixed data=AllDay3Data covtest ratio; class Treatment TrtNum Horse;

model HR30 = TrtNum Treatment/ ddfm=kr solution residual;

random Horse;

lsmeans Treatment / pdiff;

lsmestimate Treatment $3-1-1-1 /$ divisor $=3$;

run; 
title 'Day 3 HR Difference';

Proc Mixed data=AllDay3Data covtest ratio;

class Treatment Group TrtNum Horse;

model HR_Diff=Group TrtNum Treatment/ ddfm=kr solution residual;

random Horse;

lsmeans Treatment / pdiff;

lsmestimate Treatment $3-1-1-1 /$ divisor $=3$;

run;

title 'Day 3 Time to Return to Baseline';

Proc Mixed data=AllDay3Data covtest ratio;

class Treatment Horse;

model TimetoBL $=$ Temperature Treatment $/ \mathrm{ddfm}=\mathrm{kr}$ solution residual;

random Horse;

lsmeans Treatment / pdiff;

lsmestimate Treatment $3-1-1-1 /$ divisor $=3$;

run;

title 'Day 3 Glucose Difference';

Proc Mixed data=AllDay3Data covtest ratio;

class Treatment GluRunDate Horse;

model GluDiff=GluRunDate TimeLag Treatment/ $\mathrm{ddfm}=\mathrm{kr}$ solution residual;

random Horse;

lsmeans Treatment / pdiff;

lsmestimate Treatment $3-1-1-1 /$ divisor $=3$;

run;

title 'Day 3 Glucose Post-Startle Average';

Proc Mixed data=AllDay3Data covtest ratio;

class Treatment Horse;

model GluPostAvg=TimeLag Treatment/ ddfm=kr solution residual;

random Horse;

lsmeans Treatment / pdiff;

lsmestimate Treatment $3-1-1-1 /$ divisor $=3$;

run;

title 'Day 3 Lactate Difference';

Proc Mixed data=AllDay3Data covtest ratio;

class Treatment Horse;

model LactDiff=Treatment $/ \mathrm{ddfm}=\mathrm{kr}$ solution residual;

random Horse;

lsmeans Treatment / pdiff;

lsmestimate Treatment $3-1-1-1 /$ divisor $=3$;

run;

title 'Day 3 Lactate Post-Startle Average'; 
Proc Mixed data=AllDay3Data covtest ratio;

class Treatment Horse;

model LactPostAvg=Treatment/ ddfm=kr solution residual;

random Horse;

lsmeans Treatment / pdiff;

lsmestimate Treatment $3-1-1-1 /$ divisor $=3$;

run;

title 'Day 3 Cortisol Difference';

Proc Mixed data=AllDay3Data covtest ratio;

class Treatment Horse;

model CortDiff=Treatment/ ddfm=kr solution residual;

random Horse;

lsmeans Treatment / pdiff;

lsmestimate Treatment $3-1-1-1 /$ divisor $=3$;

run;

title 'Day 3 Cortisol Post-Startle Average';

Proc Mixed data=AllDay3Data covtest ratio;

class Treatment Horse CortPosttestPlate;

model CortPostAvg=CortPosttestPlate Treatment/ ddfm=kr solution residual;

random Horse;

lsmeans Treatment / pdiff;

lsmestimate Treatment $3-1-1-1 /$ divisor $=3$;

run; 


\section{APPENDIX 3}

\section{SAS CODE}

Analyze Serum Amino Acids of Group 1 Horses

PROC IMPORT OUT=WORK.Group1 DATAFILE="/home/britdav/Group1.xlsx"

DBMS=XLSX REPLACE;

GETNAMES=YES;

RUN;

*Summary Plots;

Proc Means data $=$ Group1 nway;

class Treatment Day;

var TRP_Diff RatioDiff;

output out=SumStatsGroup1 mean= ;

run;

Proc Transpose data $=$ SumStatsGroup1 out $=$ SumStatsTrGroup1 $($ rename $=($ Col1 $=Y))$; by Treatment Day_TYPE__FREQ_;

run;

Proc sort data=SumStatsTrGroup1;

by _LABEL_;

*Simple interaction plot for each response variable;

Proc Sgplot;

by _LABEL_;

series $\mathrm{X}=$ Treatment $\mathrm{Y}=\mathrm{Y} /$ group $=$ Day;

run;

proc corr data $=$ Group 1 plots $=$ scatter;

var treatment;

with TRP_Diff;

run;

proc corr data $=$ Group 1 plots $=$ scatter;

var treatment;

with RatioDiff;

run;

title 'Difference in serum free Trp';

Proc Mixed data=Group1;

class Treatment Day Horse;

model TRP_Diff $=$ Treatment $\mid$ Day $/ \mathrm{ddfm}=\mathrm{kr}$ solution residual ;

random Horse Horse*Treatment ;

slice Treatment*Day / sliceby $=$ Day pdiff;

run; 
title 'Difference in TRP:LNAA';

Proc Mixed data $=$ Group 1 ;

class Treatment Day Horse;

model RatioDiff $=$ Treatment $\mid$ Day $/ \mathrm{ddfm}=\mathrm{kr}$ solution residual ;

random Horse Horse*Treatment ;

slice Treatment*Day / sliceby $=$ Day pdiff;

run;

title 'Washout serum free Trp';

Proc Mixed data $=$ Group1;

where Day $=1$;

class Treatment TrtNum Day Horse;

model PreTRP $=$ TrtNum $/ \mathrm{ddfm}=\mathrm{kr}$ solution residual ;

random Horse Horse*TrtNum ;

lsmeans TrtNum/pdiff;

run;

title 'Washout serum TRP:LNAA';

Proc Mixed data $=$ Group1;

where Day $=1$;

class Treatment TrtNum Day Horse;

model PreRatio $=\mathrm{TrtNum} / \mathrm{ddfm}=\mathrm{kr}$ solution residual ;

random Horse Horse*TrtNum ;

lsmeans TrtNum/pdiff;

run;

PROC IMPORT OUT=WORK.AABCCompare

DATAFILE="/home/britdav/AABCCompare.xlsx"

DBMS=XLSX REPLACE;

GETNAMES=YES;

RUN;

Proc Mixed data $=\mathrm{AABCC}$ ompare;

class Treatment DBC Horse;

model $\mathrm{TRP}=\mathrm{DBC} / \mathrm{ddfm}=\mathrm{kr}$ solution residual ;

random Horse Horse*Treatment ;

lsmeans DBC / pdiff;

run;

Proc Mixed data=AABCCompare;

class Treatment DBC Horse;

model Ratio $=\mathrm{DBC} / \mathrm{ddfm}=\mathrm{kr}$ solution residual ;

random Horse Horse*Treatment ;

lsmeans DBC / pdiff;

run; 
APPENDIX 4

\section{SAS SUMMARY OUTPUT}

Day 1 and Day 3 Results

DAY 1

The MEANS Procedure

\begin{tabular}{|c|c|c|c|c|c|}
\hline Treatment & $\begin{array}{r}\mathrm{N} \\
\text { Obs }\end{array}$ & Variable & Label & Mean & Std Dev \\
\hline 0 & 11 & $\begin{array}{l}\text { ExitSpeed } \\
\text { HR30 } \\
\text { HRR Diff } \\
\text { TimetoBL } \\
\text { Gluififf } \\
\text { GluPostAvg } \\
\text { Lactiffif } \\
\text { LactPostAvg } \\
\text { CortDiff } \\
\text { CortPostAvg }\end{array}$ & $\begin{array}{l}\text { Exitspeed } \\
\text { HR30 } \\
\text { HRR Diff } \\
\text { TimetoBL } \\
\text { GGulifif } \\
\text { GluPostAvg } \\
\text { Lactiffif } \\
\text { LactPostAvg } \\
\text { CortDiff } \\
\text { CortPostAvg }\end{array}$ & \begin{tabular}{|r|}
5.1110000 \\
102.6363636 \\
65.945454545 \\
225.7000000 \\
-4.83888899 \\
84.2200000 \\
0.17303850 \\
1.1549550 \\
2.7914811 \\
13.8581452 \\
\end{tabular} & $\begin{array}{r}1.14858880 \\
19.0329857 \\
20.25551901 \\
96.9227069 \\
6.532274744 \\
8.7901207 \\
0.2849126 \\
0.3062569 \\
5.5027572 \\
10.9708711\end{array}$ \\
\hline 20 & 11 & $\begin{array}{l}\text { Exitspeed } \\
\text { HR30ed } \\
\text { HRRDiff } \\
\text { TimetoBL } \\
\text { Gluififf } \\
\text { GlupostAvg } \\
\text { Lactiffif } \\
\text { LactPostavg } \\
\text { CortDiff } \\
\text { CortPostAvg }\end{array}$ & $\begin{array}{l}\text { Exitspeed } \\
\text { HR30ed } \\
\text { HRR Diff } \\
\text { TimetoBL } \\
\text { Glubiff } \\
\text { GlupostAvg } \\
\text { Lactiffif } \\
\text { LactPostavg } \\
\text { CortDiff } \\
\text { CortPostAvg }\end{array}$ & \begin{tabular}{|r|}
4.65400000 \\
98.6000000 \\
55.40000000 \\
206.7777778 \\
-6.59000000 \\
82.0500000 \\
-0.1029833 \\
0.9701400 \\
1.09431838 \\
12.1232398 \\
\end{tabular} & $\begin{array}{r}1.7737982 \\
18.8455712 \\
12.2764454 \\
71.4086440 \\
4.32424222 \\
7.6745684 \\
0.2326554 \\
0.2181086 \\
3.3103723 \\
6.6245716\end{array}$ \\
\hline 40 & 11 & $\begin{array}{l}\text { Exitspeed } \\
\text { HR30 } \\
\text { HRR Diff } \\
\text { TimetoBL } \\
\text { Gluifif } \\
\text { GluPostAvg } \\
\text { Lactiff } \\
\text { LactPostAvg } \\
\text { CortDiff } \\
\text { CortPostAvg }\end{array}$ & $\begin{array}{l}\text { Exitspeed } \\
\text { HR30 } \\
\text { HRR Diff } \\
\text { TimetoBL } \\
\text { GGuliff } \\
\text { GluPostAvg } \\
\text { Lactiff } \\
\text { LactPostAvg } \\
\text { CortDiff } \\
\text { CortPostAvg }\end{array}$ & \begin{tabular}{|r|}
5.1036364 \\
103.4000000 \\
68.30000000 \\
246.5000000 \\
-4.4318182 \\
82.6000000 \\
0.2058545 \\
1.2467318 \\
-1.8598621 \\
11.9115737 \\
\end{tabular} & \begin{tabular}{|l}
1.5033248 \\
26.1074702 \\
26.7168278 \\
89.5547629 \\
7.1485559 \\
6.1487397 \\
0.3562690 \\
0.3483196 \\
3.60009979 \\
10.5414673
\end{tabular} \\
\hline 60 & 11 & $\begin{array}{l}\text { ExitSpeed } \\
\text { HR30 } \\
\text { HRR Diff } \\
\text { TimetoBL } \\
\text { Gludiff } \\
\text { GluPostAvg } \\
\text { Lactiff } \\
\text { LactPostAvg } \\
\text { CortDiff } \\
\text { CortPostAvg }\end{array}$ & 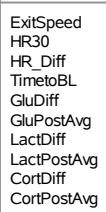 & \begin{tabular}{r|}
5.6044444 \\
159.90909090 \\
77.3636364 \\
215.00000000 \\
-0.37777778 \\
88.0888889 \\
0.2294000 \\
1.1278833 \\
-0.37770851 \\
12.6833565 \\
\end{tabular} & $\begin{array}{r}1.0521420 \\
24.4763337 \\
23.62371781 \\
64.4773862 \\
6.9744673 \\
8.5154557 \\
0.1388055 \\
0.2177054 \\
3.6004427 \\
12.6058338\end{array}$ \\
\hline
\end{tabular}

DAY 3

\begin{tabular}{|c|c|c|c|c|c|}
\hline \\
\hline Treatment & $\begin{array}{r}\mathrm{N} \\
\text { Obs }\end{array}$ & Variable & Label & Mean & Std Dev \\
\hline 0 & 11 & 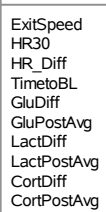 & $\begin{array}{l}\text { Exitspeed } \\
\text { HR30 } \\
\text { HR_Diff } \\
\text { TRimetoBL } \\
\text { Gluiff } \\
\text { GluPostAvg } \\
\text { Lactiff } \\
\text { LactPostAvg } \\
\text { Cortifif } \\
\text { CortPostAvg }\end{array}$ & $\begin{array}{r}5.20363643 \\
10.272727273 \\
72.8181818 \\
238.00000000 \\
-3.27500000 \\
84.7450000 \\
0.1782167 \\
1.1050667 \\
-0.3263981 \\
12.9971851\end{array}$ & $\begin{array}{r}1.34544090 \\
24.47390012 \\
29.1952574 \\
84.2531899 \\
4.1620808 \\
9.6280392 \\
0.2547027 \\
0.3313360 \\
6.34410143 \\
11.6014889\end{array}$ \\
\hline 20 & 11 & 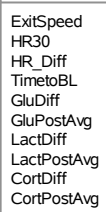 & $\begin{array}{l}\text { Exispeed } \\
\text { HR30 } \\
\text { HR_Diff } \\
\text { TimetoBL } \\
\text { GluLiff } \\
\text { GluPostAvg } \\
\text { Lactifif } \\
\text { LactPostAvg } \\
\text { CortDiff } \\
\text { CortPostAvg }\end{array}$ & $\begin{array}{r}5.5545455 \\
10.38181818 \\
67.7272727 \\
230.1000000 \\
1.50500000 \\
85.0045455 \\
0.29272333 \\
1.1849250 \\
0.3276037 \\
10.71998882\end{array}$ & $\begin{array}{r}1.3703311 \\
19.46639844 \\
18.35850550 \\
135.89000291 \\
8.6516451 \\
3.9880104 \\
0.37445439 \\
0.3126254 \\
3.4494645 \\
5.7296725\end{array}$ \\
\hline 40 & 11 & 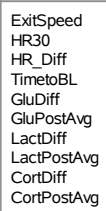 & $\begin{array}{l}\text { Exitspeed } \\
\text { HR30 } \\
\text { HR_Diff } \\
\text { TimetoBL } \\
\text { GluDiff } \\
\text { GluPostAvg } \\
\text { Lactifif } \\
\text { LactPostAvg } \\
\text { CortDiff } \\
\text { CortPostAvg }\end{array}$ & $\begin{array}{r}5.1318182 \\
99.7000000 \\
65.60000000 \\
318.1000000 \\
-2.57000000 \\
87.1727273 \\
0.228167 \\
1.24386767 \\
-0.1416802 \\
13.2783739\end{array}$ & 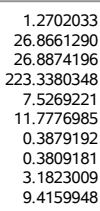 \\
\hline 60 & 11 & 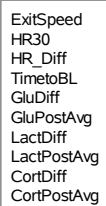 & $\begin{array}{l}\text { Exitspeed } \\
\text { HR30 } \\
\text { HR_Diff } \\
\text { TRimetoBL } \\
\text { Gludiff } \\
\text { GluPostAvg } \\
\text { Lactifif } \\
\text { LactPostAvg } \\
\text { CortDiff } \\
\text { CortPostAvg }\end{array}$ & 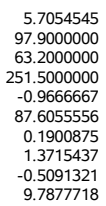 & 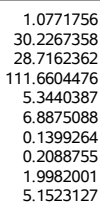 \\
\hline
\end{tabular}


Day 1 Exit Speed

The Mixed Procedure

\begin{tabular}{|l|r|r|r|r|}
\hline \multicolumn{5}{|c|}{ Type 3 Tests of Fixed Effects } \\
\hline Effect & $\begin{array}{r}\text { Num } \\
\text { DF }\end{array}$ & $\begin{array}{r}\text { Den } \\
\text { DF }\end{array}$ & F Value & Pr > F \\
\hline Group & 2 & 8.15 & 0.59 & 0.5786 \\
\hline Temperature & 1 & 25.2 & 21.47 & $<.0001$ \\
\hline Treatment & 3 & 25.3 & 1.25 & 0.3125 \\
\hline
\end{tabular}

\begin{tabular}{|l|c|r|r|r|r|r|}
\hline \multicolumn{7}{|c|}{ Least Squares Means Estimate } \\
\hline Effect & Label & Estimate & $\begin{array}{r}\text { Standard } \\
\text { Error }\end{array}$ & DF & t Value & Pr $>$ t| $\mid$ \\
\hline Treatment & Row 1 & -0.03741 & 0.2726 & 25.3 & -0.14 & 0.8919 \\
\hline
\end{tabular}

Least Squares Means

\begin{tabular}{|l|l|r|r|r|r|l|}
\hline \multicolumn{7}{|c|}{ Least Squares Means } \\
\hline Effect & Treatment & Estimate & $\begin{array}{r}\text { Standard } \\
\text { Error }\end{array}$ & DF & t Value & Pr $>\mid$ tt \\
\hline Treatment & 0 & 5.0861 & 0.3789 & 15 & 13.42 & $<.0001$ \\
\hline Treatment & 20 & 4.7708 & 0.3790 & 15 & 12.59 & $<.0001$ \\
\hline Treatment & 40 & 5.1639 & 0.3710 & 14 & 13.92 & $<.0001$ \\
\hline Treatment & 60 & 5.4357 & 0.3903 & 16.4 & 13.93 & $<.0001$ \\
\hline
\end{tabular}

Differences of Least Squares Means

\begin{tabular}{|l|l|l|r|r|r|r|r|}
\hline \multicolumn{7}{|c|}{ Differences of Least Squares Means } \\
\hline Effect & Treatment & Treatment & Estimate & $\begin{array}{r}\text { Standard } \\
\text { Error }\end{array}$ & DF & t Value & Pr $>$ |t 1 \\
\hline Treatment & 0 & 20 & 0.3153 & 0.3332 & 25.3 & 0.95 & 0.3530 \\
\hline Treatment & 0 & 40 & -0.07785 & 0.3230 & 25.2 & -0.24 & 0.8115 \\
\hline Treatment & 0 & 60 & -0.3496 & 0.3479 & 25.4 & -1.01 & 0.3243 \\
\hline Treatment & 20 & 40 & -0.3931 & 0.3229 & 25.2 & -1.22 & 0.2347 \\
\hline Treatment & 20 & 60 & -0.6649 & 0.3484 & 25.4 & -1.91 & 0.0677 \\
\hline Treatment & 40 & 60 & -0.2718 & 0.3388 & 25.3 & -0.80 & 0.4299 \\
\hline
\end{tabular}

Day 3 Exit Speed

The Mixed Procedure

\begin{tabular}{|l|r|r|r|r|}
\hline \multicolumn{5}{|c|}{ Type 3 Tests of Fixed Effects } \\
\hline Effect & $\begin{array}{r}\text { Num } \\
\text { DF }\end{array}$ & $\begin{array}{r}\text { Den } \\
\text { DF }\end{array}$ & F Value & Pr > F \\
\hline Group & 2 & 8.84 & 4.43 & 0.0465 \\
\hline Temperature & 1 & 29.3 & 0.00 & 0.9452 \\
\hline Treatment & 3 & 29 & 0.62 & 0.6048 \\
\hline
\end{tabular}

\begin{tabular}{|l|r|r|r|r|r|r|}
\hline \multicolumn{7}{|c|}{ Least Squares Means Estimate } \\
\hline Effect & Label & Estimate & $\begin{array}{r}\text { Standard } \\
\text { Error }\end{array}$ & DF & t Value & Pr $>$ tt \\
\hline Treatment & Row 1 & -0.2621 & 0.4013 & 29.01 & -0.65 & 0.5189 \\
\hline
\end{tabular}

Least Squares Mean

\begin{tabular}{|l|l|r|r|r|r|r|}
\hline \multicolumn{7}{|c|}{ Least Squares Means } \\
\hline Effect & Treatment & Estimate & $\begin{array}{r}\text { Standard } \\
\text { Error }\end{array}$ & DF & t Value & Pr $>$ tt \\
\hline Treatment & 0 & 5.1867 & 0.3550 & 36.1 & 14.61 & $<.0001$ \\
\hline Treatment & 20 & 5.5371 & 0.3556 & 36.2 & 15.57 & $<.0001$ \\
\hline Treatment & 40 & 5.1191 & 0.3559 & 36.2 & 14.38 & $<.0001$ \\
\hline Treatment & 60 & 5.6902 & 0.3541 & 36.1 & 16.07 & $<.0001$ \\
\hline
\end{tabular}

\begin{tabular}{|l|l|l|r|r|r|r|r|}
\hline \multicolumn{7}{|c|}{ Differences of Least Squares Means } \\
\hline Effect & Treatment & Treatment & Estimate & $\begin{array}{r}\text { Standard } \\
\text { Error }\end{array}$ & DF & t Value & Pr > |t| \\
\hline Treatment & 0 & 20 & -0.3503 & 0.4905 & 29 & -0.71 & 0.4808 \\
\hline Treatment & 0 & 40 & 0.06762 & 0.4942 & 29 & 0.14 & 0.8921 \\
\hline Treatment & 0 & 60 & -0.5034 & 0.4910 & 29 & -1.03 & 0.3137 \\
\hline Treatment & 20 & 40 & 0.4180 & 0.4953 & 29 & 0.84 & 0.4056 \\
\hline Treatment & 20 & 60 & -0.1531 & 0.4915 & 29 & -0.31 & 0.7577 \\
\hline Treatment & 40 & 60 & -0.5711 & 0.4919 & 29 & -1.16 & 0.2551 \\
\hline
\end{tabular}


Day 1 Exit Speed

The Mixed Procedure

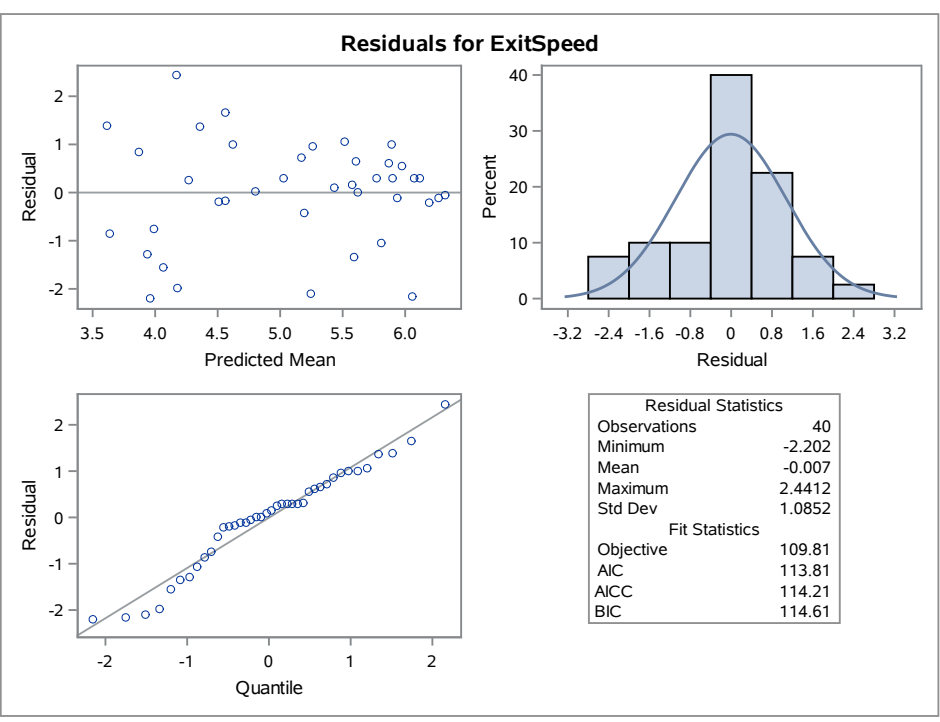

Day 3 Exit Speed

The Mixed Procedure

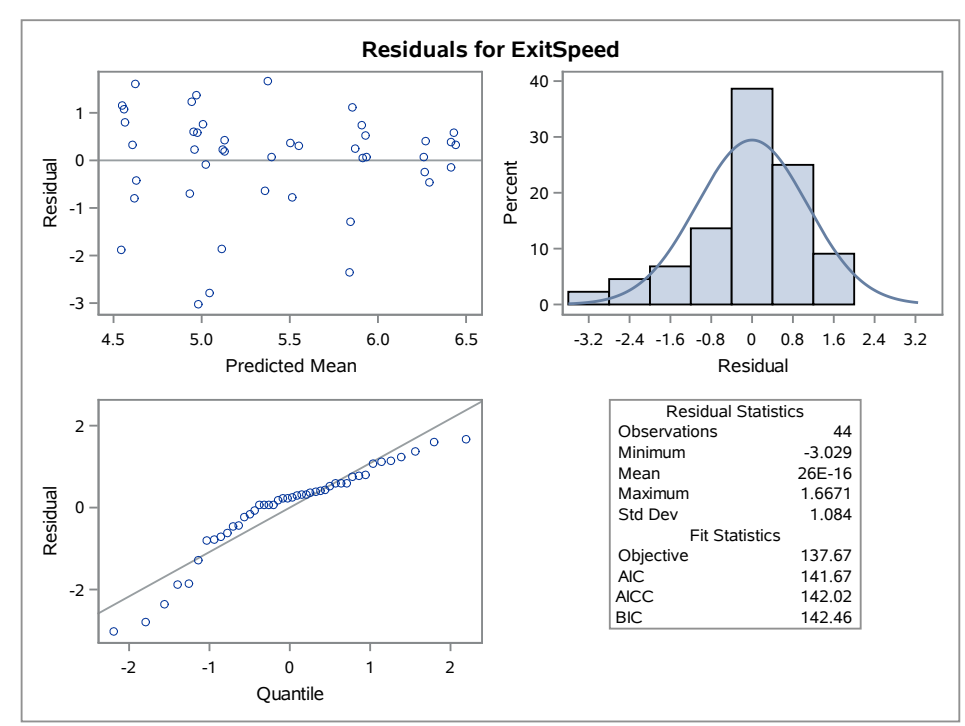


Day 1 HR 30

The Mixed Procedure

\begin{tabular}{|l|r|r|r|r|}
\hline \multicolumn{5}{|c|}{ Type 3 Tests of Fixed Effects } \\
\hline Effect & $\begin{array}{r}\text { Num } \\
\text { DF }\end{array}$ & $\begin{array}{r}\text { Den } \\
\text { DF }\end{array}$ & F Value & Pr > F \\
\hline TrtNum & 3 & 25.5 & 0.39 & 0.7613 \\
\hline Treatment & 3 & 25.5 & 0.89 & 0.4586 \\
\hline
\end{tabular}

\begin{tabular}{|l|l|r|r|r|r|r|}
\hline \multicolumn{7}{|c|}{ Least Squares Means Estimate } \\
\hline Effect & Label & Estimate & $\begin{array}{r}\text { Standard } \\
\text { Error }\end{array}$ & DF & t Value & Pr $>$ |t| \\
\hline Treatment & Row 1 & -0.9792 & 6.0371 & 25.33 & -0.16 & 0.8724 \\
\hline
\end{tabular}

\begin{tabular}{|l|l|r|r|r|r|l|}
\hline \multicolumn{7}{|c|}{ Least Squares Means } \\
\hline Effect & Treatment & Estimate & $\begin{array}{r}\text { Standard } \\
\text { Error }\end{array}$ & DF & t Value & Pr $>$ |t| \\
\hline Treatment & 0 & 102.83 & 6.7946 & 25 & 15.13 & $<.0001$ \\
\hline Treatment & 20 & 97.5991 & 7.0538 & 26.6 & 13.84 & $<.0001$ \\
\hline Treatment & 40 & 104.04 & 7.0556 & 26.6 & 14.75 & $<.0001$ \\
\hline Treatment & 60 & 109.78 & 6.7957 & 25 & 16.15 & $<.0001$ \\
\hline
\end{tabular}

\begin{tabular}{|l|l|l|r|r|r|r|r|}
\hline \multicolumn{7}{|c|}{ Differences of Least Squares Means } \\
\hline Effect & Treatment & Treatment & Estimate & $\begin{array}{r}\text { Standard } \\
\text { Error }\end{array}$ & DF & t Value & Pr $>$ It \\
\hline Treatment & 0 & 20 & 5.2280 & 7.5524 & 25.5 & 0.69 & 0.4950 \\
\hline Treatment & 0 & 40 & -1.2107 & 7.5530 & 25.5 & -0.16 & 0.8739 \\
\hline Treatment & 0 & 60 & -6.9549 & 7.2998 & 25.2 & -0.95 & 0.3497 \\
\hline Treatment & 20 & 40 & -6.4388 & 7.7839 & 25.8 & -0.83 & 0.4157 \\
\hline Treatment & 20 & 60 & -12.1830 & 7.5089 & 25.5 & -1.62 & 0.1170 \\
\hline Treatment & 40 & 60 & -5.7442 & 7.5578 & 25.5 & -0.76 & 0.4542 \\
\hline
\end{tabular}

Day 3 HR 30

The Mixed Procedure

\begin{tabular}{|l|r|r|r|r|}
\hline \multicolumn{7}{|c|}{ Type 3 Tests of Fixed Effects } \\
\hline Effect & $\begin{array}{r}\text { Num } \\
\text { DF }\end{array}$ & $\begin{array}{r}\text { Den } \\
\text { DF }\end{array}$ & F Value & Pr > F \\
\hline TrtNum & 3 & 25.4 & 2.08 & 0.1283 \\
\hline Treatment & 3 & 25.4 & 0.46 & 0.7112 \\
\hline
\end{tabular}

Least Squares Means Estimate

\begin{tabular}{|l|l|l|r|r|r|r|}
\hline Effect & Label & Estimate & $\begin{array}{r}\text { Standard } \\
\text { Error }\end{array}$ & DF & t Value & $\operatorname{Pr}>|t|$ \\
\hline
\end{tabular}

\begin{tabular}{|l|l|r|r|r|r|r|}
\hline Treatment & Row 1 & 7.4936 & 7.1308 & 25.22 & 1.05 & 0.3033 \\
\hline
\end{tabular}

\begin{tabular}{|l|l|r|r|r|r|l|}
\hline \multicolumn{7}{|c|}{ Least Squares Means } \\
\hline Effect & Treatment & Estimate & $\begin{array}{r}\text { Standard } \\
\text { Error }\end{array}$ & DF & t Value & Pr $>$ |t| \\
\hline Treatment & 0 & 108.36 & 7.4783 & 27.9 & 14.49 & $<.0001$ \\
\hline Treatment & 20 & 103.66 & 7.4811 & 27.9 & 13.86 & $<.0001$ \\
\hline Treatment & 40 & 99.9135 & 7.8013 & 29.4 & 12.81 & $<.0001$ \\
\hline Treatment & 60 & 99.0201 & 7.8013 & 29.4 & 12.69 & $<.0001$ \\
\hline
\end{tabular}

Differences of Least Squares Means

\begin{tabular}{|l|l|l|r|r|r|r|r|}
\hline \multicolumn{7}{|c|}{ Differences of Least Squares Means } \\
\hline Effect & Treatment & Treatment & Estimate & $\begin{array}{r}\text { Standard } \\
\text { Error }\end{array}$ & DF & t Value & Pr $>$ tt \\
\hline Treatment & 0 & 20 & 4.6956 & 8.6112 & 25.1 & 0.55 & 0.5904 \\
\hline Treatment & 0 & 40 & 8.4459 & 8.9035 & 25.4 & 0.95 & 0.3518 \\
\hline Treatment & 0 & 60 & 9.3393 & 8.9035 & 25.4 & 1.05 & 0.3041 \\
\hline Treatment & 20 & 40 & 3.7502 & 8.8516 & 25.4 & 0.42 & 0.6754 \\
\hline Treatment & 20 & 60 & 4.6437 & 8.8516 & 25.4 & 0.52 & 0.6044 \\
\hline Treatment & 40 & 60 & 0.8935 & 9.1519 & 25.7 & 0.10 & 0.9230 \\
\hline
\end{tabular}


Day 1 HR 30

The Mixed Procedure

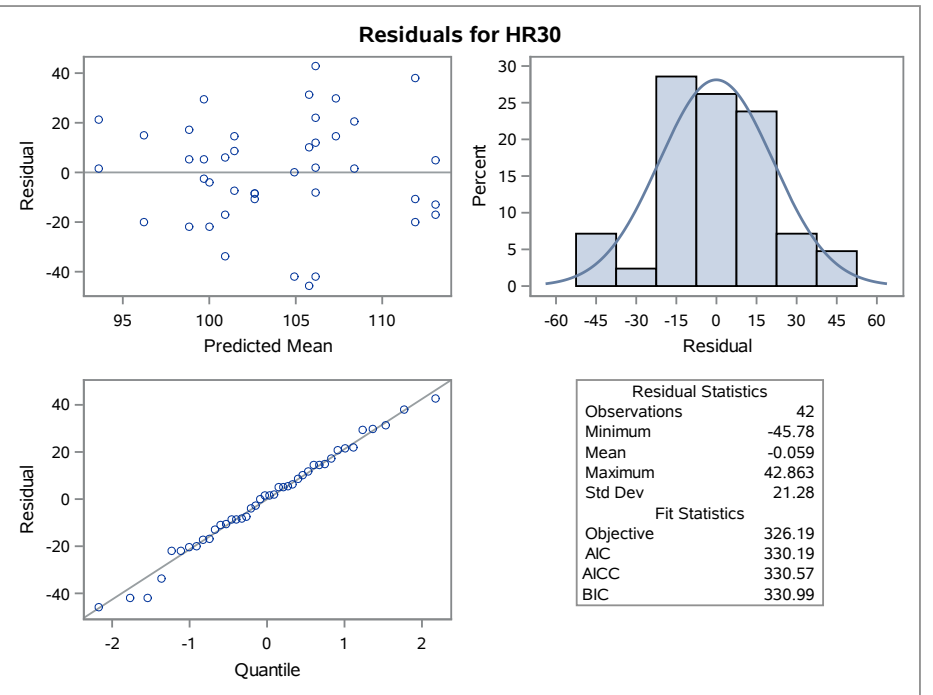

Day 3 HR 30

The Mixed Procedure

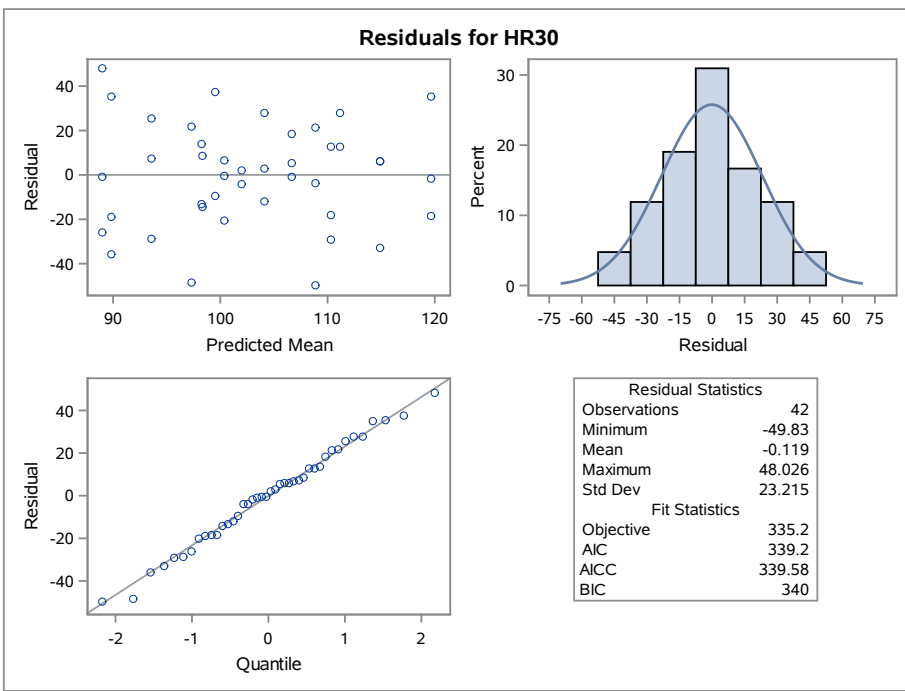


Day 1 HR Difference

\section{The Mixed Procedure}

\begin{tabular}{|l|r|r|r|r|}
\hline \multicolumn{5}{|c|}{ Type 3 Tests of Fixed Effects } \\
\hline Effect & $\begin{array}{r}\text { Num } \\
\text { DF }\end{array}$ & $\begin{array}{r}\text { Den } \\
\text { DF }\end{array}$ & F Value & Pr $>$ F \\
\hline Group & 2 & 8.09 & 0.89 & 0.4478 \\
\hline TrtNum & 3 & 25.3 & 1.17 & 0.3393 \\
\hline Treatment & 3 & 25.3 & 2.57 & 0.0762 \\
\hline
\end{tabular}

\begin{tabular}{|l|l|l|r|r|r|r|}
\hline Effect & Label & Estimate & $\begin{array}{r}\text { Standard } \\
\text { Error }\end{array}$ & DF & $\mathrm{t}$ Value & $\operatorname{Pr}>\mid \mathrm{tt}$ \\
\hline Trets
\end{tabular}

\begin{tabular}{l|l|l|l|l|l|l|}
\hline Treatment & Row 1 & 3.7407 & 5.7735 & 25.15 & 0.65 & 0.5229 \\
\hline
\end{tabular}

\begin{tabular}{|l|l|r|r|r|r|r|}
\hline \multicolumn{7}{|c|}{ Least Squares Means } \\
\hline Effect & Treatment & Estimate & $\begin{array}{r}\text { Standard } \\
\text { Error }\end{array}$ & DF & t Value & Pr $>$ |tt \\
\hline Treatment & 0 & 69.9473 & 6.4825 & 20.7 & 10.79 & $<.0001$ \\
\hline Treatment & 20 & 55.3262 & 6.7246 & 22.3 & 8.23 & $<.0001$ \\
\hline Treatment & 40 & 68.7876 & 6.7672 & 22.6 & 10.16 & $<.0001$ \\
\hline Treatment & 60 & 74.5059 & 6.4824 & 20.7 & 11.49 & $<.0001$ \\
\hline
\end{tabular}

\begin{tabular}{|l|l|l|r|r|r|r|r|}
\hline \multicolumn{7}{|c|}{ Differences of Least Squares Means } \\
\hline Effect & Treatment & Treatment & Estimate & $\begin{array}{r}\text { Standard } \\
\text { Error }\end{array}$ & DF & t Value & Pr $>$ It \\
\hline Treatment & 0 & 20 & 14.6211 & 7.2251 & 25.3 & 2.02 & 0.0537 \\
\hline Treatment & 0 & 40 & 1.1597 & 7.2269 & 25.3 & 0.16 & 0.8738 \\
\hline Treatment & 0 & 60 & -4.5586 & 6.9800 & 25.1 & -0.65 & 0.5196 \\
\hline Treatment & 20 & 40 & -13.4613 & 7.4537 & 25.5 & -1.81 & 0.0827 \\
\hline Treatment & 20 & 60 & -19.1797 & 7.1829 & 25.3 & -2.67 & 0.0131 \\
\hline Treatment & 40 & 60 & -5.7184 & 7.2319 & 25.3 & -0.79 & 0.4365 \\
\hline
\end{tabular}

Day 3 HR Difference

The Mixed Procedure

\begin{tabular}{|l|r|r|r|r|r|}
\multicolumn{7}{|c|}{ Type 3 Tests of Fixed Effects } \\
\hline Effect & $\begin{array}{r}\text { Num } \\
\text { DF }\end{array}$ & $\begin{array}{c}\text { Den } \\
\text { DF }\end{array}$ & F Value & Pr $>$ F \\
\hline Group & 2 & 8.1 & 4.93 & 0.0397 \\
\hline TrtNum & 3 & 25.1 & 4.03 & 0.0180 \\
\hline Treatment & 3 & 25.3 & 0.17 & 0.9131 \\
\hline
\end{tabular}

\begin{tabular}{|l|l|r|r|r|r|r|}
\hline \multicolumn{7}{|c|}{ Least Squares Means Estimate } \\
\hline Effect & Label & Estimate & $\begin{array}{r}\text { Standard } \\
\text { Error }\end{array}$ & DF & t Value & Pr $>$ |t| \\
\hline Treatment & Row 1 & 4.5217 & 7.3382 & 24.99 & 0.62 & 0.5433 \\
\hline
\end{tabular}

Least Squares Mean

\begin{tabular}{|l|l|r|r|r|r|r|}
\hline \multicolumn{7}{|c|}{ Least Squares Means } \\
\hline Effect & Treatment & Estimate & $\begin{array}{r}\text { Standard } \\
\text { Error }\end{array}$ & DF & t Value & Pr $>|t|$ \\
\hline Treatment & 0 & 71.8709 & 6.5172 & 31.9 & 11.03 & $<.0001$ \\
\hline Treatment & 20 & 68.5627 & 6.5299 & 31.9 & 10.50 & $<.0001$ \\
\hline Treatment & 40 & 68.1947 & 6.9445 & 32.4 & 9.82 & $<.0001$ \\
\hline Treatment & 60 & 65.2902 & 6.9445 & 32.4 & 9.40 & $<.0001$ \\
\hline
\end{tabular}

Differences of Least Squares Means

\begin{tabular}{|l|l|l|r|r|r|r|r|}
\hline \multicolumn{7}{|c|}{ Differences of Least Squares Means } \\
\hline Effect & Treatment & Treatment & Estimate & $\begin{array}{r}\text { Standard } \\
\text { Error }\end{array}$ & DF & t Value & Pr $>$ |tt \\
\hline Treatment & 0 & 20 & 3.3082 & 8.8597 & 24.9 & 0.37 & 0.7120 \\
\hline Treatment & 0 & 40 & 3.6762 & 9.1533 & 25.3 & 0.40 & 0.6913 \\
\hline Treatment & 0 & 60 & 6.5807 & 9.1533 & 25.3 & 0.72 & 0.4788 \\
\hline Treatment & 20 & 40 & 0.3680 & 9.0990 & 25.3 & 0.04 & 0.9681 \\
\hline Treatment & 20 & 60 & 3.2725 & 9.0990 & 25.3 & 0.36 & 0.7221 \\
\hline Treatment & 40 & 60 & 2.9045 & 9.3783 & 26 & 0.31 & 0.7593 \\
\hline
\end{tabular}


Day 1 HR Difference

The Mixed Procedure

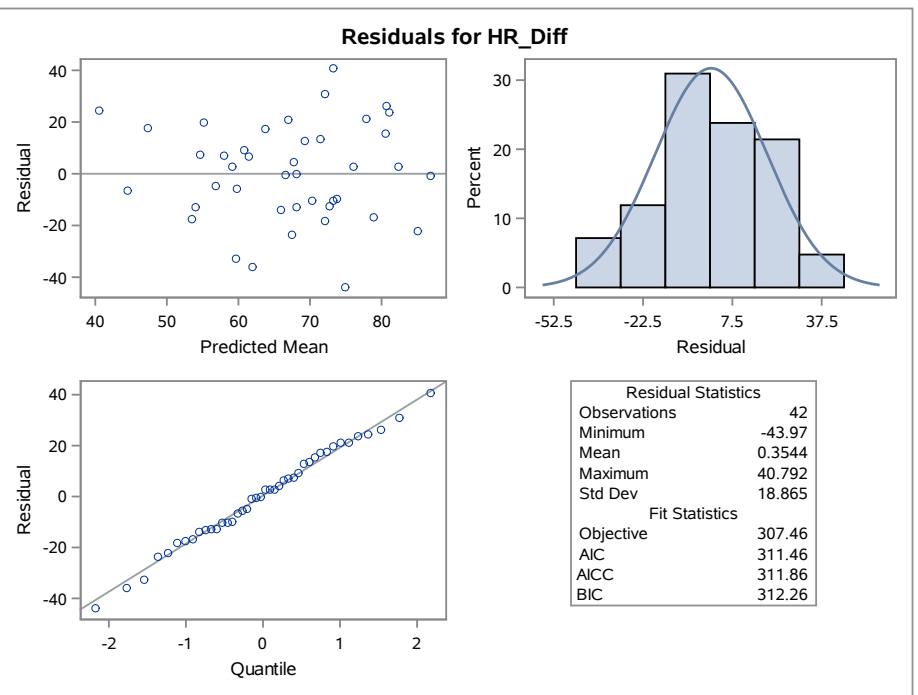

Day 3 HR Difference

The Mixed Procedure

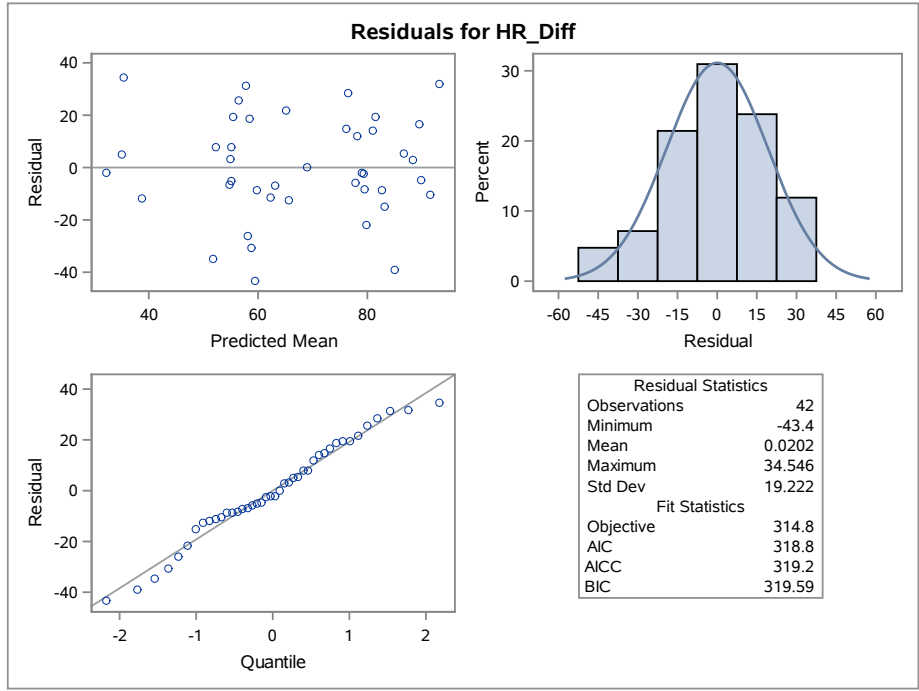


Day 1 Time to Return to Baseline

The Mixed Procedure

\begin{tabular}{|l|r|r|r|r|}
\hline \multicolumn{5}{|c|}{ Type 3 Tests of Fixed Effects } \\
\hline Effect & $\begin{array}{r}\text { Num } \\
\text { DF }\end{array}$ & $\begin{array}{r}\text { Den } \\
\text { DF }\end{array}$ & F Value & Pr > F \\
\hline Temperature & 1 & 27.5 & 6.73 & 0.0150 \\
\hline Treatment & 3 & 24.4 & 0.62 & 0.6060 \\
\hline
\end{tabular}

Least Squares Means Estimate

\begin{tabular}{|l|l|l|r|r|r|r|}
\hline Effect & Label & Estimate & $\begin{array}{r}\text { Standard } \\
\text { Error }\end{array}$ & DF & t Value & Pr $>|t|$ \\
\hline
\end{tabular}

\begin{tabular}{l|l|l|l|l|l|l|}
\hline Treatment & Row 1 & -0.2434 & 26.4636 & 24.65 & -0.01 & 0.9927 \\
\hline
\end{tabular}

\begin{tabular}{|l|l|r|r|r|r|l|}
\hline \multicolumn{7}{|c|}{ Least Squares Means } \\
\hline Effect & Treatment & Estimate & $\begin{array}{r}\text { Standard } \\
\text { Error }\end{array}$ & DF & t Value & Pr $>$ tt \\
\hline Treatment & 0 & 224.00 & 24.1456 & 33 & 9.28 & $<.0001$ \\
\hline Treatment & 20 & 213.53 & 25.5115 & 33.4 & 8.37 & $<.0001$ \\
\hline Treatment & 40 & 249.62 & 24.1412 & 33 & 10.34 & $<.0001$ \\
\hline Treatment & 60 & 209.59 & 24.1871 & 33 & 8.67 & $<.0001$ \\
\hline
\end{tabular}

\begin{tabular}{|l|l|l|r|r|r|r|r|}
\hline \multicolumn{7}{|c|}{ Differences of Least Squares Means } \\
\hline Effect & Treatment & Treatment & Estimate & $\begin{array}{r}\text { Standard } \\
\text { Error }\end{array}$ & DF & t Value & Pr > |t| \\
\hline Treatment & 0 & 20 & 10.4741 & 33.2647 & 25 & 0.31 & 0.7555 \\
\hline Treatment & 0 & 40 & -25.6135 & 32.1909 & 24.4 & -0.80 & 0.4339 \\
\hline Treatment & 0 & 60 & 14.4093 & 32.1574 & 24.2 & 0.45 & 0.6581 \\
\hline Treatment & 20 & 40 & -36.0875 & 32.8550 & 23.7 & -1.10 & 0.2831 \\
\hline Treatment & 20 & 60 & 3.9353 & 33.3017 & 25 & 0.12 & 0.9069 \\
\hline Treatment & 40 & 60 & 40.0228 & 32.2384 & 24.4 & 1.24 & 0.2262 \\
\hline
\end{tabular}

Day 3 Time to Return to Baseline

The Mixed Procedure

\begin{tabular}{|l|r|r|r|r|}
\hline \multicolumn{5}{|c|}{ Type 3 Tests of Fixed Effects } \\
\hline Effect & $\begin{array}{r}\text { Num } \\
\text { DF }\end{array}$ & $\begin{array}{r}\text { Den } \\
\text { DF }\end{array}$ & F Value & Pr > F \\
\hline Temperature & 1 & 29.7 & 18.83 & 0.0002 \\
\hline Treatment & 3 & 26.9 & 2.44 & 0.0858 \\
\hline
\end{tabular}

Least Squares Means Estimate

\begin{tabular}{|l|l|l|r|r|r|r|}
\hline \multicolumn{7}{|c|}{ Least Squares Means Estimate } \\
\hline Effect & Label & Estimate & $\begin{array}{r}\text { Standard } \\
\text { Error }\end{array}$ & DF & t Value & Pr $>$ |t| \\
\hline Treatment & Row 1 & -37.0710 & 33.4777 & 26.77 & -1.11 & 0.2780 \\
\hline
\end{tabular}

\begin{tabular}{|l|l|r|r|r|r|l|}
\hline \multicolumn{7}{|c|}{ Least Squares Means } \\
\hline Effect & Treatment & Estimate & $\begin{array}{r}\text { Standard } \\
\text { Error }\end{array}$ & DF & t Value & Pr $>$ tt \\
\hline Treatment & 0 & 228.69 & 38.2087 & 24.2 & 5.99 & $<.0001$ \\
\hline Treatment & 20 & 224.28 & 39.6891 & 25.7 & 5.65 & $<.0001$ \\
\hline Treatment & 40 & 325.88 & 39.5782 & 25.9 & 8.23 & $<.0001$ \\
\hline Treatment & 60 & 247.11 & 39.6722 & 25.7 & 6.23 & $<.0001$ \\
\hline
\end{tabular}

\begin{tabular}{|l|l|l|r|r|r|r|r|}
\hline \multicolumn{7}{|c|}{ Differences of Least Squares Means } \\
\hline Effect & Treatment & Treatment & Estimate & $\begin{array}{r}\text { Standard } \\
\text { Error }\end{array}$ & DF & t Value & Pr $>$ It \\
\hline Treatment & 0 & 20 & 4.4029 & 41.6165 & 26.9 & 0.11 & 0.9165 \\
\hline Treatment & 0 & 40 & -97.1953 & 41.6093 & 26.7 & -2.34 & 0.0273 \\
\hline Treatment & 0 & 60 & -18.4206 & 41.6083 & 26.9 & -0.44 & 0.6615 \\
\hline Treatment & 20 & 40 & -101.60 & 43.1177 & 27.3 & -2.36 & 0.0259 \\
\hline Treatment & 20 & 60 & -22.8235 & 42.2024 & 26.5 & -0.54 & 0.5932 \\
\hline Treatment & 40 & 60 & 78.7747 & 43.0877 & 27.3 & 1.83 & 0.0785 \\
\hline
\end{tabular}


Day 1 Time to Return to Baseline The Mixed Procedure

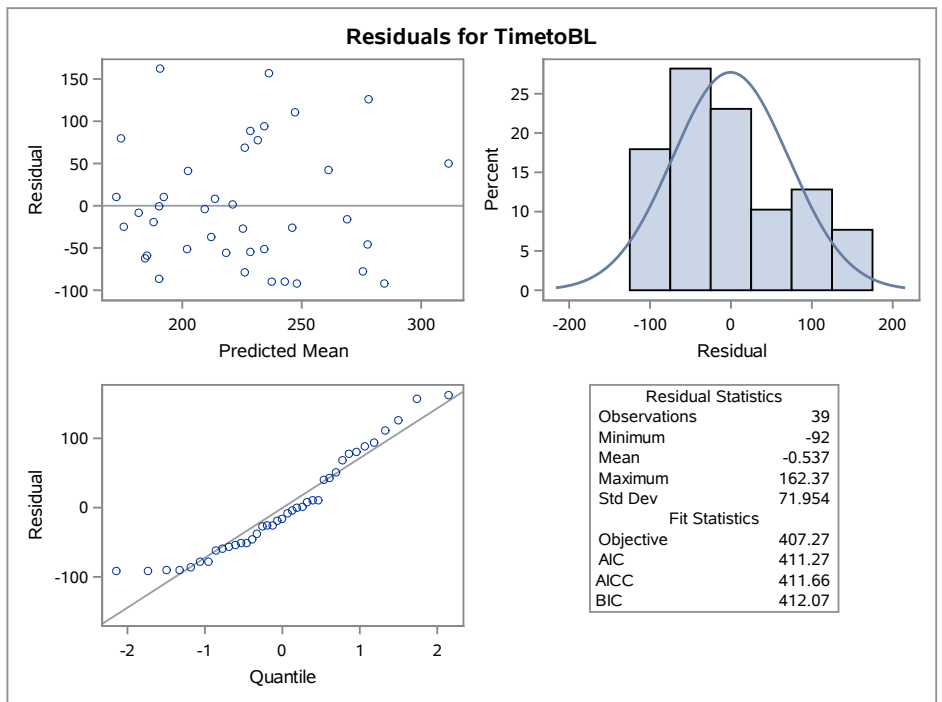

Day 3 Time to Return to Baseline The Mixed Procedure

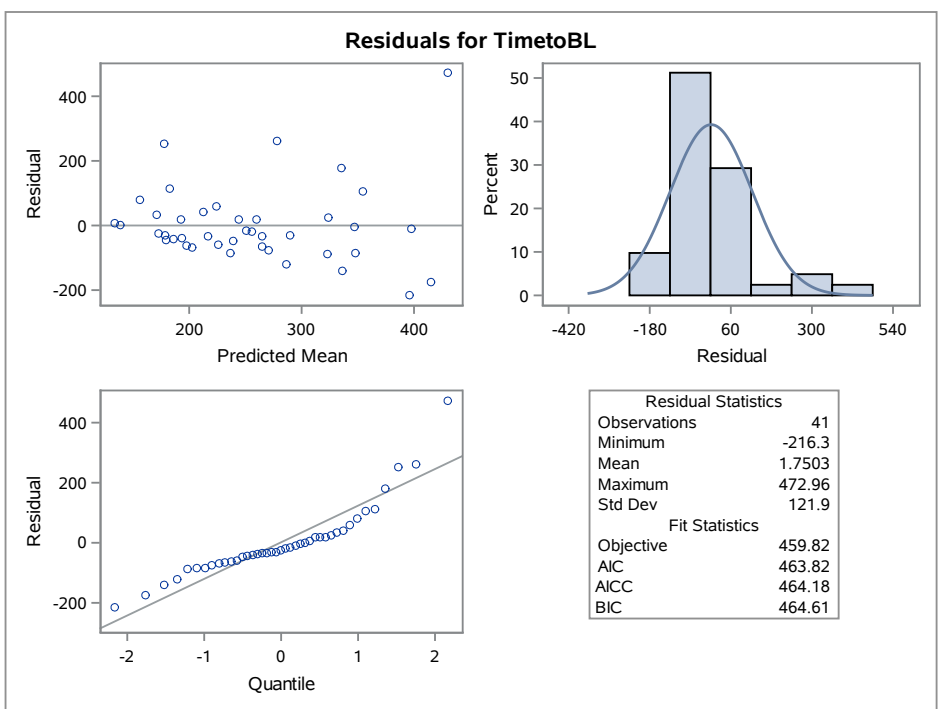

89 
Day 1 Glucose Difference

The Mixed Procedure

\begin{tabular}{|l|r|r|r|r|}
\hline \multicolumn{7}{|c|}{ Type 3 Tests of Fixed Effects } \\
\hline Effect & $\begin{array}{r}\text { Num } \\
\text { DF }\end{array}$ & $\begin{array}{r}\text { Den } \\
\text { DF }\end{array}$ & F Value & Pr > F \\
\hline GluRunDate & 8 & 20.6 & 1.36 & 0.2695 \\
\hline TimeLag & 1 & 24.9 & 2.30 & 0.1420 \\
\hline Treatment & 3 & 19 & 3.81 & 0.0271 \\
\hline
\end{tabular}

\begin{tabular}{|l|l|r|r|r|r|r|}
\hline \multicolumn{7}{|c|}{ Least Squares Means Estimate } \\
\hline Effect & Label & Estimate & $\begin{array}{r}\text { Standard } \\
\text { Error }\end{array}$ & DF & t Value & Pr $>|t|$ \\
\hline Treatment & Row 1 & 0.2812 & 2.1279 & 18.06 & 0.13 & 0.8963 \\
\hline
\end{tabular}

\begin{tabular}{|l|l|r|r|r|r|r|}
\hline \multicolumn{7}{|c|}{ Least Squares Means } \\
\hline Effect & Treatment & Estimate & $\begin{array}{r}\text { Standard } \\
\text { Error }\end{array}$ & DF & t Value & Pr $>$ t t 1 \\
\hline Treatment & 0 & -4.3400 & 1.9570 & 25.6 & -2.22 & 0.0357 \\
\hline Treatment & 20 & -8.2701 & 1.9689 & 25.2 & -4.20 & 0.0003 \\
\hline Treatment & 40 & -4.9574 & 2.0685 & 25.7 & -2.40 & 0.0241 \\
\hline Treatment & 60 & -0.6361 & 2.0332 & 25.4 & -0.31 & 0.7569 \\
\hline
\end{tabular}

\begin{tabular}{|l|l|l|r|r|r|r|r|}
\hline \multicolumn{7}{|c|}{ Differences of Least Squares Means } \\
\hline Effect & Treatment & Treatment & Estimate & $\begin{array}{r}\text { Standard } \\
\text { Error }\end{array}$ & DF & t Value & Pr > |t| \\
\hline Treatment & 0 & 20 & 3.9301 & 2.4298 & 18 & 1.62 & 0.1232 \\
\hline Treatment & 0 & 40 & 0.6174 & 2.7307 & 18.8 & 0.23 & 0.8236 \\
\hline Treatment & 0 & 60 & -3.7039 & 2.3985 & 18.7 & -1.54 & 0.1393 \\
\hline Treatment & 20 & 40 & -3.3127 & 2.2771 & 20 & -1.45 & 0.1613 \\
\hline Treatment & 20 & 60 & -7.6340 & 2.2601 & 18.1 & -3.38 & 0.0033 \\
\hline Treatment & 40 & 60 & -4.3213 & 2.5092 & 20.7 & -1.72 & 0.0999 \\
\hline
\end{tabular}

Day 3 Glucose Difference

\section{The Mixed Procedure}

\begin{tabular}{|l|r|r|r|r|}
\hline \multicolumn{5}{|c|}{ Type 3 Tests of Fixed Effects } \\
\hline Effect & $\begin{array}{r}\text { Num } \\
\text { DF }\end{array}$ & $\begin{array}{c}\text { Den } \\
\text { DF }\end{array}$ & F Value & Pr > F \\
\hline GluRunDate & 7 & 27 & 2.72 & 0.0284 \\
\hline TimeLag & 1 & 27 & 10.59 & 0.0031 \\
\hline Treatment & 3 & 27 & 1.17 & 0.3379 \\
\hline
\end{tabular}

\begin{tabular}{|l|r|r|r|r|r|r|}
\hline \multicolumn{7}{|c|}{ Least Squares Means Estimate } \\
\hline Effect & Label & Estimate & $\begin{array}{r}\text { Standard } \\
\text { Error }\end{array}$ & DF & t Value & Pr $>$ |t| \\
\hline Treatment & Row 1 & -1.9010 & 2.0013 & 27 & -0.95 & 0.3506 \\
\hline
\end{tabular}

Least Squares Means

\begin{tabular}{|l|l|r|r|r|r|r|}
\hline \multicolumn{7}{|c|}{ Least Squares Means } \\
\hline Effect & Treatment & Estimate & $\begin{array}{r}\text { Standard } \\
\text { Error }\end{array}$ & DF & t Value & Pr $>$ tt \\
\hline Treatment & 0 & -1.7820 & 1.7395 & 27 & -1.02 & 0.3147 \\
\hline Treatment & 20 & 1.7944 & 1.6904 & 27 & 1.06 & 0.2979 \\
\hline Treatment & 40 & -1.9823 & 1.7606 & 27 & -1.13 & 0.2701 \\
\hline Treatment & 60 & 0.5449 & 1.9034 & 27 & 0.29 & 0.7769 \\
\hline
\end{tabular}

Differences of Least Squares Means

\begin{tabular}{|l|l|l|r|r|r|r|r|}
\hline \multicolumn{7}{|c|}{ Differences of Least Squares Means } \\
\hline Effect & Treatment & Treatment & Estimate & $\begin{array}{r}\text { Standard } \\
\text { Error }\end{array}$ & DF & t Value & Pr $>$ |tt \\
\hline Treatment & 0 & 20 & -3.5764 & 2.4067 & 27 & -1.49 & 0.1489 \\
\hline Treatment & 0 & 40 & 0.2003 & 2.4302 & 27 & 0.08 & 0.9349 \\
\hline Treatment & 0 & 60 & -2.3269 & 2.5239 & 27 & -0.92 & 0.3647 \\
\hline Treatment & 20 & 40 & 3.7766 & 2.3988 & 27 & 1.57 & 0.1270 \\
\hline Treatment & 20 & 60 & 1.2495 & 2.5131 & 27 & 0.50 & 0.6231 \\
\hline Treatment & 40 & 60 & -2.5272 & 2.4676 & 27 & -1.02 & 0.3149 \\
\hline
\end{tabular}




\section{Day 1 Glucose Difference}

The Mixed Procedure

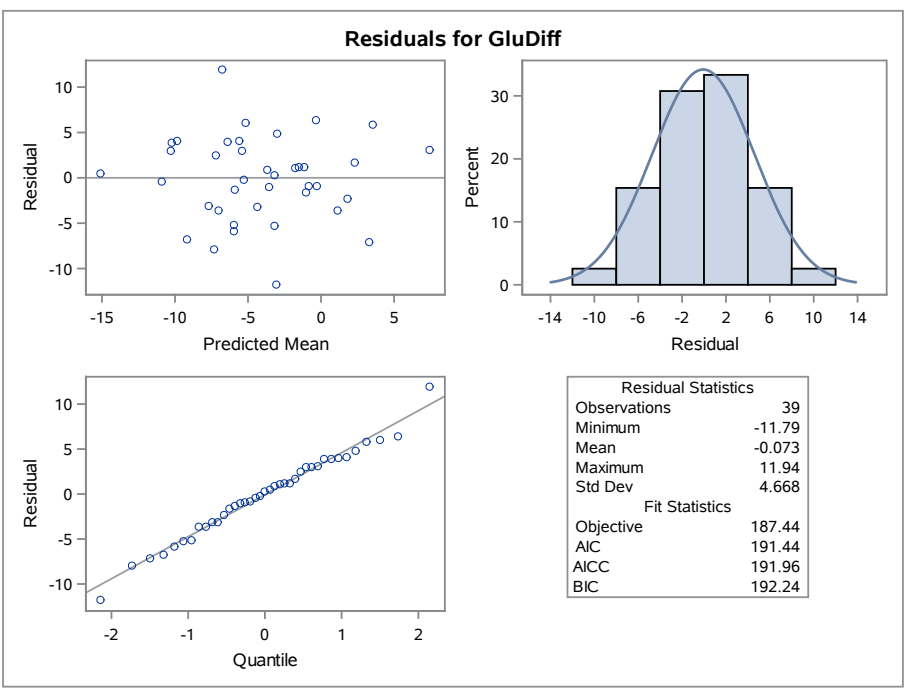

Day 3 Glucose Difference

The Mixed Procedure

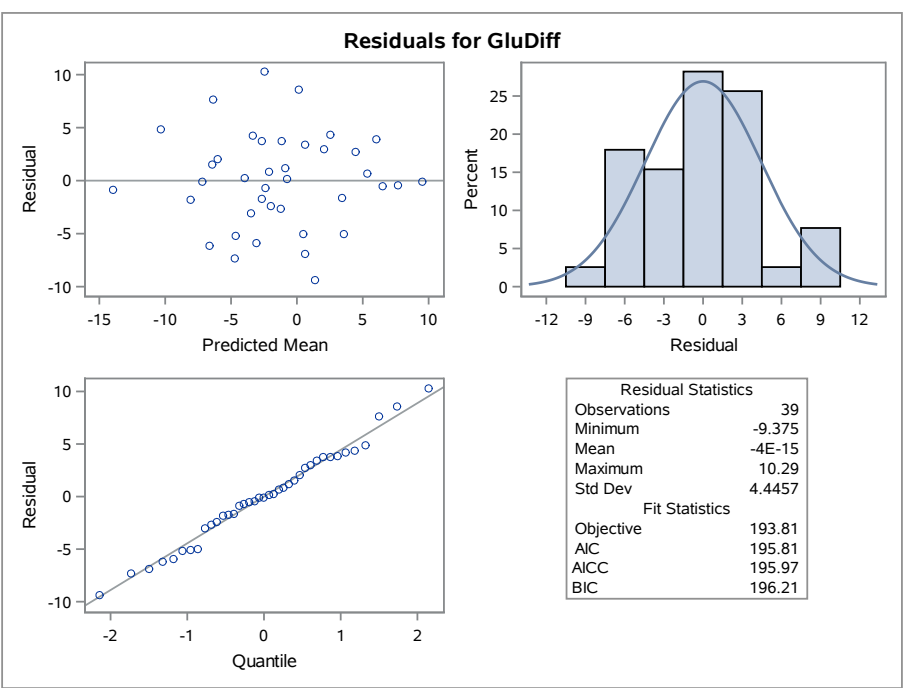




\section{Day 1 Glucose Post-Startle Averag}

The Mixed Procedure

\begin{tabular}{|l|r|r|r|r|}
\hline \multicolumn{5}{|c|}{ Type 3 Tests of Fixed Effects } \\
\hline Effect & $\begin{array}{r}\text { Num } \\
\text { DF }\end{array}$ & $\begin{array}{r}\text { Den } \\
\text { DF }\end{array}$ & F Value & Pr > F \\
\hline TimeLag & 1 & 36 & 6.39 & 0.0160 \\
\hline Treatment & 3 & 36 & 1.24 & 0.3082 \\
\hline
\end{tabular}

\begin{tabular}{|l|l|r|r|r|r|r|}
\hline \multicolumn{7}{|c|}{ Least Squares Means Estimate } \\
\hline Effect & Label & Estimate & $\begin{array}{r}\text { Standard } \\
\text { Error }\end{array}$ & DF & t Value & Pr $>|t|$ \\
\hline Treatment & Row 1 & -1.1391 & 2.6843 & 36 & -0.42 & 0.6738 \\
\hline
\end{tabular}

Least Squares Means

\begin{tabular}{|l|l|r|r|r|r|r|}
\hline \multicolumn{7}{|c|}{ Least Squares Means } \\
\hline Effect & Treatment & Estimate & $\begin{array}{r}\text { Standard } \\
\text { Error }\end{array}$ & DF & t Value & Pr $>$ |t $\mid$ \\
\hline Treatment & 0 & 83.3564 & 2.3252 & 36 & 35.85 & $<.0001$ \\
\hline Treatment & 20 & 81.7106 & 2.1971 & 36 & 37.19 & $<.0001$ \\
\hline Treatment & 40 & 83.8935 & 2.2518 & 36 & 37.26 & $<.0001$ \\
\hline Treatment & 60 & 87.8824 & 2.4258 & 36 & 36.23 & $<.0001$ \\
\hline
\end{tabular}

\begin{tabular}{|l|l|l|r|r|r|r|r|}
\hline \multicolumn{7}{|c|}{ Differences of Least Squares Means } \\
\hline Effect & Treatment & Treatment & Estimate & $\begin{array}{r}\text { Standard } \\
\text { Erro }\end{array}$ & DF & t Value & Pr $>$ |t| \\
\hline Treatment & 0 & 20 & 1.6458 & 3.1847 & 36 & 0.52 & 0.6085 \\
\hline Treatment & 0 & 40 & -0.5371 & 3.2904 & 36 & -0.16 & 0.8712 \\
\hline Treatment & 0 & 60 & -4.5260 & 3.3519 & 36 & -1.35 & 0.1854 \\
\hline Treatment & 20 & 40 & -2.1830 & 3.1678 & 36 & -0.69 & 0.4952 \\
\hline Treatment & 20 & 60 & -6.1718 & 3.2695 & 36 & -1.89 & 0.0671 \\
\hline Treatment & 40 & 60 & -3.9888 & 3.3225 & 36 & -1.20 & 0.2378 \\
\hline
\end{tabular}

\section{Day 3 Glucose Post-Startle Average}

\section{The Mixed Procedure}

$$
\begin{array}{|l|r|r|r|r|}
\hline \multicolumn{5}{|c|}{\text { Type } 3 \text { Tests of Fixed Effects }} \\
\hline \text { Effect } & \begin{array}{r}
\text { Num } \\
\text { DF }
\end{array} & \begin{array}{r}
\text { Den } \\
\text { DF }
\end{array} & \text { F Value } & \text { Pr > F } \\
\hline \text { TimeLag } & 1 & 34.8 & 5.86 & 0.0208 \\
\hline \text { Treatment } & 3 & 27.8 & 0.15 & 0.9320 \\
\hline
\end{array}
$$

\begin{tabular}{|l|l|r|r|r|r|r|}
\hline \multicolumn{7}{|c|}{ Least Squares Means Estimate } \\
\hline Effect & Label & Estimate & $\begin{array}{r}\text { Standard } \\
\text { Error }\end{array}$ & DF & t Value & Pr $>$ tt \\
\hline Treatment & Row 1 & -1.2672 & 2.9182 & 27.69 & -0.43 & 0.6675 \\
\hline
\end{tabular}

\begin{tabular}{|l|l|r|r|r|r|r|}
\hline \multicolumn{7}{|c|}{ Least Squares Means } \\
\hline Effect & Treatment & Estimate & $\begin{array}{r}\text { Standard } \\
\text { Error }\end{array}$ & DF & t Value & Pr $>$ |t $\mid$ \\
\hline Treatment & 0 & 85.1687 & 2.5740 & 35.9 & 33.09 & $<.0001$ \\
\hline Treatment & 20 & 85.4068 & 2.4460 & 35.9 & 34.92 & $<.0001$ \\
\hline Treatment & 40 & 86.8982 & 2.4430 & 35.9 & 35.57 & $<.0001$ \\
\hline Treatment & 60 & 87.0029 & 2.7334 & 36 & 31.83 & $<.0001$ \\
\hline
\end{tabular}

Differences of Least Squares Means

\begin{tabular}{|l|l|l|r|r|r|r|r|}
\hline \multicolumn{7}{|c|}{ Differences of Least Squares Means } \\
\hline Effect & Treatment & Treatment & Estimate & $\begin{array}{r}\text { Standard } \\
\text { Error }\end{array}$ & DF & t Value & Pr $>$ |t| \\
\hline Treatment & 0 & 20 & -0.2381 & 3.4900 & 27.5 & -0.07 & 0.9461 \\
\hline Treatment & 0 & 40 & -1.7295 & 3.5024 & 27.7 & -0.49 & 0.6253 \\
\hline Treatment & 0 & 60 & -1.8341 & 3.7018 & 28 & -0.50 & 0.6241 \\
\hline Treatment & 20 & 40 & -1.4914 & 3.4089 & 27 & -0.44 & 0.6652 \\
\hline Treatment & 20 & 60 & -1.5960 & 3.6304 & 28.5 & -0.44 & 0.6635 \\
\hline Treatment & 40 & 60 & -0.1046 & 3.6066 & 28.3 & -0.03 & 0.9771 \\
\hline
\end{tabular}




$$
\square
$$


Day 1 Lactate Difference

The Mixed Procedure

\begin{tabular}{|l|r|r|r|r|}
\hline \multicolumn{5}{|c|}{ Type 3 Tests of Fixed Effects } \\
\hline Effect & $\begin{array}{r}\text { Num } \\
\text { DF }\end{array}$ & $\begin{array}{r}\text { Den } \\
\text { DF }\end{array}$ & F Value & Pr $>$ F \\
\hline Treatment & 3 & 26.3 & 3.25 & 0.0376 \\
\hline
\end{tabular}

\begin{tabular}{|l|l|r|r|r|r|r|}
\hline \multicolumn{7}{|c|}{ Least Squares Means Estimate } \\
\hline Effect & Label & Estimate & $\begin{array}{r}\text { Standard } \\
\text { Error }\end{array}$ & DF & t Value & Pr $>\mid$ tt \\
\hline Treatment & Row 1 & 0.05637 & 0.09210 & 25.7 & 0.61 & 0.5459 \\
\hline
\end{tabular}

\begin{tabular}{|l|l|r|r|r|r|r|}
\hline \multicolumn{7}{|c|}{ Least Squares Means } \\
\hline Effect & Treatment & Estimate & $\begin{array}{r}\text { Standard } \\
\text { Error }\end{array}$ & DF & t Value & Pr $>$ tt \\
\hline Treatment & 0 & 0.1661 & 0.08612 & 33.2 & 1.93 & 0.0623 \\
\hline Treatment & 20 & -0.09890 & 0.09090 & 33.8 & -1.09 & 0.2843 \\
\hline Treatment & 40 & 0.2059 & 0.08197 & 32.9 & 2.51 & 0.0171 \\
\hline Treatment & 60 & 0.2224 & 0.09090 & 33.8 & 2.45 & 0.0198 \\
\hline
\end{tabular}

\begin{tabular}{|l|l|l|r|r|r|r|r|}
\hline \multicolumn{7}{|c|}{ Differences of Least Squares Means } \\
\hline Effect & Treatment & Treatment & Estimate & $\begin{array}{r}\text { Standard } \\
\text { Error }\end{array}$ & DF & t Value & Pr $>$ It \\
\hline Treatment & 0 & 20 & 0.2650 & 0.1156 & 25.9 & 2.29 & 0.0302 \\
\hline Treatment & 0 & 40 & -0.03972 & 0.1099 & 26.2 & -0.36 & 0.7207 \\
\hline Treatment & 0 & 60 & -0.05622 & 0.1156 & 25.9 & -0.49 & 0.6309 \\
\hline Treatment & 20 & 40 & -0.3048 & 0.1137 & 26.7 & -2.68 & 0.0124 \\
\hline Treatment & 20 & 60 & -0.3213 & 0.1194 & 26.5 & -2.69 & 0.0122 \\
\hline Treatment & 40 & 60 & -0.01650 & 0.1137 & 26.7 & -0.15 & 0.8857 \\
\hline
\end{tabular}

\section{Day 3 Lactate Difference}

The Mixed Procedure

\begin{tabular}{|l|r|r|r|r|}
\hline \multicolumn{5}{|c|}{ Type 3 Tests of Fixed Effects } \\
\hline Effect & $\begin{array}{r}\text { Num } \\
\text { DF }\end{array}$ & $\begin{array}{r}\text { Den } \\
\text { DF }\end{array}$ & F Value & Pr $>$ F \\
\hline Treatment & 3 & 23.8 & 0.39 & 0.7643 \\
\hline
\end{tabular}

\begin{tabular}{|c|c|c|c|c|c|c|}
\hline \multicolumn{7}{|c|}{ Least Squares Means Estimate } \\
\hline Effect & Label & Estimate & $\begin{array}{r}\text { Standard } \\
\text { Error }\end{array}$ & DF & t Value & $\operatorname{Pr}>|t|$ \\
\hline Treatment & Row 1 & -0.07351 & 0.1079 & 23.65 & -0.68 & 0.5024 \\
\hline
\end{tabular}

\begin{tabular}{|l|l|r|r|r|r|r|}
\hline \multicolumn{7}{|c|}{ Least Squares Means } \\
\hline Effect & Treatment & Estimate & $\begin{array}{r}\text { Standard } \\
\text { Error }\end{array}$ & DF & t Value & Pr $>$ tt \\
\hline Treatment & 0 & 0.1691 & 0.1029 & 29.1 & 1.64 & 0.1111 \\
\hline Treatment & 20 & 0.2994 & 0.1029 & 29.1 & 2.91 & 0.0069 \\
\hline Treatment & 40 & 0.2391 & 0.1029 & 29.1 & 2.32 & 0.0273 \\
\hline Treatment & 60 & 0.1892 & 0.1090 & 29.8 & 1.74 & 0.0931 \\
\hline
\end{tabular}

Differences of Least Squares Means

\begin{tabular}{|l|l|l|r|r|r|r|r|}
\hline \multicolumn{7}{|c|}{ Differences of Least Squares Means } \\
\hline Effect & Treatment & Treatment & Estimate & $\begin{array}{r}\text { Standard } \\
\text { Error }\end{array}$ & DF & t Value & Pr $>$ tt \\
\hline Treatment & 0 & 20 & -0.1304 & 0.1313 & 23.6 & -0.99 & 0.3308 \\
\hline Treatment & 0 & 40 & -0.07003 & 0.1313 & 23.6 & -0.53 & 0.5988 \\
\hline Treatment & 0 & 60 & -0.02012 & 0.1364 & 24.1 & -0.15 & 0.8840 \\
\hline Treatment & 20 & 40 & 0.06036 & 0.1313 & 23.6 & 0.46 & 0.6500 \\
\hline Treatment & 20 & 60 & 0.1103 & 0.1364 & 24.1 & 0.81 & 0.4268 \\
\hline Treatment & 40 & 60 & 0.04992 & 0.1364 & 24.1 & 0.37 & 0.7176 \\
\hline
\end{tabular}




\section{Day 1 Lactate Difference}

The Mixed Procedure

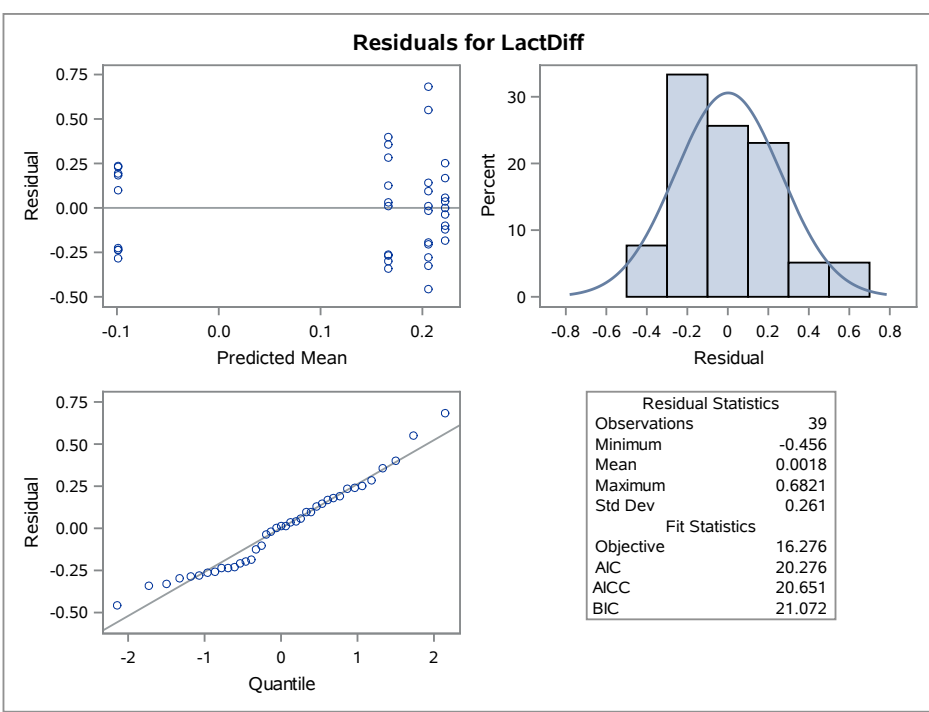

Day 3 Lactate Difference

The Mixed Procedure

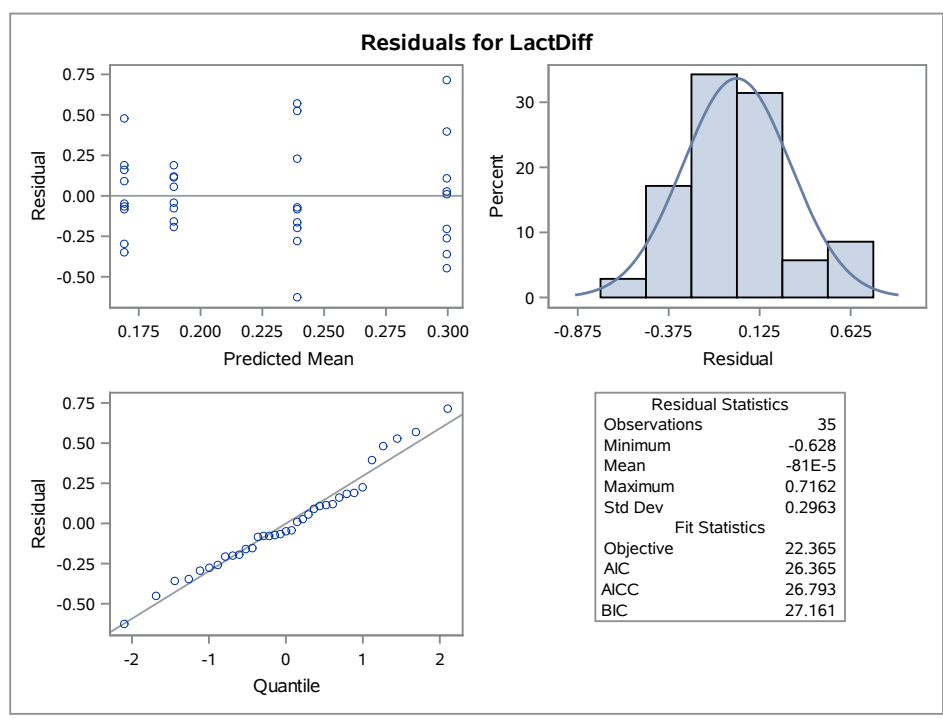


Day 1 Lactate Post-Startle Average

The Mixed Procedure

$$
\begin{array}{|l|r|r|r|r|}
\hline \multicolumn{5}{|c|}{\text { Type } 3 \text { Tests of Fixed Effects }} \\
\hline \text { Effect } & \begin{array}{r}
\text { Num } \\
\text { DF }
\end{array} & \begin{array}{r}
\text { Den } \\
\text { DF }
\end{array} & \text { F Value } & \text { Pr }>\text { F } \\
\hline \text { Treatment } & 3 & 26 & 2.16 & 0.1167 \\
\hline
\end{array}
$$

\begin{tabular}{|l|l|r|r|r|r|r|}
\hline \multicolumn{7}{|c|}{ Least Squares Means Estimate } \\
\hline Effect & Label & Estimate & $\begin{array}{r}\text { Standard } \\
\text { Error }\end{array}$ & DF & t Value & Pr $>$ |t| \\
\hline Treatment & Row 1 1 & 0.02538 & 0.08783 & 25.73 & 0.29 & 0.7749 \\
\hline
\end{tabular}

\begin{tabular}{|l|l|r|r|r|r|l|}
\hline \multicolumn{7}{|c|}{ Least Squares Means } \\
\hline Effect & Treatment & Estimate & $\begin{array}{r}\text { Standard } \\
\text { Error }\end{array}$ & DF & t Value & Pr $>$ tt \\
\hline Treatment & 0 & 1.1373 & 0.08949 & 30 & 12.71 & $<.0001$ \\
\hline Treatment & 20 & 0.9811 & 0.08932 & 30.1 & 10.98 & $<.0001$ \\
\hline Treatment & 40 & 1.2467 & 0.08557 & 28.6 & 14.57 & $<.0001$ \\
\hline Treatment & 60 & 1.1079 & 0.09386 & 31.5 & 11.80 & $<.0001$ \\
\hline
\end{tabular}

Differences of Least Squares Means

\begin{tabular}{|l|l|l|r|r|r|r|r|}
\hline \multicolumn{7}{|c|}{ Differences of Least Squares Means } \\
\hline Effect & Treatment & Treatment & Estimate & $\begin{array}{r}\text { Standard } \\
\text { Error }\end{array}$ & DF & t Value & Pr $>$ |t| \\
\hline Treatment & 0 & 20 & 0.1562 & 0.1083 & 26.2 & 1.44 & 0.1611 \\
\hline Treatment & 0 & 40 & -0.1095 & 0.1050 & 25.8 & -1.04 & 0.3071 \\
\hline Treatment & 0 & 60 & 0.02942 & 0.1104 & 25.6 & 0.27 & 0.7920 \\
\hline Treatment & 20 & 40 & -0.2656 & 0.1049 & 25.6 & -2.53 & 0.0178 \\
\hline Treatment & 20 & 60 & -0.1268 & 0.1121 & 26.7 & -1.13 & 0.2683 \\
\hline Treatment & 40 & 60 & 0.1389 & 0.1088 & 26.2 & 1.28 & 0.2130 \\
\hline
\end{tabular}

\section{Day 3 Lactate Post-Startle Average}

The Mixed Procedure

$$
\begin{array}{|l|r|r|r|r|}
\hline \multicolumn{5}{|c|}{\text { Type } 3 \text { Tests of Fixed Effects }} \\
\hline \text { Effect } & \begin{array}{r}
\text { Num } \\
\text { DF }
\end{array} & \begin{array}{r}
\text { Den } \\
\text { DF }
\end{array} & \text { F Value } & \text { Pr }>\text { F } \\
\hline \text { Treatment } & 3 & 23.6 & 1.35 & 0.2821 \\
\hline
\end{array}
$$

\begin{tabular}{|l|r|r|r|r|r|r|}
\hline \multicolumn{7}{|c|}{ Least Squares Means Estimate } \\
\hline Effect & Label & Estimate & $\begin{array}{r}\text { Standard } \\
\text { Error }\end{array}$ & DF & t Value & Pr $>|t| t \mid$ \\
\hline Treatment & Row 1 & -0.1690 & 0.1059 & 23.16 & -1.60 & 0.1241 \\
\hline
\end{tabular}

\begin{tabular}{|l|l|r|r|r|r|r|}
\hline \multicolumn{7}{|c|}{ Least Squares Means } \\
\hline Effect & Treatment & Estimate & $\begin{array}{r}\text { Standard } \\
\text { Error }\end{array}$ & DF & t Value & Pr $>$ |t $\mid$ \\
\hline Treatment & 0 & 1.0895 & 0.1055 & 28.9 & 10.33 & $<.0001$ \\
\hline Treatment & 20 & 1.1771 & 0.1001 & 28.1 & 11.76 & $<.0001$ \\
\hline Treatment & 40 & 1.2497 & 0.1055 & 28.9 & 11.85 & $<.0001$ \\
\hline Treatment & 60 & 1.3487 & 0.1114 & 30 & 12.11 & $<.0001$ \\
\hline
\end{tabular}

Differences of Least Squares Mean

\begin{tabular}{|l|l|l|r|r|r|r|r|}
\hline \multicolumn{7}{|c|}{ Differences of Least Squares Means } \\
\hline Effect & Treatment & Treatment & Estimate & $\begin{array}{r}\text { Standard } \\
\text { Error }\end{array}$ & DF & t Value & Pr $>$ |t| \\
\hline Treatment & 0 & 20 & -0.08764 & 0.1271 & 23.8 & -0.69 & 0.4972 \\
\hline Treatment & 0 & 40 & -0.1602 & 0.1292 & 22.9 & -1.24 & 0.2275 \\
\hline Treatment & 0 & 60 & -0.2592 & 0.1343 & 23.4 & -1.93 & 0.0659 \\
\hline Treatment & 20 & 40 & -0.07256 & 0.1271 & 23.8 & -0.57 & 0.5734 \\
\hline Treatment & 20 & 60 & -0.1716 & 0.1323 & 24.2 & -1.30 & 0.2069 \\
\hline Treatment & 40 & 60 & -0.09902 & 0.1343 & 23.4 & -0.74 & 0.4684 \\
\hline
\end{tabular}


Day 1 Lactate Post-Startle Average

\section{The Mixed Procedure}
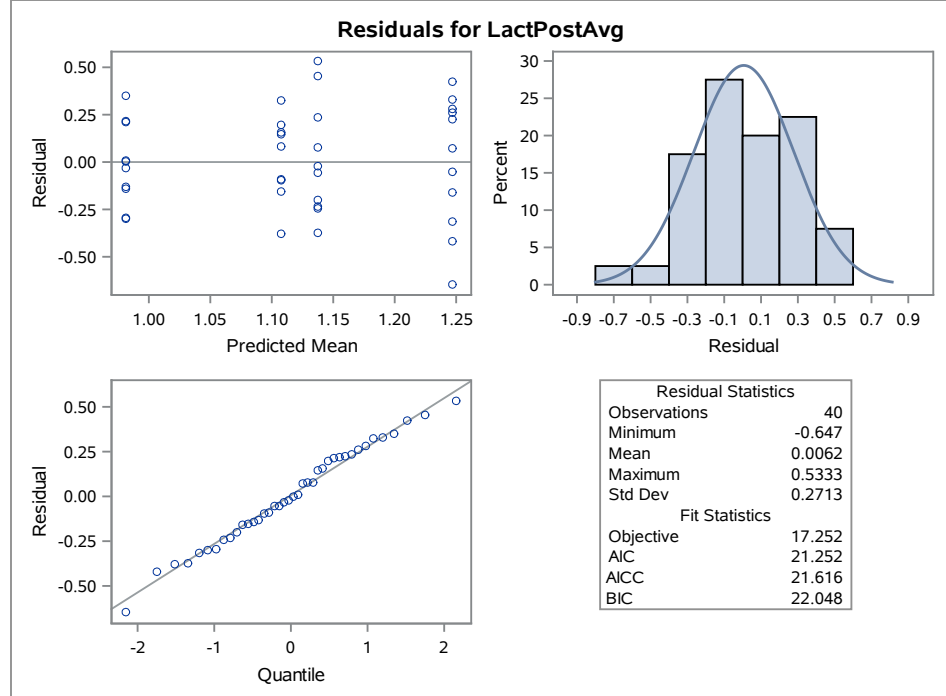

Day 3 Lactate Post-Startle Average

The Mixed Procedure

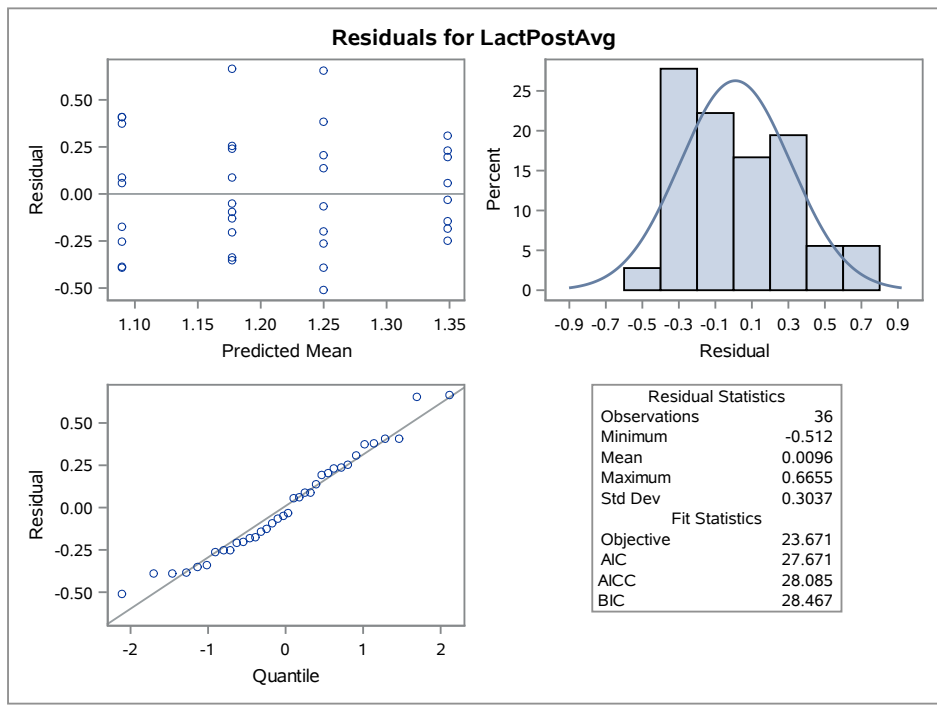


Day 1 Cortisol Difference

The Mixed Procedure

\begin{tabular}{|l|r|r|r|r|}
\hline \multicolumn{5}{|c|}{ Type 3 Tests of Fixed Effects } \\
\hline Effect & $\begin{array}{r}\text { Num } \\
\text { DF }\end{array}$ & $\begin{array}{r}\text { Den } \\
\text { DF }\end{array}$ & F Value & Pr $>$ F \\
\hline Treatment & 3 & 36 & 2.53 & 0.0722 \\
\hline
\end{tabular}

\begin{tabular}{|l|l|r|r|r|r|r|}
\hline \multicolumn{7}{|c|}{ Least Squares Means Estimate } \\
\hline Effect & Label & Estimate & $\begin{array}{r}\text { Standard } \\
\text { Error }\end{array}$ & DF & t Value & Pr $>$ |t| \\
\hline Treatment & Row 1 & 3.1721 & 1.4733 & 36 & 2.15 & 0.0381 \\
\hline
\end{tabular}

\begin{tabular}{|l|l|r|r|r|r|r|}
\hline \multicolumn{7}{|c|}{ Least Squares Means } \\
\hline Effect & Treatment & Estimate & $\begin{array}{r}\text { Standard } \\
\text { Error }\end{array}$ & DF & t Value & Pr $>$ |tt \\
\hline Treatment & 0 & 2.7915 & 1.2748 & 36 & 2.19 & 0.0351 \\
\hline Treatment & 20 & 1.0943 & 1.2748 & 36 & 0.86 & 0.3963 \\
\hline Treatment & 40 & -1.8590 & 1.2155 & 36 & -1.53 & 0.1349 \\
\hline Treatment & 60 & -0.3771 & 1.3438 & 36 & -0.28 & 0.7806 \\
\hline
\end{tabular}

\begin{tabular}{|l|l|l|r|r|r|r|r|}
\hline \multicolumn{7}{|c|}{ Differences of Least Squares Means } \\
\hline Effect & Treatment & Treatment & Estimate & $\begin{array}{r}\text { Standard } \\
\text { Error }\end{array}$ & DF & t Value & Pr $>\mid$ tt \\
\hline Treatment & 0 & 20 & 1.6972 & 1.8029 & 36 & 0.94 & 0.3528 \\
\hline Treatment & 0 & 40 & 4.6504 & 1.7614 & 36 & 2.64 & 0.0122 \\
\hline Treatment & 0 & 60 & 3.1686 & 1.8523 & 36 & 1.71 & 0.0958 \\
\hline Treatment & 20 & 40 & 2.9533 & 1.7614 & 36 & 1.68 & 0.1023 \\
\hline Treatment & 20 & 60 & 1.4714 & 1.8523 & 36 & 0.79 & 0.4322 \\
\hline Treatment & 40 & 60 & -1.4819 & 1.8120 & 36 & -0.82 & 0.4188 \\
\hline
\end{tabular}

Day 3 Cortisol Difference

The Mixed Procedure

Type 3 Tests of Fixed Effects

$$
\begin{array}{|l|r|r|r|r|}
\hline \text { Effect } & \begin{array}{r}
\text { Num } \\
\text { DF }
\end{array} & \begin{array}{c}
\text { Den } \\
\text { DF }
\end{array} & \text { F Value } & \text { Pr }>\text { F } \\
\hline \text { Treatment } & 3 & 24.1 & 0.06 & 0.9783 \\
\hline
\end{array}
$$

\begin{tabular}{|l|l|r|r|r|r|r|}
\hline \multicolumn{7}{|c|}{ Least Squares Means Estimate } \\
\hline Effect & Label & Estimate & $\begin{array}{r}\text { Standard } \\
\text { Error }\end{array}$ & DF & t Value & Pr $>\mid$ t| \\
\hline Treatment & Row 1 & -0.2446 & 1.4604 & 24.17 & -0.17 & 0.8684 \\
\hline
\end{tabular}

\begin{tabular}{|l|l|r|r|r|r|r|}
\hline \multicolumn{7}{|c|}{ Least Squares Means } \\
\hline Effect & Treatment & Estimate & $\begin{array}{r}\text { Standard } \\
\text { Error }\end{array}$ & DF & t Value & Pr $>$ |t| \\
\hline Treatment & 0 & -0.2864 & 1.3037 & 35.6 & -0.22 & 0.8274 \\
\hline Treatment & 20 & 0.3276 & 1.2364 & 35.4 & 0.26 & 0.7926 \\
\hline Treatment & 40 & -0.06896 & 1.3037 & 35.6 & -0.05 & 0.9581 \\
\hline Treatment & 60 & -0.3839 & 1.3820 & 35.7 & -0.28 & 0.7828 \\
\hline
\end{tabular}

Differences of Least Squares Means

\begin{tabular}{|l|l|l|r|r|r|r|r|}
\hline \multicolumn{7}{|c|}{ Differences of Least Squares Means } \\
\hline Effect & Treatment & Treatment & Estimate & $\begin{array}{r}\text { Standard } \\
\text { Error }\end{array}$ & DF & t Value & Pr $>$ t t \\
\hline Treatment & 0 & 20 & -0.6140 & 1.7430 & 23.8 & -0.35 & 0.7278 \\
\hline Treatment & 0 & 40 & -0.2174 & 1.7926 & 24.7 & -0.12 & 0.9045 \\
\hline Treatment & 0 & 60 & 0.09749 & 1.8339 & 23.9 & 0.05 & 0.9580 \\
\hline Treatment & 20 & 40 & 0.3966 & 1.7430 & 23.8 & 0.23 & 0.8220 \\
\hline Treatment & 20 & 60 & 0.7115 & 1.8023 & 24.7 & 0.39 & 0.6964 \\
\hline Treatment & 40 & 60 & 0.3149 & 1.8339 & 23.9 & 0.17 & 0.8651 \\
\hline
\end{tabular}


Day 1 Cortisol Difference

The Mixed Procedure

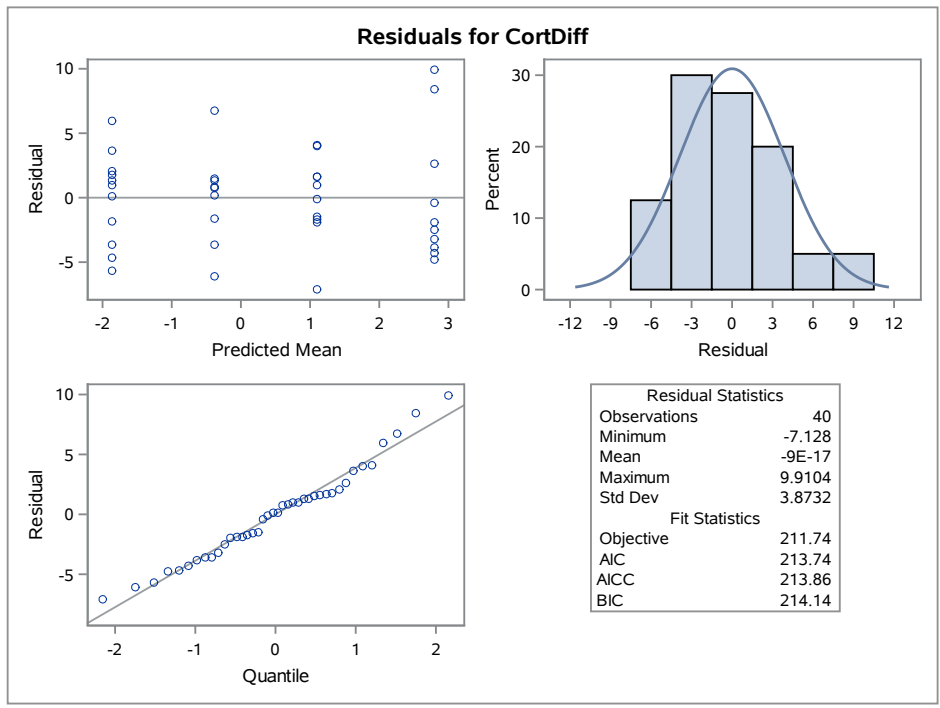

Day 3 Cortisol Difference

The Mixed Procedure

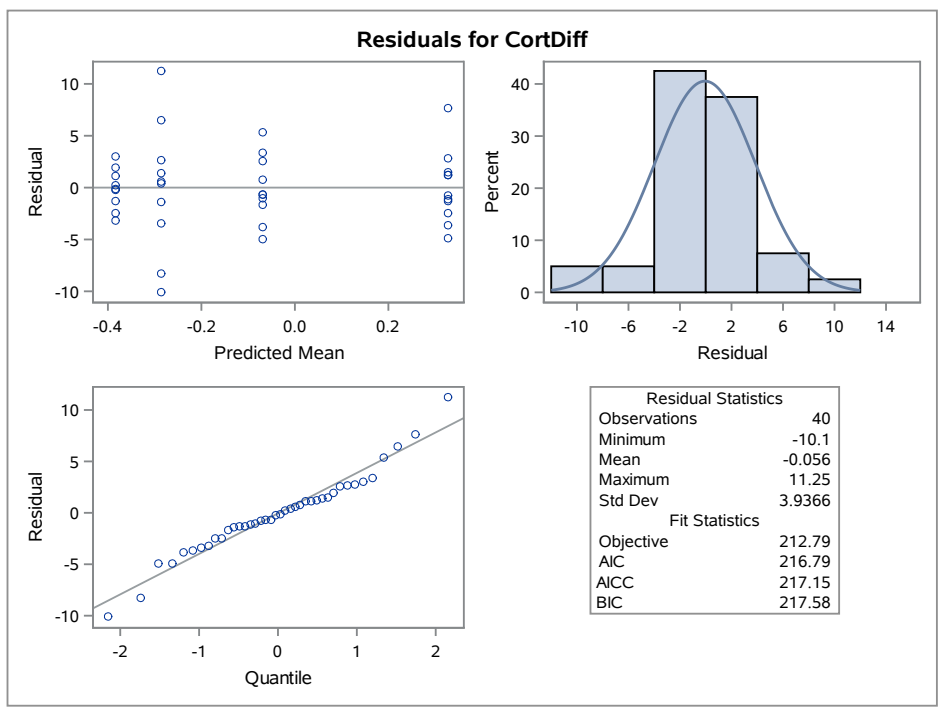


Day 1 Cortisol Post-Startle Average

The Mixed Procedure

\begin{tabular}{|l|r|r|r|r|}
\hline \multicolumn{5}{|c|}{ Type 3 Tests of Fixed Effects } \\
\hline Effect & $\begin{array}{r}\text { Num } \\
\text { DF }\end{array}$ & $\begin{array}{r}\text { Den } \\
\text { DF }\end{array}$ & F Value & Pr > F \\
\hline CortPosttestPlate & 4 & 23.5 & 2.85 & 0.0465 \\
\hline Treatment & 3 & 23.2 & 0.28 & 0.8367 \\
\hline
\end{tabular}

\begin{tabular}{|l|r|r|r|r|r|r|}
\hline \multicolumn{7}{|c|}{ Least Squares Means Estimate } \\
\hline Effect & Label & Estimate & $\begin{array}{r}\text { Standard } \\
\text { Error }\end{array}$ & DF & t Value & Pr $>$ tt \\
\hline Treatment & Row 1 & 0.4193 & 1.5072 & 23.19 & 0.28 & 0.7833 \\
\hline
\end{tabular}

Least Squares Means

\begin{tabular}{|l|l|r|r|r|r|r|}
\hline \multicolumn{7}{|c|}{ Least Squares Means } \\
\hline Effect & Treatment & Estimate & $\begin{array}{r}\text { Standard } \\
\text { Error }\end{array}$ & DF & t Value & Pr > $>$ t \\
\hline Treatment & 0 & 12.8127 & 3.1136 & 13.2 & 4.12 & 0.0012 \\
\hline Treatment & 20 & 11.9544 & 3.0832 & 12.7 & 3.88 & 0.0020 \\
\hline Treatment & 40 & 11.8746 & 3.0795 & 12.7 & 3.86 & 0.0021 \\
\hline Treatment & 60 & 13.3511 & 3.1609 & 13.9 & 4.22 & 0.0009 \\
\hline
\end{tabular}

\begin{tabular}{|l|l|l|r|r|r|r|r|}
\hline \multicolumn{7}{|c|}{ Differences of Least Squares Means } \\
\hline Effect & Treatment & Treatment & Estimate & $\begin{array}{r}\text { Standard } \\
\text { Error }\end{array}$ & DF & t Value & Pr $>$ It \\
\hline Treatment & 0 & 20 & 0.8583 & 1.8025 & 23.1 & 0.48 & 0.6384 \\
\hline Treatment & 0 & 40 & 0.9381 & 1.7996 & 23.1 & 0.52 & 0.6071 \\
\hline Treatment & 0 & 60 & -0.5384 & 1.9102 & 23.3 & -0.28 & 0.7806 \\
\hline Treatment & 20 & 40 & 0.07982 & 1.7226 & 23.1 & 0.05 & 0.9634 \\
\hline Treatment & 20 & 60 & -1.3967 & 1.8523 & 23.2 & -0.75 & 0.4584 \\
\hline Treatment & 40 & 60 & -1.4765 & 1.8881 & 23.2 & -0.78 & 0.4421 \\
\hline
\end{tabular}

Day 3 Cortisol Post-Startle Average

The Mixed Procedure

\begin{tabular}{|l|r|r|r|r|}
\hline \multicolumn{5}{|c|}{ Type 3 Tests of Fixed Effects } \\
\hline Effect & $\begin{array}{r}\text { Num } \\
\text { D F }\end{array}$ & $\begin{array}{r}\text { Den } \\
\text { DF }\end{array}$ & F Value & Pr > F \\
\hline CortPosttestPlate & 4 & 23.4 & 1.70 & 0.1835 \\
\hline Treatment & 3 & 22.9 & 0.96 & 0.4263 \\
\hline
\end{tabular}

\begin{tabular}{|l|r|r|r|r|r|r|}
\hline \multicolumn{7}{|c|}{ Least Squares Means Estimate } \\
\hline Effect & Label & Estimate & $\begin{array}{r}\text { Standard } \\
\text { Error }\end{array}$ & DF & t Value & Pr $>\mid$ tt \\
\hline Treatment & Row 1 & 1.0556 & 1.7400 & 22.87 & 0.61 & 0.5500 \\
\hline
\end{tabular}

\begin{tabular}{|l|l|r|r|r|r|l|}
\hline \multicolumn{7}{|c|}{ Least Squares Means } \\
\hline Effect & Treatment & Estimate & $\begin{array}{r}\text { Standard } \\
\text { Error }\end{array}$ & DF & t Value & Pr $>$ tt 1 \\
\hline Treatment & 0 & 12.6517 & 2.6176 & 17.2 & 4.83 & 0.0001 \\
\hline Treatment & 20 & 10.5505 & 2.5295 & 15.6 & 4.17 & 0.0008 \\
\hline Treatment & 40 & 13.5042 & 2.5504 & 16 & 5.29 & $<.0001$ \\
\hline Treatment & 60 & 10.7338 & 2.7088 & 19.1 & 3.96 & 0.0008 \\
\hline
\end{tabular}

Differences of Least Squares Means

\begin{tabular}{|l|l|l|r|r|r|r|r|}
\hline \multicolumn{7}{|c|}{ Differences of Least Squares Means } \\
\hline Effect & Treatment & Treatment & Estimate & $\begin{array}{r}\text { Standard } \\
\text { Error }\end{array}$ & DF & t Value & Pr $>$ |t| \\
\hline Treatment & 0 & 20 & 2.1013 & 2.0974 & 23 & 1.00 & 0.3269 \\
\hline Treatment & 0 & 40 & -0.8525 & 2.0856 & 22.9 & -0.41 & 0.6865 \\
\hline Treatment & 0 & 60 & 1.9180 & 2.2207 & 22.8 & 0.86 & 0.3968 \\
\hline Treatment & 20 & 40 & -2.9537 & 2.0272 & 22.7 & -1.46 & 0.1588 \\
\hline Treatment & 20 & 60 & -0.1833 & 2.2046 & 23.1 & -0.08 & 0.9345 \\
\hline Treatment & 40 & 60 & 2.7704 & 2.1962 & 23 & 1.26 & 0.2198 \\
\hline
\end{tabular}


Day 1 Cortisol Post-Startle Average

The Mixed Procedure
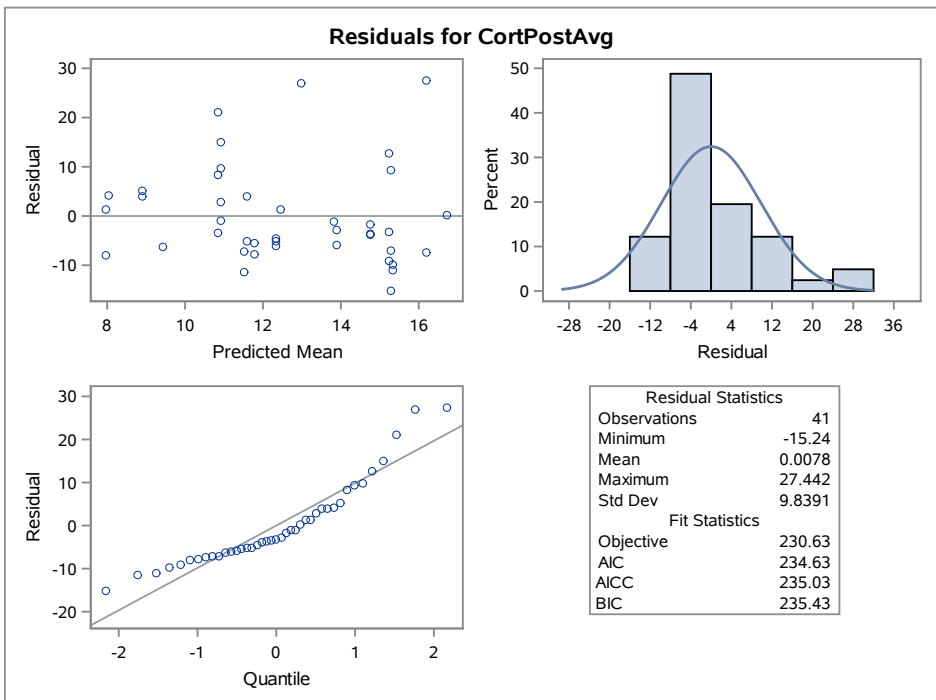

Residual Statistics
Observations
Minimum

Minimum

Mean

Maximum
Std Dev

Objectiv
AIC

AlCC

27.442
9.8391

230.63
234.63
235.03
Quantile
Day 3 Cortisol Post-Startle Average

The Mixed Procedure

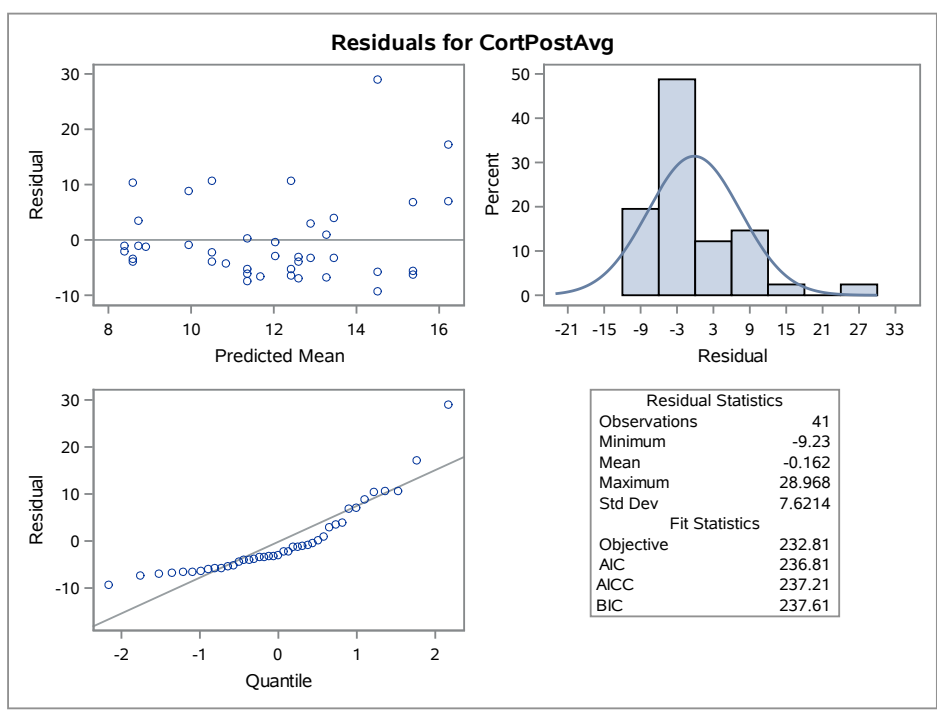


APPENDIX 5

SAS SUMMARY OUTPUT

Group 1 Amino Acid Data

The MEANS Procedure

\begin{tabular}{|c|c|c|c|c|c|c|c|c|c|}
\hline Treatment & Day & $\begin{array}{r}\mathrm{N} \\
\text { Obs }\end{array}$ & Variable & Label & $\mathbf{N}$ & Mean & Std Dev & Minimum & Maximum \\
\hline \multirow[t]{4}{*}{0} & \multirow[t]{2}{*}{1} & \multirow[t]{2}{*}{4} & TRP_Diff & TRP_Diff & 4 & $\begin{array}{r}1.7750000 \\
\end{array}$ & 1.1236103 & 0.3000000 & 2.7000000 \\
\hline & & & RatioDiff & RatioDiff & 3 & -0.000170638 & 0.0027832 & -0.0030187 & 0.0025429 \\
\hline & \multirow[t]{2}{*}{3} & \multirow[t]{2}{*}{4} & TRP_Diff & TRP_Diff & 4 & 0.6000000 & 2.3122860 & -2.1000000 & 3.5000000 \\
\hline & & & RatioDiff & RatioDiff & 3 & 0.000430384 & 0.0048699 & -0.0033927 & 0.0059132 \\
\hline \multirow[t]{4}{*}{20} & \multirow[t]{2}{*}{1} & \multirow[t]{2}{*}{4} & TRP Diff & TRP Diff & 3 & 44.4333333 & 36.9932877 & 2.0000000 & 69.9000000 \\
\hline & & & RatiōDiff & RatioDiff & 3 & 0.0872213 & 0.0716457 & 0.0051378 & 0.1371981 \\
\hline & \multirow[t]{2}{*}{3} & \multirow[t]{2}{*}{4} & TRP_Diff & TRP_Diff & 4 & 4.5000000 & 9.0225643 & -8.1000000 & 13.0000000 \\
\hline & & & RatioDiff & RatioDiff & 4 & 0.0154875 & 0.0279486 & -0.0243351 & 0.0394599 \\
\hline \multirow[t]{4}{*}{40} & \multirow[t]{2}{*}{1} & \multirow[t]{2}{*}{4} & TRP_Diff & TRP_Diff & 4 & 68.3500000 & 5.7494927 & 60.0000000 & 73.0000000 \\
\hline & & & RatioDiff & RatioDiff & 4 & 0.1357226 & 0.0112667 & 0.1193248 & 0.1448863 \\
\hline & \multirow[t]{2}{*}{3} & \multirow[t]{2}{*}{4} & TRP_Diff & TRP_Diff & 4 & -5.2000000 & 21.5364807 & -36.9000000 & 10.1000000 \\
\hline & & & RatioDiff & RatioDiff & 4 & -0.0125671 & 0.0415631 & -0.0654264 & 0.0232422 \\
\hline \multirow[t]{4}{*}{60} & \multirow[t]{2}{*}{1} & \multirow[t]{2}{*}{4} & TRP Diff & TRP Diff & 4 & 107.0750000 & 11.1622504 & 98.8000000 & 123.0000000 \\
\hline & & & Ratiōiff & Ratiōiff & 3 & 0.1955426 & 0.0068534 & 0.1879360 & 0.2012364 \\
\hline & \multirow[t]{2}{*}{3} & \multirow[t]{2}{*}{4} & TRP_Diff & TRP_Diff & 3 & 6.6000000 & 3.5552778 & 2.6000000 & 9.4000000 \\
\hline & & & RatioDiff & RatioDiff & 2 & 0.0037982 & 0.0137460 & -0.0059217 & 0.0135181 \\
\hline
\end{tabular}




\section{Difference in serum free Trp}

\section{The Mixed Procedure}

\begin{tabular}{|l|r|r|r|r|}
\hline \multicolumn{5}{|c|}{ Type 3 Tests of Fixed Effects } \\
\hline Effect & $\begin{array}{r}\text { Num } \\
\text { DF }\end{array}$ & $\begin{array}{r}\text { Den } \\
\text { DF }\end{array}$ & F Value & Pr > F \\
\hline Treatment & 3 & 22 & 17.45 & $<.0001$ \\
\hline Day & 1 & 22 & 96.09 & $<.0001$ \\
\hline Treatment*Day & 3 & 22 & 15.55 & $<.0001$ \\
\hline
\end{tabular}

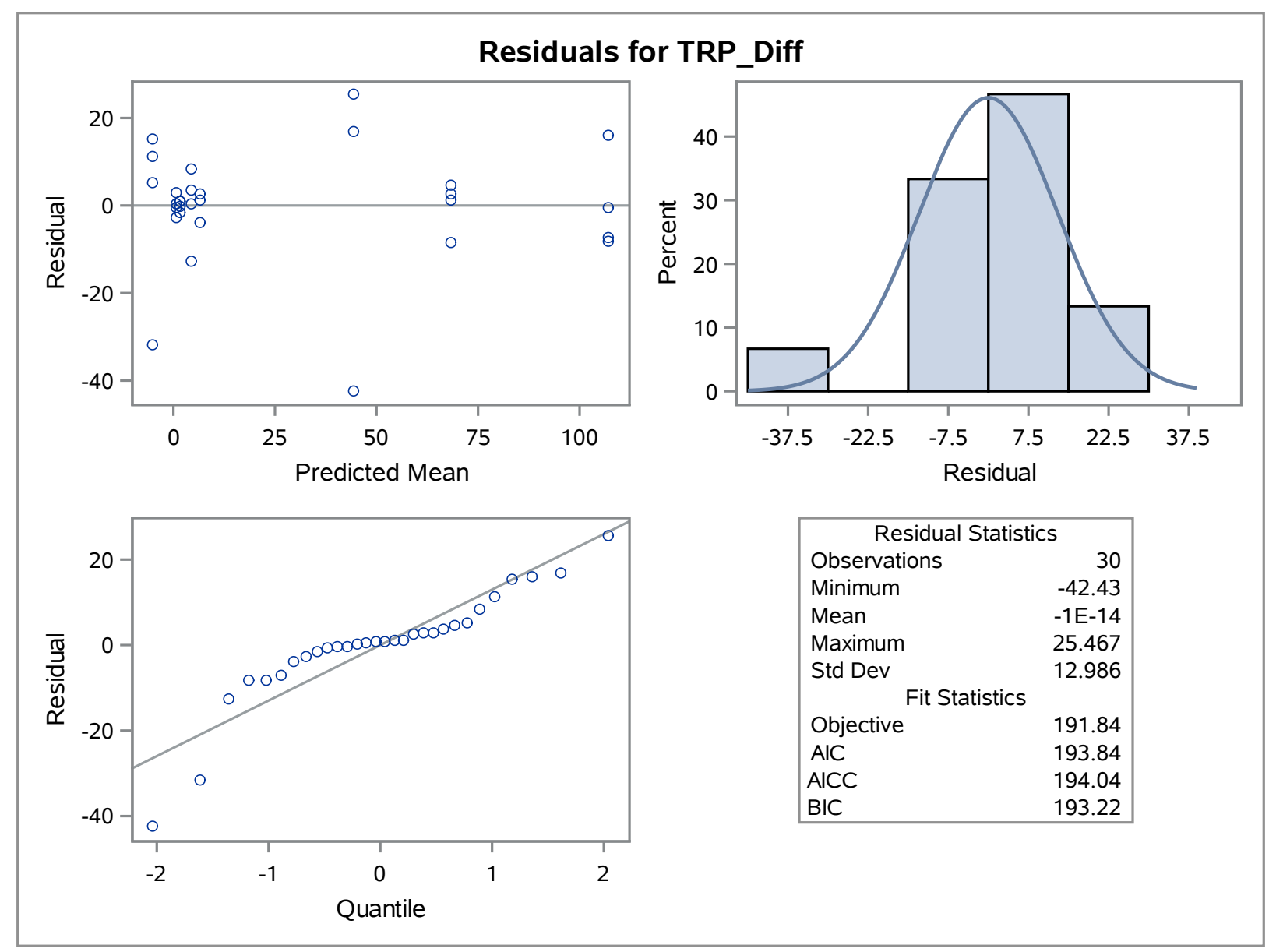




\section{DAY 1: TRP_DIFF}

\begin{tabular}{|l|r|r|r|r|}
\hline \multicolumn{5}{|c|}{ F Test for Treatment*Day Least Squares } \\
Means Slice \\
\hline Slice & $\begin{array}{r}\text { Num } \\
\text { DF }\end{array}$ & $\begin{array}{r}\text { Den } \\
\text { DF }\end{array}$ & F Value & Pr > F \\
\hline Day 1 & 3 & 22 & 34.80 & $<.0001$ \\
\hline
\end{tabular}

\begin{tabular}{|l|l|l|r|r|r|r|r|}
\hline \multicolumn{7}{|c|}{ Simple Differences of Treatment*Day Least Squares Means } \\
\hline Slice & Treatment & Treatment & Estimate & $\begin{array}{r}\text { Standard } \\
\text { Error }\end{array}$ & DF & t Value & Pr > |t| \\
\hline Day 1 & 0 & 20 & -42.6583 & 11.3877 & 22 & -3.75 & 0.0011 \\
\hline Day 1 & 0 & 40 & -66.5750 & 10.5429 & 22 & -6.31 & $<.0001$ \\
\hline Day 1 & 0 & 60 & -105.30 & 10.5429 & 22 & -9.99 & $<.0001$ \\
\hline Day 1 & 20 & 40 & -23.9167 & 11.3877 & 22 & -2.10 & 0.0474 \\
\hline Day 1 & 20 & 60 & -62.6417 & 11.3877 & 22 & -5.50 & $<.0001$ \\
\hline Day 1 & 40 & 60 & -38.7250 & 10.5429 & 22 & -3.67 & 0.0013 \\
\hline
\end{tabular}

\section{DAY 3: TRP_DIFF}

\begin{tabular}{|l|r|r|r|r|}
\hline \multicolumn{5}{|c|}{ F Test for Treatment ${ }^{\star}$ Day Least Squares } \\
Means Slice \\
\hline Slice & $\begin{array}{r}\text { Num } \\
\text { DF }\end{array}$ & $\begin{array}{r}\text { Den } \\
\text { DF }\end{array}$ & F Value & Pr > F \\
\hline Day 3 & 3 & 22 & 0.44 & 0.7239 \\
\hline
\end{tabular}

\begin{tabular}{|l|l|l|r|r|r|r|r|}
\hline \multicolumn{7}{|c|}{ Simple Differences of Treatment*Day Least Squares Means } \\
\hline Slice & Treatment & Treatment & Estimate & $\begin{array}{r}\text { Standard } \\
\text { Error }\end{array}$ & DF & t Value & Pr > |t| \\
\hline Day 3 & 0 & 20 & -3.9000 & 10.5429 & 22 & -0.37 & 0.7150 \\
\hline Day 3 & 0 & 40 & 5.8000 & 10.5429 & 22 & 0.55 & 0.5878 \\
\hline Day 3 & 0 & 60 & -6.0000 & 11.3877 & 22 & -0.53 & 0.6035 \\
\hline Day 3 & 20 & 40 & 9.7000 & 10.5429 & 22 & 0.92 & 0.3675 \\
\hline Day 3 & 20 & 60 & -2.1000 & 11.3877 & 22 & -0.18 & 0.8554 \\
\hline Day 3 & 40 & 60 & -11.8000 & 11.3877 & 22 & -1.04 & 0.3114 \\
\hline
\end{tabular}




\section{Difference in TRP:LNAA}

The Mixed Procedure

\begin{tabular}{|l|r|r|r|r|}
\hline \multicolumn{5}{|c|}{ Type 3 Tests of Fixed Effects } \\
\hline Effect & $\begin{array}{r}\text { Num } \\
\text { DF }\end{array}$ & $\begin{array}{r}\text { Den } \\
\text { DF }\end{array}$ & F Value & Pr > F \\
\hline Treatment & 3 & 18 & 9.00 & 0.0007 \\
\hline Day & 1 & 18 & 63.61 & $<.0001$ \\
\hline Treatment*Day & 3 & 18 & 10.14 & 0.0004 \\
\hline
\end{tabular}

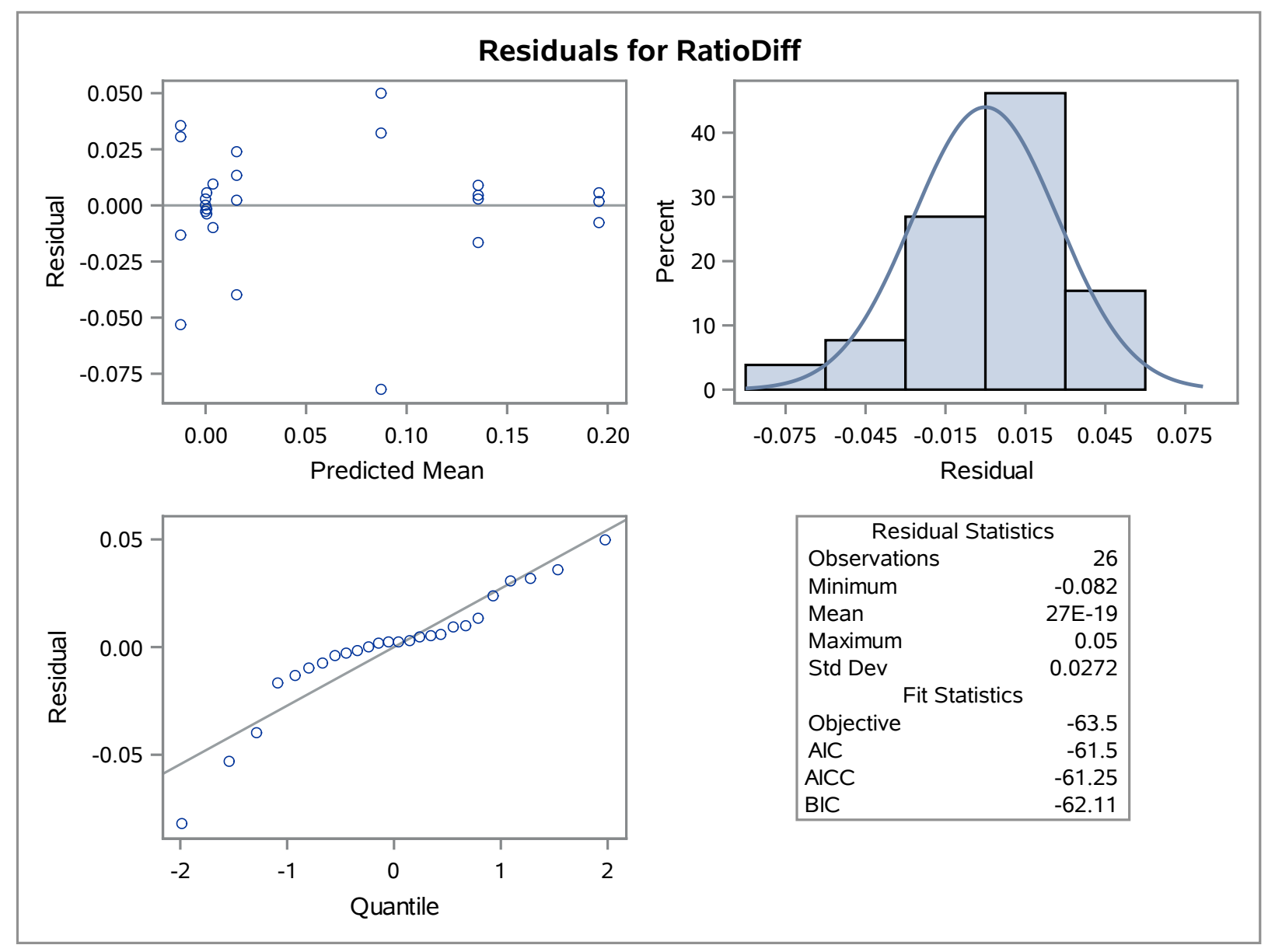




\section{DAY 1: RATIO DIFF}

\begin{tabular}{|l|r|r|r|r|}
\hline \multicolumn{5}{|c|}{ F Test for Treatment*Day Least Squares } \\
Means Slice \\
\hline Slice & $\begin{array}{r}\text { Num } \\
\text { DF }\end{array}$ & $\begin{array}{r}\text { Den } \\
\text { DF }\end{array}$ & F Value & Pr $>$ F \\
\hline Day 1 & 3 & 18 & 20.23 & $<.0001$ \\
\hline
\end{tabular}

\begin{tabular}{|l|l|l|r|r|r|r|r|}
\hline \multicolumn{7}{|c|}{ Simple Differences of Treatment*Day Least Squares Means } \\
\hline Slice & Treatment & Treatment & Estimate & $\begin{array}{r}\text { Standard } \\
\text { Error }\end{array}$ & DF & t Value & Pr > |t| \\
\hline Day 1 & 0 & 20 & -0.08739 & 0.02619 & 18 & -3.34 & 0.0037 \\
\hline Day 1 & 0 & 40 & -0.1359 & 0.02450 & 18 & -5.55 & $<.0001$ \\
\hline Day 1 & 0 & 60 & -0.1957 & 0.02619 & 18 & -7.47 & $<.0001$ \\
\hline Day 1 & 20 & 40 & -0.04850 & 0.02450 & 18 & -1.98 & 0.0632 \\
\hline Day 1 & 20 & 60 & -0.1083 & 0.02619 & 18 & -4.14 & 0.0006 \\
\hline Day 1 & 40 & 60 & -0.05982 & 0.02450 & 18 & -2.44 & 0.0252 \\
\hline
\end{tabular}

\section{DAY 3: RATIO DIFF}

\begin{tabular}{|l|r|r|r|r|}
\hline \multicolumn{5}{|c|}{ F Test for Treatment*Day Least Squares } \\
Means Slice \\
\hline Slice & $\begin{array}{r}\text { Num } \\
\text { DF }\end{array}$ & $\begin{array}{r}\text { Den } \\
\text { DF }\end{array}$ & F Value & Pr > F \\
\hline Day 3 & 3 & 18 & 0.51 & 0.6775 \\
\hline
\end{tabular}

\begin{tabular}{|l|l|l|r|r|r|r|r|}
\hline \multicolumn{7}{|c|}{ Simple Differences of Treatment*Day Least Squares Means } \\
\hline Slice & Treatment & Treatment & Estimate & $\begin{array}{r}\text { Standard } \\
\text { Error }\end{array}$ & DF & t Value & Pr > |t| \\
\hline Day 3 & 0 & 20 & -0.01506 & 0.02450 & 18 & -0.61 & 0.5465 \\
\hline Day 3 & 0 & 40 & 0.01300 & 0.02450 & 18 & 0.53 & 0.6022 \\
\hline Day 3 & 0 & 60 & -0.00337 & 0.02928 & 18 & -0.12 & 0.9097 \\
\hline Day 3 & 20 & 40 & 0.02805 & 0.02268 & 18 & 1.24 & 0.2320 \\
\hline Day 3 & 20 & 60 & 0.01169 & 0.02778 & 18 & 0.42 & 0.6789 \\
\hline Day 3 & 40 & 60 & -0.01637 & 0.02778 & 18 & -0.59 & 0.5631 \\
\hline
\end{tabular}




\section{Comparing treatment numbers \\ Washout serum free Trp}

The Mixed Procedure

\begin{tabular}{|l|l|l|r|r|r|r|r|}
\hline \multicolumn{7}{|c|}{ Differences of Least Squares Means } \\
\hline Effect & TrtNum & TrtNum & Estimate & $\begin{array}{r}\text { Standard } \\
\text { Error }\end{array}$ & DF & t Value & Pr > |t| \\
\hline TrtNum & 1 & 2 & -0.4500 & 2.3466 & 12 & -0.19 & 0.8511 \\
\hline TrtNum & 1 & 3 & 0.8500 & 2.3466 & 12 & 0.36 & 0.7235 \\
\hline TrtNum & 1 & 4 & 2.8000 & 2.3466 & 12 & 1.19 & 0.2558 \\
\hline TrtNum & 2 & 3 & 1.3000 & 2.3466 & 12 & 0.55 & 0.5898 \\
\hline TrtNum & 2 & 4 & 3.2500 & 2.3466 & 12 & 1.38 & 0.1913 \\
\hline TrtNum & 3 & 4 & 1.9500 & 2.3466 & 12 & 0.83 & 0.4222 \\
\hline
\end{tabular}

\section{Comparing treatment numbers \\ Washout serum TRP:LNAA}

\section{The Mixed Procedure}

\begin{tabular}{|l|l|l|r|r|r|r|r|}
\hline \multicolumn{7}{|c|}{ Differences of Least Squares Means } \\
\hline Effect & TrtNum & TrtNum & Estimate & $\begin{array}{r}\text { Standard } \\
\text { Error }\end{array}$ & DF & t Value & Pr > |t| \\
\hline TrtNum & 1 & 2 & -0.00119 & 0.004447 & 12 & -0.27 & 0.7938 \\
\hline TrtNum & 1 & 3 & 0.002036 & 0.004447 & 12 & 0.46 & 0.6552 \\
\hline TrtNum & 1 & 4 & 0.006502 & 0.004447 & 12 & 1.46 & 0.1694 \\
\hline TrtNum & 2 & 3 & 0.003225 & 0.004447 & 12 & 0.73 & 0.4823 \\
\hline TrtNum & 2 & 4 & 0.007690 & 0.004447 & 12 & 1.73 & 0.1094 \\
\hline TrtNum & 3 & 4 & 0.004466 & 0.004447 & 12 & 1.00 & 0.3351 \\
\hline
\end{tabular}




\section{Comparisons between days and blood collections serum free Trp}

The Mixed Procedure

\begin{tabular}{|l|l|l|r|r|r|r|r|}
\hline \multicolumn{7}{|c|}{ Differences of Least Squares Means } \\
\hline Effect & DBC & DBC & Estimate & $\begin{array}{r}\text { Standard } \\
\text { Error }\end{array}$ & DF & t Value & Pr > |t| \\
\hline DBC & 11 & 12 & -56.2678 & 7.7451 & 43.2 & -7.26 & $<.0001$ \\
\hline DBC & 11 & 31 & -56.9563 & 7.5829 & 43.1 & -7.51 & $<.0001$ \\
\hline DBC & 11 & 32 & -57.1518 & 7.7451 & 43.2 & -7.38 & $<.0001$ \\
\hline DBC & 12 & 31 & -0.6884 & 7.7451 & 43.2 & -0.09 & 0.9296 \\
\hline DBC & 12 & 32 & -0.8840 & 7.9101 & 43.4 & -0.11 & 0.9115 \\
\hline DBC & 31 & 32 & -0.1956 & 7.7451 & 43.2 & -0.03 & 0.9800 \\
\hline
\end{tabular}

$\mathrm{DBC}=\mathrm{DayBC}$

Comparisons between days and blood collections serum TRP:LNAA

The Mixed Procedure

\begin{tabular}{|l|l|l|r|r|r|r|r|}
\hline \multicolumn{7}{|c|}{ Differences of Least Squares Means } \\
\hline Effect & DBC & DBC & Estimate & $\begin{array}{r}\text { Standard } \\
\text { Error }\end{array}$ & DF & $t$ Value & Pr > |t| \\
\hline DBC & 11 & 12 & -0.1069 & 0.01576 & 40 & -6.78 & $<.0001$ \\
\hline DBC & 11 & 31 & -0.1094 & 0.01465 & 39.2 & -7.47 & $<.0001$ \\
\hline DBC & 11 & 32 & -0.1091 & 0.01576 & 40 & -6.93 & $<.0001$ \\
\hline DBC & 12 & 31 & -0.00255 & 0.01576 & 40 & -0.16 & 0.8720 \\
\hline DBC & 12 & 32 & -0.00227 & 0.01644 & 39.6 & -0.14 & 0.8911 \\
\hline DBC & 31 & 32 & 0.000289 & 0.01576 & 40 & 0.02 & 0.9855 \\
\hline
\end{tabular}

DBC $=$ DayBC 